YITP-SB-13-22

\title{
SOV approach for integrable quantum models associated to general representations on spin-1/2 chains of the 8-vertex reflection algebra
}

\author{
S. Faldella1, G. Niccoli2
}

\begin{abstract}
The analysis of the transfer matrices associated to the most general representations of the 8-vertex reflection algebra on spin- $1 / 2$ chains is here implemented by introducing a quantum separation of variables (SOV) method which generalizes to these integrable quantum models the method first introduced by Sklyanin. More in detail, for the representations reproducing in their homogeneous limits the open XYZ spin- $1 / 2$ quantum chains with the most general integrable boundary conditions, we explicitly construct representations of the 8-vertex reflection algebras for which the transfer matrix spectral problem is separated. Then, in these SOV representations we get the complete characterization of the transfer matrix spectrum (eigenvalues and eigenstates) and its non-degeneracy. Moreover, we present the first fundamental step toward the characterization of the dynamics of these models by deriving determinant formulae for the matrix elements of the identity on separated states, which apply in particular to transfer matrix eigenstates. The comparison of our analysis for the 8-vertex reflection algebra with that of [1, 2] for the 6-vertex one leads to the interesting remark that a profound similarity in both the characterization of the spectral problems and of the scalar products exists for these two different realizations of the reflection algebra once they are described by SOV method. As it will be shown in a future publication, this remarkable similarity will be at the basis of the simultaneous determination of form factors of local operators of integrable quantum models associated to general reflection algebra representations of both 8-vertex and 6-vertex type.
\end{abstract}

\footnotetext{
${ }^{1}$ IMB, UMR 5584 du CNRS, Université de Bourgogne, France, Simone.Faldella@u-bourgogne.fr

${ }^{2}$ YITP, Stony Brook University, New York 11794-3840, USA, niccoli@ max2.physics.sunysb.edu
} 


\section{Contents}

1 Introduction

2 Reflection algebra

2.1 Representations of 8 -vertex reflection algebra on spin-1/2 chains . . . . . . . . . 4

2.2 Properties of reflection algebra generators $\ldots \ldots \ldots \ldots \ldots$

3 Baxter's gauge transformations and central properties 9

3.1 Notations . . . . . . . . . . . . . . . . . . . . . . . 9

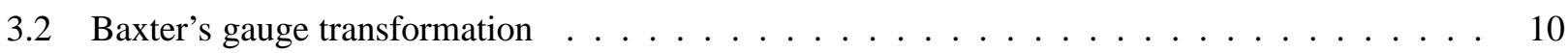

3.3 Gauge transformed boundary operators and their properties . . . . . . . . . . . 11

3.3 .1 Definitions . . . . . . . . . . . . . . . . . . . . 11

3.3.2 Main symmetries . . . . . . . . . . . . . . . . . . 11

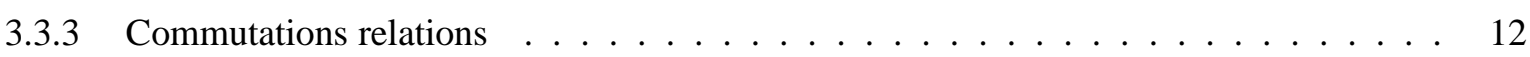

$3.3 .4 \quad \beta$-parity relations . . . . . . . . . . . . . . . 13

3.4 Transfer matrix representations in terms of gauge transformed boundary operators . . . . . . 14

4 SOV representations $\quad 17$

4.1 Gauge transformed reflection algebra in $\mathcal{B}_{-}(\mid \beta)-$ SOV representations $\ldots \ldots \ldots$

4.1.1 Simultaneous $B(\lambda \mid \beta)$ and $\bar{B}(\lambda \mid \beta)$ bulk left reference state $\ldots \ldots \ldots \ldots$

4.1.2 Simultaneous $C(\lambda \mid \beta)$ and $\bar{C}(\lambda \mid \beta)$ bulk right reference state $\ldots \ldots \ldots \ldots$

4.1.3 Gauge transformed reflection algebra in left $\mathcal{B}_{-}(\mid \beta)-$ SOV representations $\ldots \ldots \ldots$

4.1.4 Gauge transformed reflection algebra in right $\mathcal{B}_{-}(\mid \beta)$-SOV representations . . . . . 22

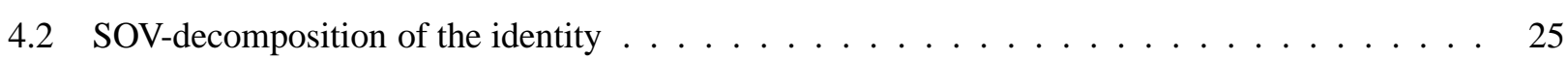

5 Separate variable characterization of transfer matrix spectrum 27

6 Scalar Products

7 Conclusion and outlook

References 


\section{Introduction}

In the framework of the quantum inverse scattering method (QISM) [3]-[15], we analyze the class of 1D lattice integrable quantum models associated to monodromy matrices which are the most general solutions of the reflection algebra [16]-[21] w.r.t. the elliptic 8-vertex R-matrix. It is worth commenting that these models have attracted a large interest which goes beyond the community of quantum integrability. This is in particular true for representations associated to non-diagonal integrable boundary matrices which have proven to be hard to describe by standard Bethe ansatz analysis [22]-[29] and which allow to describe interesting out of equilibrium physical systems and for which already in the 6-vertex case a very large literature has been developed to address with different methods the associated transfer matrix spectral problem 1 [30]-[46]. The homogeneous limit of the 8-vertex reflection algebra representations that we analyze in this paper leads to the description of open XYZ spin-1/2 quantum chains with the most general integrable boundary conditions. For these integrable quantum models, we introduce a quantum version of the separation of variables (SOV) in the spirit of the works [47] pioneered by Sklyanin. In our SOV approach we both obtain the complete characterization of the transfer matrix spectrum (eigenvalues and eigenstates) and we derive simple determinant formulae for the scalar products of transfer matrix eigenstates. In particular, starting from the original spin-1/2 representations of the 8 -vertex reflection algebra we explicitly construct a new (SOV) basis of the space of the representations for which the transfer matrix spectral problem is separated and completely characterized in terms of the set of solutions to a inhomogeneous system of $\mathrm{N}$ quadratic equations in $\mathrm{N}$ unknowns, where $\mathrm{N}$ is the size of the chain. It is also worth remarking that in our SOV approach, it is simple to prove the complete integrability 2 of the associated quantum models, i.e. the fact that the transfer matrix forms a complete set of commuting conserved charges on the space of the representation. One fundamental finding of the SOV analysis here developed is that the pseudo-measure entering in the SOV spectral decomposition of the identity is simply expressed as the inverse of determinants of $\mathrm{N} \times \mathrm{N}$ Vandermonde's matrices. It is then central to observe that all the SOV representations constructed so far in [51]-[53] associated to 6-vertex representations of the Yang-Baxter and reflection algebra share just the same structure of inverse of Vandermonde's determinant for these pseudomeasures. This observation is even more important once we point out that the SOV spectral decompositions of the identity have a different structure of the pseudo-measures in the case of the transfer matrices associated to both the 8-vertex representation of the Yang-Baxter algebra [57] and the elliptic representations of the 6-vertex dynamical Yang-Baxter algebra [58]. Indeed, in [56] and [59] a different determinant form has been derived for these pseudomeasures. It is then the combined use of the SOV method and of the reflection algebra which allows an amazing simultaneous description of the spectral and dynamical problems for the 8-vertex and 6-vertex transfer matrices which will be used in future publications to solve simultaneously these dynamical problems.

Let us resume here some results and difficulties appearing in the preexisting literature on the analysis of these 8-vertex integrable quantum models; this also to clarify the reasons of interest and novelty in our SOV analysis. In [57] Baxter has defined the intertwining vectors or gauge transformations, in order to be able to use Bethe ansatz techniques to analyze the spectral problem 3 of the transfer matrix associated to 8-vertex Yang-Baxter algebra representations. The use of gauge transformations allows in particular to define pseudo-reference states opening the possibility to analyze these 8 -vertex spectral problems by using the algebraic Bethe ansatz (ABA) [3]-[4] as derived in [5]. The Baxter's gauge transformations have been used also in [64] to analyze the spectral problem associated to the 8-vertex reflection algebra in the ABA framework, see also [30] for the 6-vertex case. Anyhow, it is important to

\footnotetext{
${ }^{1}$ See the papers [1, 2] for a discussion of this point and for more details on the role of the cited references.

${ }^{2}$ This definition can be seen as the natural quantum analogous of the classical Liouville complete integrability and it was shown in the SOV framework for a series of other integrable quantum models [48]-[56].

${ }^{3}$ Under periodic boundary conditions the spectral problems of these transfer matrices have been analyzed also by the Baxter's Q-operator techniques, see [57, 60] and also the series of papers [61]-[63].
} 
remark that in the framework of Bethe ansatz persists the general problem related to the proof of the completeness of the spectrum description 4 and constrains are required to implement the spectral analysis of these models. In the case of 8-vertex transfer matrices associated to periodic boundary conditions the following two constrains are introduced: the number of sites of the quantum chains has to be even and the values allowed of the coupling constant $\eta$ are restricted to the elliptic roots of unit. These two constrains do not appear instead in the description by ABA of the 8-vertex transfer matrices associated to open boundary conditions, which already in this ABA framework reflects a simplification occurring when we consider 8-vertex reflection algebras. However, to make ABA working it is required to introduce constrains between the boundary parameters, as done in [64], i.e. the 8-vertex transfer matrix spectral problems associated to the most general representations of the reflection algebra cannot be analyzed by using ABA. As mentioned above all these problems are overcome in our SOV framework and it is possible to describe the complete 8-vertex spectrum for all closed [56, 59] and open integrable boundary conditions. A part the constrains for the spectral analysis one central difficulty in the ABA framework is the solution of the dynamical problem. Indeed, in this 8-vertex framework a scalar product analogue 5 to the 6-vertex Slavnov's formula [70] is missing for both closed and open boundary conditions. In fact, this is the first fundamental missing step toward the computation of correlation functions according to the Lyon group method developed in [71]-[75] for the 6-vertex transfer matrix associated to the Yang-Baxter algebra representations and generalized to some classes of 6-vertex reflection algebra in [76]-[78]. It is then clear the need to overcome these problems in order to compute matrix elements of local operators on 8-vertex transfer matrix eigenstates and so the importance of the results derived in the SOV approach both here for the reflection algebra case and in [56, 59] for the Yang-Baxter algebra case.

\section{Reflection algebra}

In the framework of the quantum inverse scattering method, a class of quantum integrable models characterized by monodromy matrices solutions of the 8-vertex elliptic reflection equations is here introduced.

\subsection{Representations of 8-vertex reflection algebra on spin-1/2 chains}

Let us start introducing the following $2 \times 2$ matrix [79]:

$$
\begin{aligned}
K(\lambda ; \zeta, \kappa, \tau) \equiv \frac{h(\lambda ; \zeta)}{\operatorname{sn} \tilde{\zeta}}\left(\begin{array}{cc}
\operatorname{sn}(\tilde{\lambda}+\tilde{\zeta}) & \kappa e^{\tau} \operatorname{sn} 2 \tilde{\lambda} \frac{1-k e^{-2 \tau} \operatorname{sn}^{2} \tilde{\lambda}}{1-k^{2} \operatorname{sn}^{2} \tilde{\zeta} \operatorname{sn}^{2} \tilde{\lambda}} \\
\kappa e^{-\tau} \operatorname{sn} 2 \tilde{\lambda} \frac{1-k e^{2 \tau} \operatorname{sn}^{2} \tilde{\lambda}}{1-k^{2} \operatorname{sn}^{2} \tilde{\lambda} \operatorname{sn}^{2} \tilde{\zeta}} & \operatorname{sn}(\tilde{\zeta}-\tilde{\lambda})
\end{array}\right) \\
=\left(\begin{array}{cc}
\frac{\theta_{4}(\zeta \mid 2 \omega) \theta_{4}(-\lambda+\zeta \mid 2 \omega) \theta_{1}(\lambda+\zeta \mid 2 \omega)}{\theta_{1}(\zeta \mid 2 \omega)} & \frac{\kappa e^{\tau} \theta_{1}(2 \lambda \mid 2 \omega)\left(\theta_{4}^{2}(\lambda \mid 2 \omega)-e^{-2 \tau} \theta_{1}^{2}(\lambda \mid 2 \omega)\right)}{\theta_{1}(\zeta \mid 2 \omega) \theta_{4}^{-3}(\zeta \mid 2 \omega) \theta_{4}^{2}(0 \mid 2 \omega) \theta_{1}(2 \lambda \mid 2 \omega)} \\
\frac{\kappa e^{-\tau} \theta_{1}(2 \lambda \mid 2 \omega)\left(\theta_{4}^{2}(\lambda \mid 2 \omega)-e^{2 \tau} \theta_{1}^{2}(\lambda \mid 2 \omega)\right)}{\theta_{1}(\zeta \mid 2 \omega) \theta_{4}^{-3}(\zeta \mid 2 \omega) \theta_{4}^{2}(0 \mid 2 \omega) \theta_{4}(2 \lambda \mid 2 \omega)} & \frac{\theta_{4}(\zeta \mid 2 \omega) \theta_{1}(-\lambda+\zeta \mid 2 \omega) \theta_{4}(\lambda+\zeta \mid 2 \omega)}{\theta_{1}(\zeta \mid 2 \omega)}
\end{array}\right)
\end{aligned}
$$

where 6 :

$$
h(\lambda ; \zeta) \equiv \theta_{4}(\lambda+\zeta \mid 2 \omega) \theta_{4}(\lambda-\zeta \mid 2 \omega), \quad \tilde{\lambda} \equiv 2 \mathrm{~K}_{k} \lambda, \quad \tilde{\eta} \equiv 2 \mathrm{~K}_{k} \eta, \quad \tilde{\zeta} \equiv 2 \mathrm{~K}_{k} \zeta
$$

\footnotetext{
${ }^{4}$ The numerical analysis developed in [65] provides some evidence of the completeness of the spectrum description for the periodic 8 -vertex transfer matrix.

${ }^{5}$ When some special type of double constrains on the boundary parameters are satisfied some steps in this direction have been done for both 6-vertex and 8-vertex case in [66, 67] and some related analysis appear also in [68, 69].

${ }^{6}$ The theta functions here used are those defined in [80] with the following change of notation in their arguments $(\lambda \mid x)$ instead of $(u \mid \tau)$.
} 
and:

$$
\begin{aligned}
\operatorname{sn} \tilde{\lambda} & \equiv \frac{1}{\sqrt{k}} \frac{\theta_{1}(\lambda \mid 2 \omega)}{\theta_{4}(\lambda \mid 2 \omega)}, \operatorname{cn} \tilde{\lambda} \equiv \sqrt{\frac{k^{\prime}}{k}} \frac{\theta_{2}(\lambda \mid 2 \omega)}{\theta_{4}(\lambda \mid 2 \omega)}, \operatorname{dn} \tilde{\lambda} \equiv \sqrt{k^{\prime}} \frac{\theta_{3}(\lambda \mid 2 \omega)}{\theta_{4}(\lambda \mid 2 \omega)}, \\
k & \equiv \frac{\theta_{2}^{2}(0 \mid 2 \omega)}{\theta_{3}^{2}(0 \mid 2 \omega)}, \quad k^{\prime} \equiv \frac{\theta_{4}^{2}(0 \mid 2 \omega)}{\theta_{3}^{2}(0 \mid 2 \omega)}, \quad k^{2}+k^{\prime 2}=1, \quad \mathrm{~K}_{k} \equiv \frac{\theta_{3}^{2}(0 \mid 2 \omega)}{2} .
\end{aligned}
$$

Here $\zeta, \kappa$ and $\tau$ are arbitrary complex parameters and $K(\lambda ; \zeta, \kappa, \tau)$ is the most general scalar solution 7 of the following 8 -vertex reflection equation:

$$
R_{12}^{(8 \mathrm{~V})}(\lambda-\mu) K_{1}(\lambda) R_{21}^{(8 \mathrm{~V})}(\lambda+\mu) K_{2}(\mu)=K_{2}(\mu) R_{12}^{(8 \mathrm{~V})}(\lambda+\mu) K_{1}(\lambda) R_{21}^{(8 \mathrm{~V})}(\lambda-\mu),
$$

where:

$$
R_{0 a}^{(8 \mathrm{~V})}(\lambda)=\left(\begin{array}{cccc}
\mathrm{a}(\lambda) & 0 & 0 & \mathrm{~d}(\lambda) \\
0 & \mathrm{~b}(\lambda) & \mathrm{c}(\lambda) & 0 \\
0 & \mathrm{c}(\lambda) & \mathrm{b}(\lambda) & 0 \\
\mathrm{~d}(\lambda) & 0 & 0 & \mathrm{a}(\lambda)
\end{array}\right) \in \operatorname{End}\left(\mathrm{R}_{1} \otimes \mathrm{R}_{2}\right)
$$

is the elliptic solution of the 8-vertex Yang-Baxter equation:

$$
R_{12}^{(8 \mathrm{~V})}\left(\lambda_{12}\right) R_{1 a}^{(8 \mathrm{~V})}\left(\lambda_{1}\right) R_{2 a}^{(8 \mathrm{~V})}\left(\lambda_{2}\right)=R_{2 a}^{(8 \mathrm{~V})}\left(\lambda_{2}\right) R_{1 a}^{(8 \mathrm{~V})}\left(\lambda_{1}\right) R_{12}^{(8 \mathrm{~V})}\left(\lambda_{12}\right),
$$

$\mathrm{R}_{x} \simeq \mathbb{C}^{2}$ is a 2-dimensional linear space and:

$$
\begin{array}{ll}
\mathrm{a}(\lambda) \equiv \frac{2 \theta_{4}(\eta \mid 2 \omega) \theta_{1}(\lambda+\eta \mid 2 \omega) \theta_{4}(\lambda \mid 2 \omega)}{\theta_{2}(0 \mid \omega) \theta_{4}(0 \mid 2 \omega)}, & \mathrm{b}(\lambda) \equiv \frac{2 \theta_{4}(\eta \mid 2 \omega) \theta_{1}(\lambda \mid 2 \omega) \theta_{4}(\lambda+\eta \mid 2 \omega)}{\theta_{2}(0 \mid \omega) \theta_{4}(0 \mid 2 \omega)}, \\
\mathrm{c}(\lambda) \equiv \frac{2 \theta_{1}(\eta \mid 2 \omega) \theta_{4}(\lambda \mid 2 \omega) \theta_{4}(\lambda+\eta \mid 2 \omega)}{\theta_{2}(0 \mid \omega) \theta_{4}(0 \mid 2 \omega)}, & \mathrm{d}(\lambda) \equiv \frac{2 \theta_{1}(\eta \mid 2 \omega) \theta_{1}(\lambda+\eta \mid 2 \omega) \theta_{1}(\lambda \mid 2 \omega)}{\theta_{2}(0 \mid \omega) \theta_{4}(0 \mid 2 \omega)} .
\end{array}
$$

Once we define:

$$
f(\lambda) \equiv \frac{2 \sqrt{k} \theta_{4}(\eta \mid 2 \omega) \theta_{4}(\lambda \mid 2 \omega) \theta_{4}(\lambda+\eta \mid 2 \omega)}{\theta_{2}(0 \mid \omega) \theta_{4}(0 \mid 2 \omega)},
$$

the coefficients of $R_{0 a}^{(8 \mathrm{~V})}(\lambda)$ also read:

$$
\begin{aligned}
& \mathrm{a}(\lambda)=f(\lambda) \overline{\mathrm{a}}(\tilde{\lambda}), \quad \mathrm{b}(\lambda)=f(\lambda) \overline{\mathrm{b}}(\tilde{\lambda}), \quad \mathrm{c}(\lambda)=f(\lambda) \overline{\mathrm{c}}(\tilde{\lambda}), \quad \mathrm{d}(\lambda)=f(\lambda) \overline{\mathrm{d}}(\tilde{\lambda}), \\
& \overline{\mathrm{a}}(\mu) \equiv \operatorname{sn}(\mu+\tilde{\eta}), \quad \overline{\mathrm{b}}(\mu) \equiv \operatorname{sn} \mu, \quad \overline{\mathrm{c}}(\mu) \equiv \operatorname{sn} \tilde{\eta}, \quad \overline{\mathrm{d}}(\mu) \equiv k \operatorname{sn}(\mu+\tilde{\eta}) \operatorname{sn} \mu \operatorname{sn} \tilde{\eta}
\end{aligned}
$$

Two classes of solutions to the reflection equation (2.6) are here constructed following [18] on the $2^{\mathrm{N}}$-dimensional representation space $\mathcal{R}_{\mathrm{N}} \equiv \otimes_{n=1}^{\mathrm{N}} \mathrm{R}_{n}$ of the chain. Here $\mathrm{R}_{n}$ is the 2-dimensional local space associated to the site $n$ of the chain. Let us use introduce the notations:

$$
K_{ \pm}(\lambda) \equiv K\left(\lambda \pm \eta / 2 ; \zeta_{ \pm}, \kappa_{ \pm}, \tau_{ \pm}\right)=\left(\begin{array}{cc}
a_{ \pm}(\lambda) & b_{ \pm}(\lambda) \\
c_{ \pm}(\lambda) & d_{ \pm}(\lambda)
\end{array}\right)
$$

where $\zeta_{ \pm}, \kappa_{ \pm}, \tau_{ \pm}$are arbitrary complex parameters, the $a_{ \pm}(\lambda), b_{ \pm}(\lambda), c_{ \pm}(\lambda)$ and $d_{ \pm}(\lambda)$ are defined by (2.1). The bulk monodromy matrix:

$$
\begin{aligned}
& M_{0}(\lambda)=\left(\begin{array}{cc}
A(\lambda) & B(\lambda) \\
C(\lambda) & D(\lambda)
\end{array}\right) \in \operatorname{End}\left(\mathrm{R}_{0} \otimes \mathcal{R}_{\mathrm{N}}\right), \\
& M_{0}(\lambda)=R_{0 \mathrm{~N}}^{(8 \mathrm{~V})}\left(\lambda-\xi_{\mathrm{N}}-\eta / 2\right) \ldots R_{02}^{(8 \mathrm{~V})}\left(\lambda-\xi_{2}-\eta / 2\right) R_{01}^{(8 \mathrm{~V})}\left(\lambda-\xi_{1}-\eta / 2\right),
\end{aligned}
$$

\footnotetext{
${ }^{7}$ This analysis both in the 6-vertex and in the 8-vertex case has been first developed in [81] where however only the most general solution for the 6-vertex case was found while the most general solution for the 8-vertex case was found in [79].
} 
is solution of the 8-vertex Yang-Baxter equation:

$$
R_{12}^{(8 \mathrm{~V})}(\lambda-\mu) M_{1}(\lambda) M_{2}(\mu)=M_{2}(\mu) M_{1}(\lambda) R_{12}^{(8 \mathrm{~V})}(\lambda-\mu) .
$$

Then we define the boundary monodromy matrices $\mathcal{U}_{ \pm}(\lambda) \in \operatorname{End}\left(\mathrm{R}_{0} \otimes \mathcal{R}_{\mathrm{N}}\right)$ as it follows:

$$
\begin{aligned}
\mathcal{U}_{-}(\lambda) & \equiv\left(\begin{array}{ll}
\mathcal{A}_{-}(\lambda) & \mathcal{B}_{-}(\lambda) \\
\mathcal{C}_{-}(\lambda) & \mathcal{D}_{-}(\lambda)
\end{array}\right) \equiv M_{0}(\lambda) K_{-}(\lambda) \hat{M}_{0}(\lambda) \\
\mathcal{U}_{+}^{t_{0}}(\lambda) & \equiv\left(\begin{array}{ll}
\mathcal{A}_{+}(\lambda) & \mathcal{C}_{+}(\lambda) \\
\mathcal{B}_{+}(\lambda) & \mathcal{D}_{+}(\lambda)
\end{array}\right) \equiv\left[M_{0}(\lambda)\right]^{t_{0}}\left[K_{+}(\lambda)\right]^{t_{0}}\left[\hat{M}_{0}(\lambda)\right]^{t_{0}}
\end{aligned}
$$

where:

$$
\hat{M}(\lambda)=(-1)^{\mathrm{N}} \sigma_{0}^{y}[M(-\lambda)]^{t_{0}} \sigma_{0}^{y} .
$$

$\mathcal{U}_{-}(\lambda)$ and $\mathcal{V}_{+}(\lambda) \equiv \mathcal{U}_{+}^{t_{0}}(-\lambda)$ are the two solutions of the 8-vertex reflection equation:

$$
R_{12}^{(8 \mathrm{~V})}(\lambda-\mu) \mathcal{U}_{-}^{(1)}(\lambda) R_{21}^{(8 \mathrm{~V})}(\lambda+\mu-\eta) \mathcal{U}_{-}^{(2)}(\mu)=\mathcal{U}_{-}^{(2)}(\mu) R_{12}^{(8 \mathrm{~V})}(\lambda+\mu-\eta) \mathcal{U}_{-}^{(1)}(\lambda) R_{21}^{(8 \mathrm{~V})}(\lambda-\mu)
$$

As proven in [18], from these monodromy matrices a commuting family of transfer matrices $\mathcal{T}(\lambda) \in \operatorname{End}\left(\mathcal{R}_{\mathrm{N}}\right)$ is defined by:

$$
\mathcal{T}(\lambda) \equiv \operatorname{tr}_{0}\left\{K_{+}(\lambda) M(\lambda) K_{-}(\lambda) \hat{M}(\lambda)\right\}=\operatorname{tr}_{0}\left\{K_{+}(\lambda) \mathcal{U}_{-}(\lambda)\right\}=\operatorname{tr}_{0}\left\{K_{-}(\lambda) \mathcal{U}_{+}(\lambda)\right\}
$$

We characterize here the eigenvalues and eigenstates of this transfer matrix and the matrix elements of the identity in the transfer matrix eigenstates. Note that after the homogeneous limit $\left(\xi_{n} \rightarrow 0\right.$ for any $\left.n \in\{1, \ldots, \mathrm{N}\}\right)$ the analysis here develop applies to open spin-1/2 XYZ quantum chains under the most general non-diagonal integrable boundary conditions:

$$
\begin{aligned}
H_{X Y Z} & =\sum_{i=1}^{\mathrm{N}-1}\left(\left(1+k \operatorname{sn}^{2} \tilde{\eta}\right) \sigma_{i}^{x} \sigma_{i+1}^{x}+\left(1-k \operatorname{sn}^{2} \tilde{\eta}\right) \sigma_{i}^{y} \sigma_{i+1}^{y}+\operatorname{cn} \tilde{\eta} \operatorname{dn} \tilde{\eta} \sigma_{i}^{z} \sigma_{i+1}^{z}\right) \\
& +\frac{\operatorname{sn} \tilde{\eta}}{\operatorname{sn} \tilde{\zeta}_{-}}\left[\sigma_{1}^{z} \operatorname{cn} \tilde{\zeta}_{-} \operatorname{dn} \tilde{\zeta}_{-}+2 \kappa_{-}\left(\sigma_{1}^{x} \cosh \tau_{-}+i \sigma_{1}^{y} \sinh \tau_{-}\right)\right] \\
& +\frac{\operatorname{sn} \tilde{\eta}}{\operatorname{sn} \tilde{\zeta}_{+}}\left[\sigma_{N}^{z} \operatorname{cn} \tilde{\zeta}_{+} \operatorname{dn} \tilde{\zeta}_{+}+2 \kappa_{+}\left(\sigma_{\mathrm{N}}^{x} \cosh \tau_{+}+i \sigma_{\mathrm{N}}^{y} \sinh \tau_{+}\right)\right]
\end{aligned}
$$

Indeed, this Hamiltonian was reproduced in [79] in this homogeneous limit by the derivative of the transfer matrix (2.22).

\subsection{Properties of reflection algebra generators}

The generators of the reflection algebra $\mathcal{A}_{-}(\lambda), \mathcal{B}_{-}(\lambda), \mathcal{C}_{-}(\lambda)$ and $\mathcal{D}_{-}(\lambda)$ satisfy some important properties that we prove here. We define first the following functions:

$$
p(\lambda) \equiv \frac{2 \theta_{4}(2 \lambda+\eta \mid 2 \omega) \theta_{1}(2 \lambda-\eta \mid 2 \omega)}{\theta_{2}(0 \mid \omega)}=\theta(2 \lambda-\eta) \frac{\theta_{4}(2 \lambda+\eta \mid 2 \omega)}{\theta_{4}(2 \lambda-\eta \mid 2 \omega)},
$$

and

$$
\widehat{\mathrm{A}}_{-}(\lambda) \equiv g_{-}(\lambda) a(\lambda) d(-\lambda), \quad d(\lambda) \equiv a(\lambda-\eta), \quad a(\lambda) \equiv \prod_{n=1}^{\mathrm{N}} \theta\left(\lambda-\xi_{n}+\eta / 2\right),
$$


where:

$$
\begin{aligned}
g_{ \pm}(\lambda) \equiv & h\left(\lambda ; \zeta_{ \pm}\right)\left(\sqrt{\operatorname{sn}\left(\tilde{\lambda}+\tilde{\zeta}_{ \pm}-\tilde{\eta} / 2\right) \operatorname{sn}\left(-\tilde{\lambda}+\tilde{\zeta}_{ \pm}-\tilde{\eta} / 2\right)}\right. \\
& \left.+\kappa_{ \pm} \operatorname{sn}(2 \tilde{\lambda}-\tilde{\eta}) \frac{\sqrt{\left(1-k e^{2 \tau_{ \pm}} \operatorname{sn}^{2}(\tilde{\lambda}-\tilde{\eta} / 2)\right)\left(1-k e^{-2 \tau_{ \pm}} \operatorname{sn}^{2}(\tilde{\lambda}-\tilde{\eta} / 2)\right)}}{1-k^{2} \operatorname{sn}^{2} \tilde{\zeta}_{ \pm} \operatorname{sn}^{2}(\tilde{\lambda}-\tilde{\eta} / 2)}\right)
\end{aligned}
$$

then the following proposition holds:

Proposition 2.1. The reflection algebra generators are related by the following parity relation:

$$
\begin{aligned}
& \mathcal{A}_{-}(\lambda)=\frac{c(2 \lambda) \mathcal{D}_{-}(\lambda)+p(\lambda) \mathcal{D}_{-}(-\lambda)}{b(2 \lambda)}, \quad \mathcal{D}_{-}(\lambda)=\frac{c(2 \lambda) \mathcal{A}_{-}(\lambda)+p(\lambda) \mathcal{A}_{-}(-\lambda)}{b(2 \lambda)}, \\
& \mathcal{B}_{-}(\lambda)=\frac{a(2 \lambda) \mathcal{C}_{-}(\lambda)+p(\lambda) \mathcal{C}_{-}(-\lambda)}{d(2 \lambda)}, \quad \mathcal{C}_{-}(\lambda)=\frac{a(2 \lambda) \mathcal{B}_{-}(\lambda)+p(\lambda) \mathcal{B}_{-}(-\lambda)}{d(2 \lambda)},
\end{aligned}
$$

moreover the following identities hold:

$$
\begin{aligned}
p(\lambda) & =\frac{-c(2 \lambda) a_{-}(\lambda)+b(2 \lambda) d_{-}(\lambda)}{a_{-}(-\lambda)}=\frac{-c(2 \lambda) d_{-}(\lambda)+b(2 \lambda) a_{-}(\lambda)}{d_{-}(-\lambda)} \\
& =\frac{-a(2 \lambda) b_{-}(\lambda)+d(2 \lambda) c_{-}(\lambda)}{b_{-}(-\lambda)}=\frac{-a(2 \lambda) c_{-}(\lambda)+d(2 \lambda) b_{-}(\lambda)}{c_{-}(-\lambda)} .
\end{aligned}
$$

Moreover, it holds:

$$
\mathcal{U}_{-}^{-1}(\lambda+\eta / 2)=\frac{p(\lambda-\eta / 2)}{\operatorname{det}_{q} \mathcal{U}_{-}(\lambda)} \mathcal{U}_{-}(\eta / 2-\lambda),
$$

where in the reflection algebra generated by the elements of $\mathcal{U}_{-}(\lambda)$ the quantum determinant:

$$
\begin{aligned}
\frac{\operatorname{det}_{q} \mathcal{U}_{-}(\lambda)}{p(\lambda-\eta / 2)} & \equiv \mathcal{A}_{-}(\epsilon \lambda+\eta / 2) \mathcal{A}_{-}(\eta / 2-\epsilon \lambda)+\mathcal{B}_{-}(\epsilon \lambda+\eta / 2) \mathcal{C}_{-}(\eta / 2-\epsilon \lambda) \\
& =\mathcal{D}_{-}(\epsilon \lambda+\eta / 2) \mathcal{D}_{-}(\eta / 2-\epsilon \lambda)+\mathcal{C}_{-}(\epsilon \lambda+\eta / 2) \mathcal{B}_{-}(\eta / 2-\epsilon \lambda)
\end{aligned}
$$

where $\epsilon= \pm 1$, is central:

$$
\left[\underset{q}{\operatorname{det} \mathcal{U}_{-}}(\lambda), \mathcal{U}_{-}(\mu)\right]=0
$$

Moreover, it admits the following explicit expression:

$$
\operatorname{det}_{q} \mathcal{U}_{-}(\lambda)=p(\lambda-\eta / 2) \widehat{\mathrm{A}}_{-}(\lambda+\eta / 2) \widehat{\mathrm{A}}_{-}(-\lambda+\eta / 2) .
$$

Proof. This proposition is the 8-vertex analog of Proposition 2.1 of [1]; as in this last proposition we can derive also this 8-vertex case following Sklyanin's article [18]. The following identity holds:

$$
K_{-}^{-1}(\lambda+\eta / 2)=\frac{p(\lambda-\eta / 2)}{\operatorname{det}_{q} K_{-}(\lambda)} K_{-}(\eta / 2-\lambda),
$$

being:

$$
K_{-}(\eta / 2-\lambda) \equiv\left(\begin{array}{cc}
a_{-}(\eta / 2-\lambda) & b_{-}(\eta / 2-\lambda) \\
c_{-}(\eta / 2-\lambda) & d_{-}(\eta / 2-\lambda)
\end{array}\right)=\left(\begin{array}{cc}
d_{-}(\eta / 2+\lambda) & -b_{-}(\eta / 2+\lambda) \\
-c_{-}(\eta / 2+\lambda) & a_{-}(\eta / 2+\lambda)
\end{array}\right),
$$

where we have defined:

$$
\operatorname{det}_{q} K_{-}(\lambda)=p(\lambda-\eta / 2)\left(a_{-}(\lambda+\eta / 2) a_{-}(\eta / 2-\lambda)+b_{-}(\lambda+\eta / 2) c_{-}(\eta / 2-\lambda)\right) .
$$


Then the identity (2.31) is obtained by the following chain of identities:

$$
\begin{gathered}
\mathcal{U}_{-}(\eta / 2+\lambda) \mathcal{U}_{-}(\eta / 2-\lambda) \underset{(2.42)}{=} \underset{q}{\operatorname{det}} M_{0}(-\lambda) M_{0}(\lambda+\eta / 2) K_{-}(\lambda+\eta / 2) K_{-}(\eta / 2-\lambda) \hat{M}_{0}(\eta / 2-\lambda) \\
=\underset{q}{=} \operatorname{det} M_{0}(-\lambda) \frac{\operatorname{det}_{q} K_{-}(\lambda)}{p(\lambda-\eta / 2)} M_{0}(\lambda+\eta / 2) \hat{M}_{0}(\eta / 2-\lambda) \underset{=}{=} \frac{\operatorname{det}_{q} \mathcal{U}_{-}(\lambda)}{p(\lambda-\eta / 2)}
\end{gathered}
$$

where:

$$
\operatorname{det}_{q} \mathcal{U}_{-}(\lambda) \equiv \operatorname{det}_{q} K_{-}(\lambda) \operatorname{det}_{q} M_{0}(\lambda) \operatorname{det}_{q} M_{0}(-\lambda)
$$

and we have used that:

$$
\begin{aligned}
\hat{M}( \pm \lambda+\eta / 2) & =(-1)^{\mathrm{N}}\left(\begin{array}{cc}
D(-\eta / 2 \mp \lambda) & -B(-\eta / 2 \mp \lambda) \\
-C(-\eta / 2 \mp \lambda) & A(-\eta / 2 \mp \lambda)
\end{array}\right) \\
& =(-1)^{\mathrm{N}} \operatorname{det}_{q} M_{0}(\mp \lambda) M^{-1}(\mp \lambda+\eta / 2),
\end{aligned}
$$

where

$$
\begin{aligned}
\underset{q}{\operatorname{det}} M_{0}(\lambda) & =A(\lambda+\eta / 2) D(\lambda-\eta / 2)-B(\lambda+\eta / 2) C(\lambda-\eta / 2) \\
& =a(\lambda+\eta / 2) d(\lambda-\eta / 2)
\end{aligned}
$$

is the bulk quantum determinant, first proven to be central for the 6-vertex case in [82]. Then $\operatorname{det}_{q} \mathcal{U}_{-}(\lambda)$ is central its explicit expression (2.35) follows observing that it holds:

$$
\operatorname{det}_{q} K_{-}(\lambda)=p(\lambda-\eta / 2) g_{-}(\lambda+\eta / 2) g_{-}(-\lambda+\eta / 2) .
$$

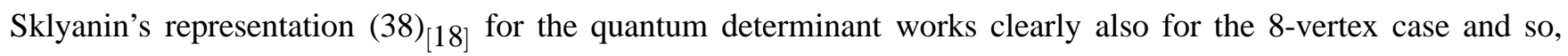
defined by

$$
\widetilde{\mathcal{U}}_{-}(\lambda) \equiv-\frac{t r_{2} R_{12}(-\eta)\left(\mathcal{U}_{-}\right)_{2}(\lambda) R_{21}(2 \lambda)}{\theta_{1}(\eta \mid \omega)}=\left(\begin{array}{cc}
\widetilde{\mathcal{D}}_{-}(\lambda) & -\widetilde{\mathcal{B}}_{-}(\lambda) \\
-\widetilde{\mathcal{C}}_{-}(\lambda) & \widetilde{\mathcal{A}}_{-}(\lambda)
\end{array}\right)
$$

the "algebraic adjoint" of the boundary monodromy matrix $\mathcal{U}_{-}(\lambda), \widetilde{\mathcal{U}}_{-}(\lambda)$ admits the following explict form in the 8-vertex case:

$$
\widetilde{\mathcal{U}}_{-}(\lambda)=\left(\begin{array}{cc}
\mathcal{D}_{-}(\lambda) \mathrm{b}(2 \lambda)-\mathcal{A}_{-}(\lambda) \mathrm{c}(2 \lambda) & \mathcal{C}_{-}(\lambda) \mathrm{d}(2 \lambda)-\mathcal{B}_{-}(\lambda) \mathrm{a}(2 \lambda) \\
\mathcal{B}_{-}(\lambda) \mathrm{d}(2 \lambda)-\mathcal{C}_{-}(\lambda) \mathrm{a}(2 \lambda) & \mathcal{A}_{-}(\lambda) \mathrm{b}(2 \lambda)-\mathcal{D}_{-}(\lambda) \mathrm{c}(2 \lambda)
\end{array}\right)
$$

and it satisfies the identity (41) $[18$ :

$$
\tilde{\mathcal{U}}_{-}(\lambda-\eta / 2) \mathcal{U}_{-}(\lambda+\eta / 2)=\operatorname{det}_{q} \mathcal{U}_{-}(\lambda),
$$

and so from the identity (2.31) it follows:

$$
\widetilde{\mathcal{U}}_{-}(\lambda)=p(\lambda) \mathcal{U}_{-}(-\lambda)
$$

which by using (2.45) implies the symmetry properties (2.27) and (2.28). Finally, let us remark that the identities in (2.29) and (2.30) can be proven by direct computations and in fact they just coincides with (2.27) and (2.28) for the scalar case $\mathrm{N}=0$.

Similar statements hold for the reflection algebra generated by $\mathcal{U}_{+}(\lambda)$, as they are simply consequences of the previous proposition being $\mathcal{U}_{+}^{t_{0}}(-\lambda)$ solution of the same reflection equation of $\mathcal{U}_{-}(\lambda)$. 
Lemma 2.1. The most general boundary transfer matrix $\mathcal{T}(\lambda)$ is even in the spectral parameter $\lambda$ :

$$
\mathcal{T}(-\lambda)=\mathcal{T}(\lambda)
$$

Proof. The identity (2.48) can be proven by using the following list of the identities:

$$
\begin{aligned}
& \mathcal{T}(-\lambda)=\operatorname{tr}_{0}\left\{K_{+}(-\lambda) \mathcal{U}_{-}(-\lambda)\right\}=\frac{\operatorname{tr}_{0}\left\{K_{+}(-\lambda) \widetilde{\mathcal{U}}_{-}(\lambda)\right\}}{p(\lambda)} \\
& =p^{-1}(\lambda)\left(\mathcal{A}_{-}(\lambda) a_{+}(\lambda) \frac{d_{+}(-\lambda) \mathrm{b}(2 \lambda)-a_{+}(-\lambda) \mathrm{c}(2 \lambda)}{a_{+}(\lambda)}+\mathcal{D}_{-}(\lambda) d_{+}(\lambda) \frac{a_{+}(-\lambda) \mathrm{b}(2 \lambda)-d_{+}(-\lambda) \mathrm{c}(2 \lambda)}{d_{+}(\lambda)}\right. \\
& \left.+\mathcal{B}_{-}(\lambda) c_{+}(\lambda) \frac{b_{+}(-\lambda) \mathrm{d}(2 \lambda)-c_{+}(-\lambda) \mathrm{d}(2 \lambda)}{c_{+}(\lambda)}+\mathcal{C}_{-}(\lambda) b_{+}(\lambda) \frac{c_{+}(-\lambda) \mathrm{d}(2 \lambda)-b_{+}(-\lambda) \mathrm{d}(2 \lambda)}{b_{+}(\lambda)}\right) \\
& =\mathcal{A}_{-}(\lambda) a_{+}(\lambda)+\mathcal{D}_{-}(\lambda) d_{+}(\lambda)+\mathcal{B}_{-}(\lambda) c_{+}(\lambda)+\mathcal{C}_{-}(\lambda) b_{+}(\lambda)=\mathcal{T}(\lambda)
\end{aligned}
$$

once we observe that the following identities holds:

$$
\begin{aligned}
p(\lambda) & =\frac{-\mathrm{c}(2 \lambda) a_{+}(-\lambda)+\mathrm{b}(2 \lambda) d_{+}(-\lambda)}{a_{+}(\lambda)}=\frac{-\mathrm{c}(2 \lambda) d_{+}(-\lambda)+\mathrm{b}(2 \lambda) a_{+}(-\lambda)}{d_{+}(\lambda)} \\
& =\frac{-\mathrm{a}(2 \lambda) b_{+}(-\lambda)+\mathrm{d}(2 \lambda) c_{+}(-\lambda)}{b_{+}(\lambda)}=\frac{-\mathrm{a}(2 \lambda) c_{+}(-\lambda)+\mathrm{d}(2 \lambda) b_{+}(-\lambda)}{c_{+}(\lambda)},
\end{aligned}
$$

as a direct consequence of the identities (2.29) $-(2.30)$ being:

$$
\begin{array}{ll}
a_{+}\left(-\lambda \mid \zeta_{+}\right)=d_{-}\left(\lambda \mid \zeta_{-}^{\prime}\right), & c_{+}\left(-\lambda \mid \zeta_{+}, \kappa_{+}, \tau_{+}\right)=-c_{-}\left(\lambda \mid \zeta_{-}^{\prime}, \kappa_{-}^{\prime}, \tau_{-}^{\prime}\right) \\
d_{+}\left(-\lambda \mid \zeta_{+}\right)=a_{-}\left(\lambda \mid \zeta_{-}^{\prime}\right), & b_{+}\left(-\lambda \mid \zeta_{+}, \kappa_{ \pm}, \tau_{ \pm}\right)=-b_{-}\left(\lambda \mid \zeta_{-}^{\prime}, \kappa_{-}^{\prime}, \tau_{-}^{\prime}\right)
\end{array}
$$

once we identify $\zeta_{-}^{\prime} \equiv \zeta_{+}, \kappa_{-}^{\prime} \equiv \kappa_{+}, \tau_{-}^{\prime} \equiv \tau_{+}$.

\section{Baxter's gauge transformations and central properties}

\subsection{Notations}

Let us introduce the following $2 \times 2$ matrices:

$$
\begin{array}{rlr}
\bar{G}(\lambda \mid \beta) & \equiv\left(X_{\beta}(\lambda), Y_{\beta}(\lambda)\right), & \tilde{G}(\lambda \mid \beta) \equiv\left(X_{\beta+1}(\lambda), Y_{\beta-1}(\lambda)\right) \\
\bar{G}^{-1}(\lambda \mid \beta) & \equiv\left(\begin{array}{c}
\bar{Y}_{\beta}(\lambda) \\
\bar{X}_{\beta}(\lambda)
\end{array}\right), & \tilde{G}^{-1}(\lambda \mid \beta) \equiv\left(\begin{array}{c}
\tilde{Y}_{\beta-1}(\lambda) \\
\tilde{X}_{\beta+1}(\lambda)
\end{array}\right)
\end{array}
$$

where:

$$
X_{\beta}(\lambda) \equiv\left(\begin{array}{c}
\theta_{2}(\lambda+(\alpha+\beta) \eta \mid 2 \omega) \\
\theta_{3}(\lambda+(\alpha+\beta) \eta \mid 2 \omega)
\end{array}\right), \quad Y_{\beta}(\lambda) \equiv\left(\begin{array}{c}
\theta_{2}(\lambda+(\alpha-\beta) \eta \mid 2 \omega) \\
\theta_{3}(\lambda+(\alpha-\beta) \eta \mid 2 \omega)
\end{array}\right)
$$

and

$$
\begin{aligned}
\bar{X}_{\beta}(\lambda) & \equiv \frac{\left(\theta_{3}(\lambda+(\alpha+\beta) \eta \mid 2 \omega)-\theta_{2}(\lambda+(\alpha+\beta) \eta \mid 2 \omega)\right)}{\theta(\lambda+\alpha \eta) \theta(\beta \eta)} \\
\tilde{X}_{\beta}(\lambda) & =\frac{\theta(\lambda+\alpha \eta) \theta(\beta \eta)}{\theta(\lambda+(\alpha+1) \eta) \theta((\beta-1) \eta)} \bar{X}_{\beta}(\lambda) \\
\bar{Y}_{\beta}(\lambda) & \equiv \frac{\left(-\theta_{3}(\lambda+(\alpha-\beta) \eta \mid 2 \omega) \theta_{2}(\lambda+(\alpha-\beta) \eta \mid 2 \omega)\right)}{\theta(\lambda+\alpha \eta) \theta(\beta \eta)} \\
\tilde{Y}_{\beta}(\lambda) & =\frac{\theta(\lambda+\alpha \eta) \theta(\beta \eta)}{\theta(\lambda+(\alpha+1) \eta) \theta((1+\beta) \eta)} \bar{Y}_{\beta}(\lambda) .
\end{aligned}
$$


Here, $\alpha$ and $\beta$ are arbitrary complex number and for simplicity we have introduced the notation $\theta(\lambda) \equiv \theta_{1}(\lambda \mid \omega)$ and we omit the index $\alpha$ as it does not play an explicit role in the following. These covectors/vectors satisfy the following relations:

$$
\begin{aligned}
& \begin{array}{ll}
\bar{Y}_{\beta}(\lambda) X_{\beta}(\lambda)=1, & \bar{Y}_{\beta}(\lambda) Y_{\beta}(\lambda)=0, \\
\bar{X}_{\beta}(\lambda) X_{\beta}(\lambda)=0, & \bar{X}_{\beta}(\lambda) Y_{\beta}(\lambda)=1,
\end{array} \quad \text { and } X_{\beta}(\lambda) \bar{Y}_{\beta}(\lambda)+Y_{\beta}(\lambda) \bar{X}_{\beta}(\lambda)=I \equiv\left(\begin{array}{ll}
1 & 0 \\
0 & 1
\end{array}\right), \\
& \begin{array}{ll}
\tilde{Y}_{\beta-1}(\lambda) X_{\beta+1}(\lambda)=1, & \tilde{Y}_{\beta-1}(\lambda) Y_{\beta-1}(\lambda)=0, \\
\tilde{X}_{\beta+1}(\lambda) X_{\beta+1}(\lambda)=0, & \tilde{X}_{\beta+1}(\lambda) Y_{\beta-1}(\lambda)=1,
\end{array} \quad \text { and } X_{\beta+1}(\lambda) \tilde{Y}_{\beta-1}(\lambda)+Y_{\beta-1}(\lambda) \tilde{X}_{\beta+1}(\lambda)=I .
\end{aligned}
$$

\subsection{Baxter's gauge transformation}

The Baxter's gauge transformations, first introduce in [57], have the following matrix form:

$$
R_{0 a}^{(8 \mathrm{~V})}\left(\lambda_{12}\right) S_{0}\left(\lambda_{1} \mid \alpha, \beta\right) S_{a}\left(\lambda_{2} \mid \alpha, \beta+\sigma_{0}^{z}\right)=S_{a}\left(\lambda_{2} \mid \alpha, \beta\right) S_{0}\left(\lambda_{1} \mid \alpha, \beta+\sigma_{a}^{z}\right) R_{0 a}^{(6 \mathrm{VD})}\left(\lambda_{12} \mid \beta\right)
$$

where:

$$
S_{0}(\lambda \mid \alpha, \beta) \equiv\left(Y_{\beta}(\lambda) \quad X_{\beta}(\lambda)\right)
$$

In (3.10) $R_{12}^{(6 \mathrm{VD})}\left(\lambda_{12} \mid \beta\right)$ is the elliptic solution of the following dynamical 6-vertex Yang-Baxter equation [58]:

$$
R_{12}^{(6 \mathrm{VD})}\left(\lambda_{12} \mid \beta+\sigma_{a}^{z}\right) R_{1 a}^{(6 \mathrm{VD})}\left(\lambda_{1} \mid \beta\right) R_{2 a}^{(6 \mathrm{VD})}\left(\lambda_{2} \mid \beta+\sigma_{1}^{z}\right)=R_{2 a}^{(6 \mathrm{VD})}\left(\lambda_{2} \mid \beta\right) R_{1 a}^{(6 \mathrm{VD})}\left(\lambda_{1} \mid \beta+\sigma_{2}^{z}\right) R_{12}^{(6 \mathrm{VD})}\left(\lambda_{12} \mid \beta\right)
$$

and it has the form:

$$
R_{12}^{(6 \mathrm{VD})}(\lambda \mid \beta)=\left(\begin{array}{cccc}
\mathrm{a}(\lambda) & 0 & 0 & 0 \\
0 & \mathrm{~b}(\lambda \mid \beta) & \mathrm{c}(\lambda \mid \beta) & 0 \\
0 & \mathrm{c}(\lambda \mid-\beta) & \mathrm{b}(\lambda \mid-\beta) & 0 \\
0 & 0 & 0 & \mathrm{a}(\lambda)
\end{array}\right)
$$

where $\mathrm{a}(\lambda), \mathrm{b}(\lambda \mid \beta)$ and $\mathrm{c}(\lambda \mid \beta)$ are defined by:

$$
\mathrm{a}(\lambda)=\theta(\lambda+\eta), \quad \mathrm{b}(\lambda \mid \beta)=\frac{\theta(\lambda) \theta((\beta+1) \eta)}{\theta(\beta \eta)}, \quad \mathrm{c}(\lambda \mid \beta)=\frac{\theta(\eta) \theta(\beta \eta+\lambda)}{\theta(\beta \eta)}
$$

Historically, Baxter has used first a vectorial representation for these transformations, which explicitly reads:

$$
\begin{aligned}
R_{12}\left(\lambda_{12}\right) X_{1, \beta}\left(\lambda_{1}\right) X_{2, \beta-1}\left(\lambda_{2}\right) & =\mathrm{a}\left(\lambda_{12}\right) X_{2, \beta}\left(\lambda_{2}\right) X_{1, \beta-1}\left(\lambda_{1}\right), \\
R_{12}\left(\lambda_{12}\right) X_{1, \beta}\left(\lambda_{1}\right) Y_{2, \beta-1}\left(\lambda_{2}\right) & =\mathrm{b}\left(\lambda_{12} \mid-\beta\right) Y_{2, \beta}\left(\lambda_{2}\right) X_{1, \beta+1}\left(\lambda_{1}\right) \\
& +\mathrm{c}\left(\lambda_{12} \mid\right) X_{2, \beta}\left(\lambda_{2}\right) Y_{1, \beta-1}\left(\lambda_{1}\right), \\
R_{12}\left(\lambda_{12}\right) Y_{1, \beta}\left(\lambda_{1}\right) X_{2, \beta+1}\left(\lambda_{2}\right) & =\mathrm{b}\left(\lambda_{12} \mid \beta\right) X_{2, \beta}\left(\lambda_{2}\right) Y_{1, \beta-1}\left(\lambda_{1}\right) \\
& +\mathrm{c}\left(\lambda_{12} \mid-\beta\right) Y_{2, \beta}\left(\lambda_{2}\right) X_{1, \beta+1}\left(\lambda_{1}\right), \\
R_{12}\left(\lambda_{12}\right) Y_{1, \beta}\left(\lambda_{1}\right) Y_{2, \beta+1}\left(\lambda_{2}\right) & =\mathrm{a}\left(\lambda_{12}\right) Y_{2, \beta}\left(\lambda_{2}\right) Y_{1, \beta+1}\left(\lambda_{1}\right),
\end{aligned}
$$

this clarifies the original use of the terminology intertwining vectors for these gauge transformations. 


\subsection{Gauge transformed boundary operators and their properties}

\subsubsection{Definitions}

Let us define the following bulk gauge transformed monodromy matrices:

$$
\begin{aligned}
& M(\lambda \mid \beta) \equiv \tilde{G}_{\beta}^{-1}(\lambda-\eta / 2) M(\lambda) \tilde{G}_{\beta+\mathrm{N}}(\lambda-\eta / 2) \equiv\left(\begin{array}{cc}
A(\lambda \mid \beta) & B(\lambda \mid \beta) \\
C(\lambda \mid \beta) & D(\lambda \mid \beta)
\end{array}\right), \\
& \hat{M}(\lambda \mid \beta) \equiv \bar{G}_{\beta+\mathrm{N}}^{-1}(\eta / 2-\lambda) \hat{M}(\lambda) \bar{G}_{\beta}(\eta / 2-\lambda) \equiv\left(\begin{array}{cc}
\bar{A}(\lambda \mid \beta) & \bar{B}(\lambda \mid \beta) \\
\bar{C}(\lambda \mid \beta) & \bar{D}(\lambda \mid \beta)
\end{array}\right),
\end{aligned}
$$

and the following boundary one:

$$
\mathrm{U}_{-}(\lambda \mid \beta) \equiv\left(\begin{array}{cc}
\widehat{\mathcal{A}}_{-}(\lambda \mid \beta+2) & \widehat{\mathcal{B}}_{-}(\lambda \mid \beta) \\
\widehat{\mathcal{C}}_{-}(\lambda \mid \beta+2) & \widehat{\mathcal{D}}_{-}(\lambda \mid \beta)
\end{array}\right) \equiv \tilde{G}^{-1}(\lambda-\eta / 2 \mid \beta) \mathcal{U}_{-}(\lambda) \tilde{G}(\eta / 2-\lambda \mid \beta) .
$$

\subsubsection{Main symmetries}

The following rescaled gauge transformed boundary operators:

$$
\begin{aligned}
\mathcal{A}_{-}(\lambda \mid \beta) & \equiv r(\lambda) \widehat{\mathcal{A}}_{-}(\lambda \mid \beta), \quad \mathcal{B}_{-}(\lambda \mid \beta) \equiv r(\lambda) \widehat{\mathcal{B}}_{-}(\lambda \mid \beta), \\
\mathcal{C}_{-}(\lambda \mid \beta) & \equiv r(\lambda) \widehat{\mathcal{C}}_{-}(\lambda \mid \beta), \quad \mathcal{D}_{-}(\lambda \mid \beta) \equiv r(\lambda) \widehat{\mathcal{D}}_{-}(\lambda \mid \beta), \\
r(\lambda) & \equiv \theta_{4}(2 \lambda-\eta \mid 2 \omega) \theta(\lambda+(\alpha+1 / 2) \eta)
\end{aligned}
$$

satisfy the following central properties:

Proposition 3.1. $\mathcal{A}_{-}(\lambda \mid \beta)$ and $\mathcal{D}_{-}(\lambda \mid \beta)$ satisfies the following interrelated parity relations:

$$
\begin{aligned}
& \mathcal{A}_{-}(\lambda \mid \beta)=-\frac{\theta(\eta) \theta(2 \lambda-(\beta-1) \eta)}{\theta(2 \lambda) \theta((\beta-2) \eta)} \mathcal{D}_{-}(\lambda \mid \beta)+\frac{\theta(2 \lambda-\eta) \theta((\beta-1) \eta)}{\theta(2 \lambda) \theta((\beta-2) \eta)} \mathcal{D}_{-}(-\lambda \mid \beta), \\
& \mathcal{D}_{-}(\lambda \mid \beta)=\frac{\theta(\eta) \theta(2 \lambda+(\beta-1) \eta)}{\theta(2 \lambda) \theta(\beta \eta)} \mathcal{A}_{-}(\lambda \mid \beta)+\frac{\theta(2 \lambda-\eta) \theta((\beta-1) \eta)}{\theta(2 \lambda) \theta(\beta \eta)} \mathcal{A}_{-}(-\lambda \mid \beta),
\end{aligned}
$$

while $\mathcal{B}_{-}(\lambda \mid \beta)$ and $\mathcal{C}_{-}(\lambda \mid \beta)$ satisfy the following independent parity relations:

$$
\mathcal{B}_{-}(-\lambda \mid \beta)=-\frac{\theta(2 \lambda+\eta)}{\theta(2 \lambda-\eta)} \mathcal{B}_{-}(\lambda \mid \beta), \mathcal{C}_{-}(-\lambda \mid \beta)=-\frac{\theta(2 \lambda+\eta)}{\theta(2 \lambda-\eta)} \mathcal{C}_{-}(\lambda \mid \beta)
$$

Moreover, it holds:

$$
\mathrm{U}_{-}^{-1}(\lambda+\eta / 2 \mid \beta)=\frac{\tilde{\mathbf{U}}_{-}(\lambda-\eta / 2 \mid \beta)}{\operatorname{det}_{q} \mathcal{U}_{-}(\lambda)}=\frac{p(\lambda-\eta / 2)}{\operatorname{det}_{q} \mathcal{U}_{-}(\lambda)} \mathbf{U}_{-}(\eta / 2-\lambda \mid \beta),
$$

where:

$$
\tilde{U}_{-}(\lambda \mid \beta) \equiv \tilde{G}^{-1}(-\lambda-\eta / 2 \mid \beta) \tilde{\mathcal{U}}_{-}(\lambda) \tilde{G}(\eta / 2+\lambda \mid \beta)
$$

and the quantum determinant admits the representation, for both $\epsilon= \pm 1$ :

$$
\begin{aligned}
& \frac{\operatorname{det}_{q} \mathcal{U}_{-}(\lambda) r(\lambda+\eta / 2) r(-\lambda+\eta / 2)}{p(\lambda-\eta / 2)}= \\
& =\mathcal{A}_{-}(\epsilon \lambda+\eta / 2 \mid \beta+2) \mathcal{A}_{-}(\eta / 2-\epsilon \lambda \mid \beta+2)+\mathcal{B}_{-}(\epsilon \lambda+\eta / 2 \mid \beta) \mathcal{C}_{-}(\eta / 2-\epsilon \lambda \mid \beta+2) \\
& =\mathcal{D}_{-}(\epsilon \lambda+\eta / 2 \mid \beta) \mathcal{D}_{-}(\eta / 2-\epsilon \lambda \mid \beta)+\mathcal{C}_{-}(\epsilon \lambda+\eta / 2 \mid \beta+2) \mathcal{B}_{-}(\eta / 2-\epsilon \lambda \mid \beta) \text {. }
\end{aligned}
$$


Proof. Let us first prove the equation (3.28), by definition it holds:

$$
\tilde{U}_{-}(\lambda-\eta / 2 \mid \beta) \equiv \tilde{G}_{\beta}^{-1}(-\lambda) \tilde{\mathcal{U}}_{-}(\lambda-\eta / 2) \tilde{G}_{\beta}(\lambda), \mathbf{U}_{-}(\lambda+\eta / 2 \mid \beta) \equiv \tilde{G}_{\beta}^{-1}(\lambda) \mathcal{U}_{-}(\lambda+\eta / 2) \tilde{G}_{\beta}(-\lambda),
$$

and then:

$$
\begin{aligned}
\mathrm{U}_{-}(\lambda+\eta / 2 \mid \beta) \tilde{\mathrm{U}}_{-}(\lambda-\eta / 2 \mid \beta) & =\tilde{G}_{\beta}^{-1}(\lambda) \mathcal{U}_{-}(\lambda+\eta / 2) \tilde{\mathcal{U}}_{-}(\lambda-\eta / 2) \tilde{G}_{\beta}(\lambda) \\
& =\tilde{G}_{\beta}^{-1}(\lambda) \operatorname{det}_{q} \mathcal{U}_{-}(\lambda) \tilde{G}_{\beta}(\lambda) \\
& =\operatorname{det}_{q} \mathcal{U}_{-}(\lambda),
\end{aligned}
$$

and similarly:

$$
\begin{aligned}
\tilde{\mathrm{U}}_{-}(\lambda-\eta / 2 \mid \beta) \mathrm{U}_{-}(\lambda+\eta / 2 \mid \beta) & =\tilde{G}_{\beta}^{-1}(-\lambda) \tilde{\mathcal{U}}_{-}(\lambda-\eta / 2) \mathcal{U}_{-}(\lambda+\eta / 2) \tilde{G}_{\beta}(-\lambda) \\
& =\tilde{G}_{\beta}^{-1}(-\lambda) \operatorname{det}_{q} \mathcal{U}_{-}(\lambda) \tilde{G}_{\beta}(-\lambda) \\
& =\operatorname{det}_{q} \mathcal{U}_{-}(\lambda) .
\end{aligned}
$$

From these identities the expressions for the quantum determinant in terms of gauge transformed operators directly follow. Moreover, defined:

$$
f_{\alpha}(\lambda) \equiv \frac{\theta((\alpha+1 / 2) \eta+\lambda)}{\theta((\alpha+1 / 2) \eta-\lambda)}
$$

the identities:

$$
\begin{aligned}
& \left(\tilde{\mathrm{U}}_{-}(\lambda \mid \beta)\right)_{12}=-f_{\alpha}(\lambda) \theta(2 \lambda+\eta) \widehat{\mathcal{B}}_{-}(\lambda \mid \beta), \quad\left(\tilde{\mathrm{U}}_{-}(\lambda \mid \beta)\right)_{21}=-f_{\alpha}(\lambda) \theta(2 \lambda+\eta) \widehat{\mathcal{C}}_{-}(\lambda \mid \beta), \\
& \left(\tilde{\mathrm{U}}_{-}(\lambda \mid \beta)\right)_{22}=f_{\alpha}(\lambda)\left(\frac{\theta(2 \lambda) \theta((\beta-2) \eta)}{\theta((\beta-1) \eta)} \widehat{\mathcal{A}}_{-}(\lambda \mid \beta)+\frac{\theta(\eta) \theta(2 \lambda-(\beta-1) \eta)}{\theta((\beta-1) \eta)} \widehat{\mathcal{D}}_{-}(\lambda \mid \beta)\right),
\end{aligned}
$$

can be shown by direct computation expanding both the elements of $\tilde{U}_{-}(\lambda \mid \beta)$ and $\mathcal{U}_{-}(\lambda \mid \beta)$ in terms of the ungauged elements of $\mathcal{U}_{-}(\lambda)$. Then the formulae (3.25) and (3.27) are simply derived by using the above identities and the identity:

$$
\begin{aligned}
\tilde{U}_{-}(\lambda \mid \beta) & =p(\lambda)\left(\begin{array}{c}
\tilde{Y}_{\beta-1}(-\lambda-\eta / 2) \\
\tilde{X}_{\beta+1}(-\lambda-\eta / 2)
\end{array}\right) \mathcal{U}_{-}(-\lambda)\left(X_{\beta+1}(\eta / 2+\lambda) Y_{\beta-1}(\eta / 2+\lambda)\right) \\
& =p(\lambda) \mathrm{U}_{-}(-\lambda \mid \beta) .
\end{aligned}
$$

\subsubsection{Commutations relations}

All the commutation relations that we need to define the left and right SOV representations of the gauge transformed generators of the reflection algebra are contained in the following lemma.

Lemma 3.1. The following commutation relations are satisfied:

$$
\mathcal{B}_{-}\left(\lambda_{2} \mid \beta\right) \mathcal{B}_{-}\left(\lambda_{1} \mid \beta-2\right)=\mathcal{B}_{-}\left(\lambda_{1} \mid \beta\right) \mathcal{B}_{-}\left(\lambda_{2} \mid \beta-2\right),
$$


and

$$
\begin{aligned}
\mathcal{A}_{-}\left(\lambda_{2} \mid \beta+2\right) \mathcal{B}_{-}\left(\lambda_{1} \mid \beta\right)= & \frac{\theta\left(\lambda_{1}-\lambda_{2}+\eta\right) \theta\left(\lambda_{2}+\lambda_{1}-\eta\right)}{\theta\left(\lambda_{1}-\lambda_{2}\right) \theta\left(\lambda_{1}+\lambda_{2}\right)} \mathcal{B}_{-}\left(\lambda_{1} \mid \beta\right) \mathcal{A}_{-}\left(\lambda_{2} \mid \beta\right) \\
& +\frac{\theta\left(\lambda_{1}+\lambda_{2}-\eta\right) \theta\left(\lambda_{1}-\lambda_{2}+(\beta-1) \eta\right) \theta(\eta)}{\theta\left(\lambda_{2}-\lambda_{1}\right) \theta\left(\lambda_{1}+\lambda_{2}\right) \theta((\beta-1) \eta)} \mathcal{B}_{-}\left(\lambda_{2} \mid \beta\right) \mathcal{A}_{-}\left(\lambda_{1} \mid \beta\right) \\
& +\frac{\theta(\eta) \theta\left(\lambda_{1}+\lambda_{2}-\beta \eta\right)}{\theta\left(\lambda_{1}+\lambda_{2}\right) \theta((\beta-1) \eta)} \mathcal{B}_{-}\left(\lambda_{2} \mid \beta\right) \mathcal{D}_{-}\left(\lambda_{1} \mid \beta\right),
\end{aligned}
$$

and

$$
\begin{aligned}
\mathcal{B}_{-}\left(\lambda_{1} \mid \beta\right) \mathcal{D}_{-}\left(\lambda_{2} \mid \beta\right)= & \frac{\theta\left(\lambda_{1}-\lambda_{2}+\eta\right) \theta\left(\lambda_{2}+\lambda_{1}-\eta\right)}{\theta\left(\lambda_{1}-\lambda_{2}\right) \theta\left(\lambda_{1}+\lambda_{2}\right)} \mathcal{D}_{-}\left(\lambda_{2} \mid \beta+2\right) \mathcal{B}_{-}\left(\lambda_{1} \mid \beta\right) \\
& -\frac{\theta\left(\lambda_{2}-\lambda_{1}+(1+\beta) \eta\right) \theta\left(\lambda_{2}+\lambda_{1}-\eta\right)}{\theta\left(\lambda_{1}-\lambda_{2}\right) \theta\left(\lambda_{2}+\lambda_{1}\right) \theta((1+\beta) \eta)} \mathcal{D}_{-}\left(\lambda_{1} \mid \beta+2\right) \mathcal{B}_{-}\left(\lambda_{2} \mid \beta\right) \\
& -\frac{\theta(\eta) \theta\left(\lambda_{2}+\lambda_{1}+\beta \eta\right)}{\theta\left(\lambda_{2}+\lambda_{1}\right) \theta((1+\beta) \eta)} \mathcal{A}_{-}\left(\lambda_{1} \mid \beta+2\right) \mathcal{B}_{-}\left(\lambda_{2} \mid \beta\right)
\end{aligned}
$$

and

$$
\begin{aligned}
\mathcal{A}_{-}\left(\lambda_{1} \mid \beta+2\right) \mathcal{A}_{-} & \left(\lambda_{2} \mid \beta+2\right)-\frac{\theta(\eta) \theta\left(\lambda_{1}+\lambda_{2}-\beta \eta\right)}{\theta\left(\lambda_{1}+\lambda_{2}\right) \theta((\beta-1) \eta)} \mathcal{B}_{-}\left(\lambda_{1} \mid \beta\right) \mathcal{C}_{-}\left(\lambda_{2} \mid \beta+2\right)= \\
& \mathcal{A}_{-}\left(\lambda_{2} \mid \beta+2\right) \mathcal{A}_{-}\left(\lambda_{1} \mid \beta+2\right)-\frac{\theta(\eta) \theta\left(\lambda_{1}+\lambda_{2}-\beta \eta\right)}{\theta\left(\lambda_{1}+\lambda_{2}\right) \theta((\beta-1) \eta)} \mathcal{B}_{-}\left(\lambda_{2} \mid \beta\right) \mathcal{C}_{-}\left(\lambda_{1} \mid \beta+2\right) .
\end{aligned}
$$

Proof. The first two commutation relations were first presented in the paper [64] and the others can be derived similarly by using the Baxter's gauge transformation properties and the reflection equation.

Note that these commutation relations for the gauge transformed generators of the 8-vertex reflection algebra exactly coincides with those of the gauge transformed 6-vertex ones once we transform the function $\theta()$ in $\sinh ()$. This observation and the remark that the first coefficients both in (3.41) and in (3.42) do not depend from the gauge parameters and coincide (under the same elliptic to trigonometric transformation) with those appearing in commutation relations of the original 6-vertex reflection algebra are at the basis of the strong similarity in all the SOV representation of reflection algebra generators. This will appear clearly comparing the SOV representation of the gauge transformed generators in the 8-vertex reflection algebra here derived with those of the 6-vertex reflection algebra in the gauged [2] and ungauged [1] cases.

\subsection{4 $\beta$-parity relations}

Lemma 3.2. The gauge transformed generators satisfy the following symmetry:

$$
\mathcal{U}_{-}(\lambda \mid-\beta+2)=\sigma^{x} \mathcal{U}_{-}(\lambda \mid \beta) \sigma^{x}
$$

which in terms of matrix elements reads:

$$
\mathcal{B}_{-}(\lambda \mid \beta)=\mathcal{C}_{-}(\lambda \mid-\beta+2), \quad \mathcal{A}_{-}(\lambda \mid \beta)=\mathcal{D}_{-}(\lambda \mid-\beta+2) .
$$

Proof. The proof is a trivial consequence of the following simple identities:

$$
\tilde{Y}_{\beta}(\lambda)=\tilde{X}_{-\beta}(\lambda), \quad Y_{\beta}(\lambda)=X_{-\beta}(\lambda)
$$


e.g. we have that:

$$
\begin{aligned}
\widehat{\mathcal{B}}_{-}(\lambda \mid \beta) & =\tilde{Y}_{\beta-1}(\lambda-\eta / 2) \mathcal{U}_{-}(\lambda) Y_{\beta-1}(\eta / 2-\lambda) \\
& =\tilde{X}_{(-\beta+2)-1}(\lambda-\eta / 2) \mathcal{U}_{-}(\lambda) X_{(-\beta+2)-1}(\eta / 2-\lambda) \\
& =\widehat{\mathcal{C}}_{-}(\lambda \mid-\beta+2) .
\end{aligned}
$$

\subsection{Transfer matrix representations in terms of gauge transformed boundary operators}

Let us introduce the vectors:

$$
\begin{aligned}
\hat{Y}_{\beta-1}(\lambda) & =\frac{\theta((2+\beta) \eta) Y_{\beta-1}(\lambda)}{\theta((1+\beta) \eta) \theta(\lambda+(\alpha+2) \eta) \theta_{4}(2 \lambda \mid 2 \omega)}, \quad \underline{Y}_{\beta}(\lambda)=\frac{\bar{Y}_{\beta}(\lambda)}{\theta_{4}(2 \lambda \mid 2 \omega) \theta(-\lambda+(\alpha+1) \eta)}, \\
\hat{X}_{\beta+3}(\lambda) & =\frac{\theta(\beta \eta) X_{\beta+3}(\lambda)}{\theta((1+\beta) \eta) \theta(\lambda+(\alpha+2) \eta) \theta_{4}(2 \lambda \mid 2 \omega)}, \quad \underline{X}_{\beta}(\lambda)=\frac{\bar{X}_{\beta}(\lambda)}{\theta_{4}(2 \lambda \mid 2 \omega) \theta(-\lambda+(\alpha+1) \eta)}
\end{aligned}
$$

and the following two gauge transformations on the boundary matrix $K_{+}$:

$$
\begin{array}{ll}
K_{+}^{(L)}(\lambda \mid \beta)_{11} \equiv \tilde{Y}_{\beta-1}(\eta / 2-\lambda) K_{+}(\lambda) \hat{X}_{\beta+3}(\lambda-\eta / 2), & K_{+}^{(L)}(\lambda \mid \beta)_{12} \equiv \tilde{Y}_{\beta+1}(\eta / 2-\lambda) K_{+}(\lambda) \hat{Y}_{\beta-1}(\lambda-\eta / 2), \\
K_{+}^{(L)}(\lambda \mid \beta)_{21} \equiv \tilde{X}_{\beta+1}(\eta / 2-\lambda) K_{+}(\lambda) \hat{X}_{\beta+3}(\lambda-\eta / 2), & K_{+}^{(L)}(\lambda \mid \beta)_{22} \equiv \tilde{X}_{\beta+3}(\eta / 2-\lambda) K_{+}(\lambda) \hat{Y}_{\beta-1}(\lambda-\eta / 2),
\end{array}
$$

and

$$
\begin{array}{ll}
K_{+}^{(R)}(\lambda \mid \beta)_{11} \equiv \underline{Y}_{\beta+1}(\eta / 2-\lambda) K_{+}(\lambda) X_{\beta+1}(\lambda-\eta / 2), & K_{+}^{(R)}(\lambda \mid \beta)_{12} \equiv \underline{Y}_{\beta+1}(\eta / 2-\lambda) K_{+}(\lambda) Y_{\beta-1}(\lambda-\eta / 2), \\
K_{+}^{(R)}(\lambda \mid \beta)_{21} \equiv \underline{X}_{\beta+1}(\eta / 2-\lambda) K_{+}(\lambda) X_{\beta+3}(\lambda-\eta / 2), & K_{+}^{(R)}(\lambda \mid \beta)_{22} \equiv \underline{X}_{\beta+1}(\eta / 2-\lambda) K_{+}(\lambda) Y_{\beta+1}(\lambda-\eta / 2),
\end{array}
$$

then the following proposition holds:

Proposition 3.2. In terms of the gauge transformed reflection algebra generators the boundary transfer matrix $\mathcal{T}(\lambda)$ admit the decompositions:

$$
\begin{aligned}
\mathcal{T}(\lambda) & =K_{+}^{(L)}(\lambda \mid \beta)_{11} \mathcal{A}_{-}(\lambda \mid \beta+2)+K_{+}^{(L)}(\lambda \mid \beta)_{21} \mathcal{B}_{-}(\lambda \mid \beta) \\
& +K_{+}^{(L)}(\lambda \mid \beta)_{12} \mathcal{C}_{-}(\lambda \mid \beta+4)+K_{+}^{(L)}(\lambda \mid \beta)_{22} \mathcal{D}_{-}(\lambda \mid \beta+2),
\end{aligned}
$$

and

$$
\begin{aligned}
\mathcal{T}(\lambda) & =K_{+}^{(R)}(\lambda \mid \beta)_{11} \mathcal{A}_{-}(\lambda \mid \beta+2)+K_{+}^{(R)}(\lambda \mid \beta)_{21} \mathcal{B}_{-}(\lambda \mid \beta+2) \\
& +K_{+}^{(R)}(\lambda \mid \beta)_{12} \mathcal{C}_{-}(\lambda \mid \beta+2)+K_{+}^{(R)}(\lambda \mid \beta)_{22} \mathcal{D}_{-}(\lambda \mid \beta+2) .
\end{aligned}
$$

Proof. To prove the two decompositions of the transfer matrix we first remark that the following identities hold:

$$
\left(\begin{array}{c}
\tilde{Y}_{\beta-1}(\lambda) \\
\tilde{X}_{\beta+3}(\lambda)
\end{array}\right)\left(\begin{array}{ll}
\hat{X}_{\beta+3}(\lambda) & \hat{Y}_{\beta-1}(\lambda)
\end{array}\right)=\frac{\left(\begin{array}{cc}
1 & 0 \\
0 & 1
\end{array}\right)}{\theta(\lambda+(\alpha+1) \eta) \theta_{4}(2 \lambda \mid 2 \omega)},
$$

and

$$
\hat{X}_{\beta+3}(\lambda) \bar{Y}_{\beta-1}(\lambda)+\hat{Y}_{\beta-1}(\lambda) \bar{X}_{\beta+3}(\lambda)=\frac{\left(\begin{array}{cc}
1 & 0 \\
0 & 1
\end{array}\right)}{\theta(\lambda+(\alpha+1) \eta) \theta_{4}(2 \lambda \mid 2 \omega)} .
$$


The formulae (3.9) and (3.54) imply the following chain of identities:

$$
\begin{aligned}
& \mathcal{A}_{-}(\lambda \mid \beta+2) K_{+}^{(L)}(\lambda \mid \beta)_{11}+\mathcal{B}_{-}(\lambda \mid \beta) K_{+}^{(L)}(\lambda \mid \beta)_{21}+\mathcal{D}_{-}(\lambda \mid \beta+2) K_{+}^{(L)}(\lambda \mid \beta)_{22}+\mathcal{C}_{-}(\lambda \mid \beta+4) K_{+}^{(L)}(\lambda \mid \beta)_{12} \\
& =\frac{\tilde{Y}_{\beta-1}(\lambda-\eta / 2) \mathcal{U}_{-}(\lambda) K_{+}(\lambda) \hat{X}_{\beta+3}(\lambda-\eta / 2)+\tilde{X}_{\beta+3}(\lambda-\eta / 2) \mathcal{U}_{-}(\lambda) K_{+}(\lambda) \hat{Y}_{\beta-1}(\lambda-\eta / 2)}{\left(\theta(\lambda+(\alpha+1 / 2) \eta) \theta_{4}(2 \lambda-\eta \mid 2 \omega)\right)^{-1}} \\
& =\frac{\operatorname{tr}_{0}\left\{( \begin{array} { c } 
{ \tilde { Y } _ { \beta - 1 } ( \lambda - \eta / 2 ) } \\
{ \tilde { X } _ { \beta + 3 } ( \lambda - \eta / 2 ) }
\end{array} ) \mathcal { U } _ { - } ( \lambda ) K _ { + } ( \lambda ) \left(\hat{X}_{\beta+3}(\lambda-\eta / 2)\right.\right.}{\left.\left.\hat{Y}_{\beta-1}(\lambda-\eta / 2)\right)\right\}} \\
& =\frac{\operatorname{tr}_{0}\left\{\left(\begin{array}{cc}
\hat{X}_{\beta+3}(\lambda-\eta / 2) & \hat{Y}_{\beta-1}(\lambda-\eta / 2)
\end{array}\right)\left(\begin{array}{c}
\tilde{Y}_{\beta-1}(\lambda-\eta / 2) \\
\tilde{X}_{\beta+3}(\lambda-\eta / 2)
\end{array}\right) \mathcal{U}_{-}(\lambda) K_{+}(\lambda)\right\}}{\left(\theta(\lambda+(\alpha+1 / 2) \eta) \theta_{4}(2 \lambda-\eta \mid 2 \omega)\right)^{-1}} \\
& =\operatorname{tr}_{0}\left\{\mathcal{U}_{-}(\lambda) K_{+}(\lambda)\right\}=\mathcal{T}(\lambda) \text {. }
\end{aligned}
$$

Similarly, the formulae (3.9) and (3.8) imply the following chain of identities:

$$
\begin{aligned}
& K_{+}^{(R)}(\lambda \mid \beta)_{11} \mathcal{A}_{-}(\lambda \mid \beta+2)+K_{+}^{(R)}(\lambda \mid \beta)_{12} \mathcal{C}_{-}(\lambda \mid \beta+2)+K_{+}^{(R)}(\lambda \mid \beta)_{22} \mathcal{D}_{-}(\lambda \mid \beta+2)+K_{+}^{(R)}(\lambda \mid \beta)_{21} \mathcal{B}_{-}(\lambda \mid \beta+2) \\
& =\bar{Y}_{\beta+1}(\eta / 2-\lambda) K_{+}(\lambda) \mathcal{U}_{-}(\lambda) X_{\beta+1}(\eta / 2-\lambda)+\bar{X}_{\beta+1}(\eta / 2-\lambda) \mathcal{U}_{-}(\lambda) K_{+}(\lambda) Y_{\beta+1}(\eta / 2-\lambda) \\
& =\operatorname{tr}_{0}\left\{\left(\begin{array}{c}
\bar{Y}_{\beta+1}(\eta / 2-\lambda) \\
\bar{X}_{\beta+1}(\eta / 2-\lambda)
\end{array}\right) K_{+}(\lambda) \mathcal{U}_{-}(\lambda)\left(\begin{array}{cc}
X_{\beta+1}(\eta / 2-\lambda) & Y_{\beta+1}(\eta / 2-\lambda)
\end{array}\right)\right\} \\
& =\operatorname{tr}_{0}\left\{\left(\begin{array}{cc}
X_{\beta+1}(\eta / 2-\lambda) & Y_{\beta+1}(\eta / 2-\lambda)
\end{array}\right)\left(\begin{array}{c}
\bar{Y}_{\beta+1}(\eta / 2-\lambda) \\
\bar{X}_{\beta+1}(\eta / 2-\lambda)
\end{array}\right) K_{+}(\lambda) \mathcal{U}_{-}(\lambda)\right\} \\
& =\operatorname{tr}_{0}\left\{K_{+}(\lambda) \mathcal{U}_{-}(\lambda)\right\}=\mathcal{T}(\lambda) .
\end{aligned}
$$

Proposition 3.3. The following two explicitly even in $\lambda$ representations of the transfer matrix hold:

$$
\begin{aligned}
& \mathcal{T}(\lambda)=\mathrm{a}_{+}(\lambda) \mathcal{A}_{-}(\lambda \mid \beta+2)+\mathrm{a}_{+}(-\lambda) \mathcal{A}_{-}(-\lambda \mid \beta+2)+K_{+}^{(L)}(\lambda \mid \beta)_{12} \mathcal{C}_{-}(\lambda \mid \beta+4)+K_{+}^{(L)}(\lambda \mid \beta)_{21} \mathcal{B}_{-}(\lambda \mid \beta) \\
& \mathcal{T}(\lambda)=\mathrm{d}_{+}(\lambda) \mathcal{D}_{-}(\lambda \mid \beta+2)+\mathrm{d}_{+}(-\lambda) \mathcal{D}_{-}(-\lambda \mid \beta+2)+K_{+}^{(R)}(\lambda \mid \beta)_{12} \mathcal{C}_{-}(\lambda \mid \beta+2)+K_{+}^{(R)}(\lambda \mid \beta)_{21} \mathcal{B}_{-}(\lambda \mid \beta+2)
\end{aligned}
$$

where we have defined:

$$
\mathrm{a}_{+}(\lambda)=\frac{\theta(2 \lambda+\eta) \theta((\beta+1) \eta)}{\theta(2 \lambda) \theta((\beta+2) \eta)} K_{+}^{(L)}(-\lambda \mid \beta)_{22}, \mathrm{~d}_{+}(\lambda)=\frac{\theta(2 \lambda+\eta) \theta((\beta+1) \eta)}{\theta(2 \lambda) \theta(\beta \eta)} K_{+}^{(R)}(-\lambda \mid \beta)_{11} .
$$

Proof. The decompositions of the transfer matrix given in the previous proposition can be rewritten in the following 
way:

$$
\begin{aligned}
\mathcal{T}(\lambda) & =\left(K_{+}^{(L)}(\lambda \mid \beta)_{11}+\frac{\theta(\eta) \theta(2 \lambda+(\beta+1) \eta)}{\theta(2 \lambda) \theta((\beta+2) \eta)} K_{+}^{(L)}(\lambda \mid \beta)_{22}\right) \mathcal{A}_{-}(\lambda \mid \beta+2)+\mathcal{A}_{-}(-\lambda \mid \beta+2) \\
& \times\left(\frac{\theta(2 \lambda-\eta) \theta((\beta+1) \eta)}{\theta(2 \lambda) \theta((\beta+2) \eta)} K_{+}^{(L)}(\lambda \mid \beta)_{22}\right)+K_{+}^{(L)}(\lambda \mid \beta)_{21} \mathcal{B}_{-}(\lambda \mid \beta)+K_{+}^{(L)}(\lambda \mid \beta)_{12} \mathcal{C}_{-}(\lambda \mid \beta+4), \quad(3.61) \\
\mathcal{T}(\lambda) & =\left(K_{+}^{(R)}(\lambda \mid \beta)_{22}-\frac{\theta(\eta) \theta(2 \lambda-(\beta+1) \eta)}{\theta(2 \lambda) \theta(\beta \eta)} K_{+}^{(R)}(\lambda \mid \beta)_{11}\right) \mathcal{D}_{-}(\lambda \mid \beta+2)+\mathcal{D}_{-}(-\lambda \mid \beta+2) \\
& \times\left(\frac{\theta(2 \lambda-\eta) \theta((\beta+1) \eta)}{\theta(2 \lambda) \theta(\beta \eta)} K_{+}^{(R)}(\lambda \mid \beta)_{11}\right)+K_{+}^{(R)}(\lambda \mid \beta)_{21} \mathcal{B}_{-}(\lambda \mid \beta+2)+K_{+}^{(R)}(\lambda \mid \beta)_{12} \mathcal{C}_{-}(\lambda \mid \beta+2),
\end{aligned}
$$

once we use the properties (3.25)-(3.26). Then the identities:

$$
\begin{aligned}
& K_{+}^{(L)}(\lambda \mid \beta)_{11}+\frac{\theta(\eta) \theta(2 \lambda+(\beta+1) \eta)}{\theta(2 \lambda) \theta((\beta+2) \eta)} K_{+}^{(L)}(\lambda \mid \beta)_{22}=\frac{\theta(2 \lambda+\eta) \theta((\beta+1) \eta)}{\theta(2 \lambda) \theta((\beta+2) \eta)} K_{+}^{(L)}(-\lambda \mid \beta)_{22} \\
& K_{+}^{(R)}(\lambda \mid \beta)_{22}-\frac{\theta(\eta) \theta(2 \lambda-(\beta+1) \eta)}{\theta(2 \lambda) \theta(\beta \eta)} K_{+}^{(R)}(\lambda \mid \beta)_{11}=\frac{\theta(2 \lambda+\eta) \theta((\beta+1) \eta)}{\theta(2 \lambda) \theta(\beta \eta)} K_{+}^{(R)}(-\lambda \mid \beta)_{11}
\end{aligned}
$$

that one can verify by direct computations, imply the announced results.

The functions $a_{+}(\lambda)$ and $d_{+}(\lambda)$ will be crucial in the SOV description of the transfer matrix spectrum and so will be the following properties:

Lemma 3.3. Using the freedom in the choice of the gauge parameters to fix:

$$
K_{+}^{(L)}(\lambda \mid \beta)_{12}=0
$$

keeping completely arbitrary the six boundary parameters, the following quantum determinant conditions are satisfied:

$$
\begin{aligned}
\frac{\operatorname{det}_{q} K_{+}(\lambda) p(\lambda-\eta / 2)}{\theta(\eta-2 \lambda) \theta(2 \lambda+\eta) r(\lambda+\eta / 2) r(-\lambda+\eta / 2)} & =\mathbf{a}_{+}(\lambda+\eta / 2) \mathbf{a}_{+}(-\lambda+\eta / 2) \\
& =\mathbf{d}_{+}(\lambda+\eta / 2) \mathbf{d}_{+}(-\lambda+\eta / 2),
\end{aligned}
$$

where:

$$
\operatorname{det}_{q} K_{+}(\lambda)=p(-\lambda-\eta / 2) g_{+}(\lambda+\eta / 2) g_{+}(-\lambda+\eta / 2) \text {. }
$$

Proof. Let us prove only the identity (3.66) as the other one follows similarly. From the very definitions of these functions it holds:

$$
\begin{aligned}
\mathrm{a}_{+}(\lambda+\eta / 2) \mathrm{a}_{+}(\eta / 2-\lambda) & =\frac{\tilde{X}_{\beta+3}(\eta+\lambda) K_{+}(-\lambda-\eta / 2) Y_{\beta+1}(\eta-\lambda) \tilde{X}_{\beta+3}(\eta-\lambda) K_{+}(\lambda-\eta / 2) Y_{\beta+1}(\lambda+\eta)}{r(\lambda+\eta / 2) r(-\lambda+\eta / 2) \theta(\eta-2 \lambda) \theta(2 \lambda+\eta)(p(-\lambda-\eta / 2) p(\lambda-\eta / 2))^{-1}} \\
& =\frac{\tilde{X}_{\beta+3}(\eta+\lambda) K_{+}(-\lambda-\eta / 2) K_{+}(\lambda-\eta / 2) Y_{\beta+1}(\lambda+\eta)}{r(\lambda+\eta / 2) r(-\lambda+\eta / 2) \theta(\eta-2 \lambda) \theta(2 \lambda+\eta)(p(-\lambda-\eta / 2) p(\lambda-\eta / 2))^{-1}} \\
& =\frac{\operatorname{det}_{q} K_{+}(\lambda) p(\lambda-\eta / 2) \tilde{X}_{\beta+3}(\eta+\lambda) Y_{\beta+1}(\lambda+\eta)}{\theta(\eta-2 \lambda) \theta(2 \lambda+\eta) r(\lambda+\eta / 2) r(-\lambda+\eta / 2)} \\
& =\frac{\operatorname{det}_{q} K_{+}(\lambda) p(\lambda-\eta / 2)}{\theta(\eta-2 \lambda) \theta(2 \lambda+\eta) r(\lambda+\eta / 2) r(-\lambda+\eta / 2)}
\end{aligned}
$$


The second line is obtained by using the identity (3.9) once we add to the first line the following term:

$$
\frac{\tilde{X}_{\beta+3}(\eta+\lambda) K_{+}(-\lambda-\eta / 2) X_{\beta+3}(-\lambda+\eta) \tilde{Y}_{\beta+1}(-\lambda+\eta) K_{+}(\lambda-\eta / 2) Y_{\beta+1}(\lambda+\eta)}{r(\lambda+\eta / 2) r(-\lambda+\eta / 2) \theta(\eta-2 \lambda) \theta(2 \lambda+\eta)(p(-\lambda-\eta / 2) p(\lambda-\eta / 2))^{-1}},
$$

which is zero being:

$$
\tilde{Y}_{\beta+1}(-\lambda+\eta) K_{+}(\lambda-\eta / 2) Y_{\beta+1}(\lambda+\eta)=0,
$$

for the condition (3.65). Then the third line follows as by dirtect computation one can prove:

$$
\frac{\operatorname{det}_{q} K_{+}(\lambda)}{p(-\lambda-\eta / 2)}=K_{+}(\lambda-\eta / 2) K_{+}(-\lambda-\eta / 2)
$$

and the last identity is once again due to (3.9).

\section{SOV representations}

Let us introduced the following gauge transformed matrices starting from the $K_{-}(\lambda)$ boundary matrix:

$$
\begin{aligned}
& K_{-}(\lambda \mid \beta)_{11} \equiv \tilde{Y}_{\beta+\mathrm{N}-1}(\lambda-\eta / 2) K_{-}(\lambda) X_{\beta+\mathrm{N}-1}(\eta / 2-\lambda), \\
& K_{-}(\lambda \mid \beta)_{12} \equiv \tilde{Y}_{\beta+\mathrm{N}-1}(\lambda-\eta / 2) K_{-}(\lambda) Y_{\beta+\mathrm{N}-1}(\eta / 2-\lambda), \\
& K_{-}(\lambda \mid \beta)_{21} \equiv \tilde{X}_{\beta+\mathrm{N}+1}(\lambda-\eta / 2) K_{-}(\lambda) X_{\beta+\mathrm{N}-1}(\eta / 2-\lambda), \\
& K_{-}(\lambda \mid \beta)_{22} \equiv \tilde{X}_{\beta+\mathrm{N}+1}(\lambda-\eta / 2) K_{-}(\lambda) Y_{\beta+\mathrm{N}-1}(\eta / 2-\lambda),
\end{aligned}
$$

and

$$
\begin{aligned}
\tilde{K}_{-}(\lambda \mid \beta)_{11} & \equiv \tilde{Y}_{\beta+\mathrm{N}-3}(\lambda-\eta / 2) K_{-}(\lambda) X_{\beta+\mathrm{N}-1}(\eta / 2-\lambda), \\
\tilde{K}_{-}(\lambda \mid \beta)_{12} & \equiv \tilde{Y}_{\beta+\mathrm{N}-3}(\lambda-\eta / 2) K_{-}(\lambda) Y_{\beta+\mathrm{N}-1}(\eta / 2-\lambda), \\
\tilde{K}_{-}(\lambda \mid \beta)_{21} & \equiv \tilde{X}_{\beta+\mathrm{N}-1}(\lambda-\eta / 2) K_{-}(\lambda) X_{\beta+\mathrm{N}-1}(\eta / 2-\lambda), \\
\tilde{K}_{-}(\lambda \mid \beta)_{22} & \equiv \tilde{X}_{\beta+\mathrm{N}-1}(\lambda-\eta / 2) K_{-}(\lambda) Y_{\beta+\mathrm{N}-1}(\eta / 2-\lambda),
\end{aligned}
$$

then the following theorem holds:

Theorem 4.1. Let the following conditions be satisfied:

$$
\xi_{a} \neq \xi_{b}+\operatorname{rr} \bmod (\pi, \pi \omega) \forall a \neq b \in\{1, \ldots, \mathrm{N}\} \text { and } r \in\{-1,0,1\}
$$

then:

1b) for all the gauge parameters $\alpha, \beta \in \mathbb{C}$ such that:

$$
K_{-}(\lambda \mid \beta)_{12} \neq 0
$$

$\mathcal{B}_{-}(\lambda \mid \beta)$ is left pseudo-diagonalizable and with simple pseudo-spectrum.

$\left.2_{b}\right)$ for all the gauge parameters $\alpha, \beta \in \mathbb{C}$ such that:

$$
\tilde{K}_{-}(\lambda \mid-\beta)_{21} \neq 0,
$$

$\mathcal{B}_{-}(\lambda \mid \beta+2)$ is right pseudo-diagonalizable and with simple pseudo-spectrum. 
1) for all the gauge parameters $\alpha, \beta \in \mathbb{C}$ such that:

$$
K_{-}(\lambda \mid-\beta-2)_{12} \neq 0,
$$

$\mathcal{C}_{-}(\lambda \mid \beta+4)$ is left pseudo-diagonalizable and with simple pseudo-spectrum.

2) for all the gauge parameters $\alpha, \beta \in \mathbb{C}$ such that:

$$
\tilde{K}_{-}(\lambda \mid \beta+2)_{21} \neq 0
$$

$\mathcal{C}_{-}(\lambda \mid \beta+2)$ is right pseudo-diagonalizable and with simple pseudo-spectrum.

In the next sections we will show the theorem and clarify the terminology by an explicit construction in the cases $1_{b}$ ) and $2_{b}$ ). Note that the construction in the cases $1_{c}$ ) and $2_{c}$ ) can be induced from the cases $1_{b}$ ) and $2_{b}$ ) thanks to the $\beta$-symmetries (3.44).

\subsection{Gauge transformed reflection algebra in $\mathcal{B}_{-}(\mid \beta)-\mathrm{SOV}$ representations}

\subsubsection{Simultaneous $B(\lambda \mid \beta)$ and $\bar{B}(\lambda \mid \beta)$ bulk left reference state}

Let us define the following state:

$$
\langle\beta| \equiv N_{\beta} \otimes_{n=1}^{\mathrm{N}} \tilde{Y}_{\beta+\mathrm{N}-n}^{(n)}\left(\xi_{n}\right), \quad N_{\beta}=2^{\mathrm{N}} \prod_{n=1}^{\mathrm{N}} \theta(\mathrm{N}-n+\beta) \eta
$$

where $\tilde{Y}_{\beta+\mathrm{N}-n}^{(n)}\left(\xi_{n}\right)$ is the covector $\tilde{Y}_{\beta+\mathrm{N}-n}\left(\xi_{n}\right)$ in the local $\mathrm{L}_{n}$ quantum covector space and $N_{\beta}$ is a normalization factor.

Proposition 4.1. The state $\langle\beta|$ is a simultaneous $\bar{B}(\lambda \mid \beta)$ and $B(\lambda \mid \beta)$ eigenstate associated to the eigenvalue zero, for which the following identities hold:

$$
\begin{aligned}
\langle\beta| B(\lambda \mid \beta) & =\langle\beta| \bar{B}(\lambda \mid \beta)=\underline{0}, \\
\langle\beta| A(\lambda \mid \beta) & =\frac{\theta((\mathrm{N}+\beta) \eta)}{\theta(\beta \eta)} \prod_{n=1}^{\mathrm{N}} \theta\left(\lambda-\xi_{n}+\eta / 2\right)\langle\beta-1| \\
\langle\beta| D(\lambda \mid \beta) & =\prod_{n=1}^{\mathrm{N}} \theta\left(\lambda-\xi_{n}-\eta / 2\right)\langle\beta+1| \\
\langle\beta| \bar{A}(\lambda \mid \beta) & =\frac{\theta(\beta \eta)}{\theta((\mathrm{N}+\beta) \eta)} \prod_{n=1}^{\mathrm{N}} \theta\left(\lambda+\xi_{n}+\eta / 2\right)\langle\beta+1| \\
\langle\beta| \bar{D}(\lambda \mid \beta) & =\prod_{n=1}^{\mathrm{N}} \theta\left(\lambda+\xi_{n}-\eta / 2\right)\langle\beta-1|
\end{aligned}
$$

Proof. The proposition is a consequence of the following identities for local operators:

$$
\begin{aligned}
& \tilde{Y}_{s}^{(n)}\left(\xi_{n}\right) \tilde{G}_{s}^{-1}(\lambda-\eta / 2) R_{0 n}\left(\lambda-\xi_{n}-\eta / 2\right) \tilde{G}_{s+1}(\lambda-\eta / 2) \\
& =\left(\begin{array}{ll}
\frac{\theta((s+1+\beta) \eta) \theta\left(\lambda-\xi_{n}+\eta / 2\right)}{\theta((s+\beta) \eta)} \tilde{Y}_{s-1}^{(n)}\left(\xi_{n}\right) & \underline{0}_{(n)} \\
* & \theta\left(\lambda-\xi_{n}-\eta / 2\right) \tilde{Y}_{s+1}^{(n)}\left(\xi_{n}\right)
\end{array}\right)
\end{aligned}
$$


where we have used:

$$
\begin{aligned}
& \tilde{Y}_{s}^{(n)}\left(\xi_{n}\right) \tilde{Y}_{s-1}^{(0)}(\lambda-\eta / 2) R_{0 n}\left(\lambda-\xi_{n}-\eta / 2\right) X_{s+2}^{(0)}(\lambda-\eta / 2)=\frac{\theta((s+1+\beta) \eta) \theta\left(\lambda-\xi_{n}+\eta / 2\right)}{\theta((s+\beta) \eta)} \tilde{Y}_{s-1}^{(n)}\left(\xi_{n}\right) \\
& \tilde{Y}_{s}^{(n)}\left(\xi_{n}\right) \tilde{Y}_{s-1}^{(0)}(\lambda-\eta / 2) R_{0 n}\left(\lambda-\xi_{n}-\eta / 2\right) Y_{s}^{(0)}(\lambda-\eta / 2)=\underline{0}_{(n)} \\
& \tilde{Y}_{s}^{(n)}\left(\xi_{n}\right) \tilde{X}_{s+1}^{(0)}(\lambda-\eta / 2) R_{0 n}\left(\lambda-\xi_{n}-\eta / 2\right) Y_{s}^{(0)}(\lambda-\eta / 2)=\theta\left(\lambda-\xi_{n}-\eta / 2\right) \tilde{Y}_{s+1}^{(n)}\left(\xi_{n}\right)
\end{aligned}
$$

and similarly:

$$
\begin{aligned}
& -\tilde{Y}_{s}^{(n)}\left(\xi_{n}\right) \bar{G}_{s+1}^{-1}(\eta / 2-\lambda) \sigma_{0}^{y} R_{0 n}^{t_{0}}\left(-\lambda-\xi_{n}-\eta / 2\right) \sigma_{0}^{y} \bar{G}_{s}(\eta / 2-\lambda) \\
& =\tilde{Y}_{s}^{(n)}\left(\xi_{n}\right) \bar{G}_{s+1}^{-1}(\eta / 2-\lambda) R_{n 0}\left(\lambda+\xi_{n}-\eta / 2\right) \bar{G}_{s}(\eta / 2-\lambda) \\
& =\left(\begin{array}{ll}
\frac{\theta((s+\beta) \eta) \theta\left(\lambda+\xi_{n}+\eta / 2\right)}{\theta((s+1+\beta) \eta)} \tilde{Y}_{s+1}^{(n)}\left(\xi_{n}\right) & \underline{0}_{(n)} \\
* & \theta\left(\lambda+\xi_{n}-\eta / 2\right) \tilde{Y}_{s-1}^{(n)}\left(\xi_{n}\right)
\end{array}\right) .
\end{aligned}
$$

\subsubsection{Simultaneous $C(\lambda \mid \beta)$ and $\bar{C}(\lambda \mid \beta)$ bulk right reference state}

Let us define the following state:

$$
|\beta+1\rangle \equiv \otimes_{n=1}^{\mathrm{N}} X_{\beta+\mathrm{N}-n+1}^{(n)}\left(\xi_{n}\right),
$$

where $X_{\beta+\mathrm{N}-n}^{(n)}\left(\xi_{n}\right)$ is the vector $X_{\beta+\mathrm{N}-n}\left(\xi_{n}\right)$ in the local $\mathrm{R}_{n}$ quantum space. Then the following proposition holds:

Proposition 4.2. The state $|\beta+1\rangle$ is a simultaneous $\bar{C}(\lambda \mid \beta)$ and $C(\lambda \mid \beta)$ right eigenstate associated to the eigenvalue zero and the following identities hold:

$$
\begin{aligned}
C(\lambda \mid \beta)|\beta+1\rangle & =\bar{C}(\lambda \mid \beta)|\beta+1\rangle=\underline{0}, \\
A(\lambda \mid \beta)|\beta+1\rangle & =\prod_{n=1}^{\mathrm{N}} \theta\left(\lambda-\xi_{n}+\eta / 2\right)|\beta+2\rangle, \\
D(\lambda \mid \beta)|\beta+1\rangle & =\frac{\theta(\eta(\mathrm{N}+\beta))}{\theta(\eta \beta)} \prod_{n=1}^{\mathrm{N}} \theta\left(\lambda-\xi_{n}-\eta / 2\right)|\beta\rangle, \\
\bar{A}(\lambda \mid \beta)|\beta+1\rangle & =\prod_{n=1}^{\mathrm{N}} \theta\left(\lambda+\xi_{n}+\eta / 2\right)|\beta\rangle, \\
\bar{D}(\lambda \mid \beta)|\beta+1\rangle & =\frac{\theta(\eta \beta)}{\theta(\eta(\mathrm{N}+\beta))} \prod_{n=1}^{\mathrm{N}} \theta\left(\lambda+\xi_{n}-\eta / 2\right)|\beta+2\rangle .
\end{aligned}
$$

\subsubsection{Gauge transformed reflection algebra in left $\mathcal{B}_{-}(\mid \beta)$-SOV representations}

The left $\mathcal{B}_{-}(\mid \beta)$-pseudo-eigenbasis is here constructed and the representation of the gauge transformed boundary operator $\mathcal{A}_{-}(\lambda \mid \beta)$ in this basis is determined. In the following we will need of the following notations:

$$
\zeta_{-1} \equiv \eta / 2, \quad \zeta_{-2} \equiv(\eta-\pi) / 2, \quad \zeta_{-3} \equiv(\eta-\pi \omega) / 2, \quad \zeta_{-4} \equiv(\eta-\pi-\pi \omega) / 2,
$$


$\zeta_{-a-4} \equiv \zeta_{-a}+\pi \omega$ for $a \in\{1,2,3,4\}$ and also

$$
\begin{aligned}
\zeta_{n}^{\left(h_{n}\right)} & \equiv \varphi_{n}\left[\xi_{n}+\left(h_{n}-\frac{1}{2}\right) \eta\right] \forall n \in\{1, \ldots, 2 \mathrm{~N}\}, h_{n} \in\{0,1\} \text { with } h_{\mathrm{N}+n} \equiv h_{n} \forall n \in\{1, \ldots, \mathrm{N}\} \\
\varphi_{a} & \equiv 1-2 z(a-\mathrm{N}) \quad \text { with } z(x)=\{0 \text { for } x \leq 0,1 \text { for } x>0\}
\end{aligned}
$$

Morever, we define the states:

$$
\left\langle\beta, h_{1}, \ldots, h_{\mathrm{N}}\right| \equiv \frac{1}{\mathrm{~N}_{\beta+2}}\langle\beta| \prod_{n=1}^{\mathrm{N}}\left(\frac{\mathcal{A}_{-}\left(\eta / 2-\xi_{n} \mid \beta+2\right)}{\mathrm{A}_{-}\left(\eta / 2-\xi_{n}\right)}\right)^{h_{n}}
$$

where, at this stage, $\mathbf{N}_{\beta+2}$ is just an arbitrary normalization function of $\beta$ and $\langle\beta|$ is the reference state defined in (4.14). It is important pointing out that the states $\langle\beta, \mathbf{h}|$ are well defined states; i.e. their definition does not depend on the order of operator $\mathcal{A}_{-}\left(-\zeta_{b}^{(0)} \mid \beta+2\right)$ as one can verify directly from the commutation relations (3.43).

Theorem 4.2. Left $\mathcal{B}_{-}(\mid \beta)-S O V$-representations Let us assume that (4.9) and (4.10) are satisfied, then the states (4.36) define a basis formed out of pseudo-eigenstates of $\mathcal{B}_{-}(\lambda \mid \beta)$ :

$$
\langle\beta, \boldsymbol{h}| \mathcal{B}_{-}(\lambda \mid \beta)=\mathbf{B}_{\beta, \boldsymbol{h}}(\lambda)\langle\beta-2, \boldsymbol{h}|,
$$

where $\langle\beta, \boldsymbol{h}| \equiv\left\langle\beta, h_{1}, \ldots, h_{\mathrm{N}}\right|$ for $\boldsymbol{h} \equiv\left(h_{1}, \ldots, h_{\mathrm{N}}\right)$ and

$$
\mathrm{B}_{\beta, \boldsymbol{h}}(\lambda) \equiv \frac{\mathrm{N}_{\beta}}{\mathrm{N}_{\beta+2}}(-1)^{\mathrm{N}} \theta_{4}(2 \lambda-\eta \mid 2 \omega) \theta(\lambda+(\alpha+1 / 2) \eta) K_{-}(\lambda \mid \beta)_{12} a_{\boldsymbol{h}}(\lambda) a_{\boldsymbol{h}}(-\lambda),
$$

with

$$
a_{\boldsymbol{h}}(\lambda) \equiv \prod_{n=1}^{\mathrm{N}} \theta\left(\lambda-\xi_{n}-\left(h_{n}-\frac{1}{2}\right) \eta\right) .
$$

Moreover, $\mathcal{B}_{-}(\lambda \mid \beta)$ is an order $4 \mathrm{~N}+8$ elliptic polynomials of periods $\pi$ and $2 \pi \omega$ :

$$
\mathcal{B}_{-}(\lambda+\pi \mid \beta)=\mathcal{B}_{-}(\lambda \mid \beta), \mathcal{B}_{-}(\lambda+2 \pi \omega \mid \beta)=\left(e^{-2 i(\lambda-\eta / 2)} / q^{2}\right)^{4 \mathrm{~N}+8} \mathcal{B}_{-}(\lambda \mid \beta),
$$

where $q \equiv e^{i \pi \omega} \cdot \mathcal{A}_{-}(\lambda \mid \beta)$ is an order $4 \mathrm{~N}+8$ elliptic polynomials of periods $\pi$ and $2 \pi \omega$ :

$$
\begin{aligned}
\mathcal{A}_{-}(\lambda+2 \pi \omega \mid \beta) & =\left(-e^{-2 i \lambda} / q^{2}\right)^{4 \mathrm{~N}+8} e^{2 i \alpha_{\mathcal{A}_{-}(\beta)} \mathcal{A}_{-}(\lambda \mid \beta),} \\
\mathcal{A}_{-}(\lambda+\pi \mid \beta) & =\mathcal{A}_{-}(\lambda \mid \beta), \text { where } \alpha_{\mathcal{A}_{-}(\beta)} \equiv 2(\mathrm{~N}+\beta) \eta
\end{aligned}
$$

Moreover, defined the operator $\mathcal{A}_{-}^{(0)}(\lambda \mid \beta+2)$ by the following action on the generic state $\langle\beta, \boldsymbol{h}|$ :

$$
\begin{aligned}
\langle\beta, \boldsymbol{h}| \mathcal{A}_{-}^{(0)}(\lambda \mid \beta+2) & \equiv \sum_{a=1}^{8} \frac{\theta_{1}\left(2(\mathrm{~N}+\beta+2)-\lambda-\sum_{b=1, b \neq a}^{8} \zeta_{-b} \mid 2 \omega\right)}{\theta_{1}\left(2(\mathrm{~N}+\beta+2)-\sum_{b=1}^{8} \zeta_{-b} \mid 2 \omega\right)} \frac{a_{\boldsymbol{h}}(\lambda) a_{\boldsymbol{h}}(-\lambda)}{a_{\boldsymbol{h}}\left(\zeta_{-a}\right) a_{\boldsymbol{h}}\left(-\zeta_{-a}\right)} \\
& \times \prod_{b=1, b \neq a}^{8} \frac{\theta_{1}\left(\lambda-\zeta_{-b} \mid 2 \omega\right)}{\theta_{1}\left(\zeta_{-a}-\zeta_{-b} \mid 2 \omega\right)}\langle\beta, \boldsymbol{h}| \mathcal{A}_{-}\left(\zeta_{-a} \mid \beta+2\right),
\end{aligned}
$$

then the operator:

$$
\widetilde{\mathcal{A}}_{-}(\lambda \mid \beta+2) \equiv \mathcal{A}_{-}(\lambda \mid \beta+2)-\mathcal{A}_{-}^{(0)}(\lambda \mid \beta+2),
$$


has the following action on the generic state $\langle\beta, \boldsymbol{h}|$ :

$$
\begin{aligned}
\langle\beta, \boldsymbol{h}| \widetilde{\mathcal{A}}_{-}(\lambda \mid \beta+2) & =\sum_{a=1}^{2 \mathrm{~N}} \frac{\theta_{4}(2 \lambda-\eta \mid 2 \omega) \theta_{1}(2 \lambda-\eta \mid 2 \omega) \theta_{1}\left(2(\mathrm{~N}+\beta+2)+\zeta_{a}^{\left(h_{a}\right)}-\lambda-\sum_{b=1}^{8} \zeta_{-b} \mid 2 \omega\right)}{\theta_{4}\left(2 \zeta_{a}^{\left(h_{a}\right)}-\eta \mid 2 \omega\right) \theta_{1}\left(2 \zeta_{a}^{\left(h_{a}\right)}-\eta \mid 2 \omega\right) \theta_{1}\left(2(\mathrm{~N}+\beta+2)-\sum_{b=1}^{8} \zeta_{-b} \mid 2 \omega\right)} \\
& \times \frac{\theta_{1}\left(\lambda+\zeta_{a}^{\left(h_{a}\right)} \mid 2 \omega\right) \theta_{2}^{2 \mathrm{~N}}(\lambda \mid 2 \omega)}{\theta_{1}\left(2 \zeta_{a}^{\left(h_{a}\right)} \mid 2 \omega\right) \theta_{2}^{2 \mathrm{~N}}\left(\zeta_{a}^{\left(h_{a}\right)} \mid 2 \omega\right)} \prod_{\substack{b=1 \\
b \neq a \bmod }}^{\mathrm{N}} \frac{\frac{\theta_{4}^{2}(\lambda \mid 2 \omega)}{\theta_{2}^{2}(\lambda \mid 2 \omega)}-\frac{\theta_{4}^{2}\left(\zeta_{b}^{\left(h_{b}\right)} \mid 2 \omega\right)}{\theta_{2}^{2}\left(\zeta_{b}^{\left(h_{b}\right)} \mid 2 \omega\right)}}{\theta_{2}^{2}\left(\zeta_{a}^{\left(h_{a}\right)} \mid 2 \omega\right)}-\frac{\theta_{a}^{(h)}\left(\zeta_{b}^{\left(h_{b}\right)} \mid 2 \omega\right)}{\theta_{2}^{2}\left(\zeta_{b}^{\left(h_{b}\right)} \mid 2 \omega\right)} \mathrm{A}_{-}\left(\zeta_{a}^{\left(h_{a}\right)}\right)\langle\beta, \boldsymbol{h}| T_{a}^{-\varphi_{a}}
\end{aligned}
$$

and:

$$
\left\langle\beta, h_{1}, \ldots, h_{a}, \ldots, h_{\mathrm{N}}\right| T_{a}^{ \pm}=\left\langle\beta, h_{1}, \ldots, h_{a} \pm 1, \ldots, h_{\mathrm{N}}\right|, \mathrm{A}_{-}(\lambda) \equiv r(\lambda) \widehat{\mathrm{A}}_{-}(\lambda) .
$$

Proof. The following boundary-bulk decomposition:

$$
\begin{aligned}
\frac{\mathcal{B}_{-}(\lambda \mid \beta)}{\theta_{4}(2 \lambda-\eta \mid 2 \omega) \theta(\lambda+(\alpha+1 / 2) \eta)} & =K_{-}(\lambda \mid \beta)_{22} B(\lambda \mid \beta) \bar{D}(\lambda \mid \beta-1)+K_{-}(\lambda \mid \beta)_{11} A(\lambda \mid \beta) \bar{B}(\lambda \mid \beta-1) \\
& +K_{-}(\lambda \mid \beta)_{21} B(\lambda \mid \beta) \bar{B}(\lambda \mid \beta-1)+K_{-}(\lambda \mid \beta)_{12} A(\lambda \mid \beta) \bar{D}(\lambda \mid \beta-1),
\end{aligned}
$$

of the gauge transformed reflection algebra generator $\mathcal{B}_{-}(\lambda \mid \beta)$ in terms of the gauge transformed bulk generators and the formulae (4.15)-(4.19) imply that $\langle\beta|$ is a $\mathcal{B}_{-}(\lambda \mid \beta)$-pseudo-eigenstate:

$$
\langle\beta| \mathcal{B}_{-}(\lambda) \equiv \mathbf{B}_{\beta, \mathbf{0}}(\lambda)\langle\beta-2|,
$$

with non-zero pseudo-eigenvalue:

$$
\mathrm{B}_{\beta, \mathbf{0}}(\lambda)=(-1)^{\mathrm{N}} K_{-}(\lambda \mid \beta)_{12} \frac{\mathrm{N}_{\beta}}{\mathrm{N}_{\beta+2}} \theta_{4}(2 \lambda-\eta \mid 2 \omega) \theta(\lambda+(\alpha+1 / 2) \eta) a_{\mathbf{0}}(\lambda) a_{\mathbf{0}}(-\lambda) .
$$

To prove the validity of (4.37) we can use now step by step the procedure described in [54] starting from the gauge transformed reflection algebra commutation relations. Under the condition (4.9), these relations also imply that the set of states $\langle\beta, \mathbf{h}|$ forms a set of $2^{\mathrm{N}}$ independent states, i.e. a $\mathcal{B}_{-}(\lambda \mid \beta)$-pseudo-eigenbasis of the left representation space. Moreover, the definition of the states $\langle\beta, \mathbf{h}|$ and the commutation relation (3.41) allow to define the action of $\mathcal{A}_{-}\left(\zeta_{b}^{\left(h_{b}\right)} \mid \beta+2\right)$ for $b \in\{1, \ldots, 2 \mathrm{~N}\}$ once we use the quantum determinant relations and the conditions:

$$
\langle\beta| \mathcal{A}_{-}\left(\xi_{n}-\eta / 2 \mid \beta+2\right)=\underline{0}, \quad\langle\beta| \mathcal{A}_{-}\left(\eta / 2-\xi_{n} \mid \beta+2\right) \neq \underline{0}
$$

which trivially follows from the boundary-bulk decomposition:

$$
\begin{aligned}
\frac{\mathcal{A}_{-}(\lambda \mid \beta+2)}{\theta_{4}(2 \lambda-\eta \mid 2 \omega) \theta(\lambda+(\alpha+1 / 2) \eta)} & =\bar{K}_{-}(\lambda \mid \beta)_{11} A(\lambda \mid \beta) \bar{A}(\lambda \mid \beta+1)+\bar{K}_{-}(\lambda \mid \beta)_{22} B(\lambda \mid \beta) \bar{C}(\lambda \mid \beta+1) \\
& +\bar{K}_{-}(\lambda \mid \beta)_{21} B(\lambda \mid \beta) \bar{A}(\lambda \mid \beta+1)+\bar{K}_{-}(\lambda \mid \beta)_{12} A(\lambda \mid \beta) \bar{C}(\lambda \mid \beta+1),
\end{aligned}
$$

where we have defined

$$
\begin{aligned}
\bar{K}_{-}(\lambda \mid \beta)_{11} & \equiv \tilde{Y}_{\beta+\mathrm{N}-1}(\lambda-\eta / 2) K_{-}(\lambda) X_{\beta+\mathrm{N}+1}(\eta / 2-\lambda), \\
\bar{K}_{-}(\lambda \mid \beta)_{12} & \equiv \tilde{Y}_{\beta+\mathrm{N}-1}(\lambda-\eta / 2) K_{-}(\lambda) Y_{\beta+\mathrm{N}+1}(\eta / 2-\lambda), \\
\bar{K}_{-}(\lambda \mid \beta)_{21} & \equiv \tilde{X}_{\beta+\mathrm{N}+1}(\lambda-\eta / 2) K_{-}(\lambda) X_{\beta+\mathrm{N}+1}(\eta / 2-\lambda), \\
\bar{K}_{-}(\lambda \mid \beta)_{22} & \equiv \tilde{X}_{\beta+\mathrm{N}+1}(\lambda-\eta / 2) K_{-}(\lambda) Y_{\beta+\mathrm{N}+1}(\eta / 2-\lambda) .
\end{aligned}
$$


The fact that the operator $\mathcal{B}_{-}(\lambda \mid \beta)$ is an order $4 \mathrm{~N}+8$ elliptic polynomials of periods $\pi$ and $2 \pi \omega$ which satisfies (4.40) can be simply derived from the functional form of its pseudo-eigenvalues once we recall the identities: 8 :

$$
\theta_{a}(x+\pi \mid 2 \omega)=(-1)^{\delta_{a, 1}+\delta_{a, 2}} \theta_{a}(x \mid 2 \omega), \quad \theta_{a}(x+2 \pi \omega \mid 2 \omega)=(-1)^{\delta_{a, 1}+\delta_{a, 4}} e^{-2 i(x+\pi \omega)} \theta_{a}(x \mid 2 \omega),
$$

from which also follows:

$$
\theta(x+\pi)=-\theta(x), \quad \theta(x+2 \pi \omega)=e^{-4 i(x+\pi \omega)} \theta(x) .
$$

The fact that the operator $\mathcal{A}_{-}(\lambda \mid \beta)$ is an order $4 \mathrm{~N}+8$ elliptic polynomials of periods $\pi$ and $2 \pi \omega$ which satisfies (4.41)-(4.42) can be simply derived from (4.40) by using the commutation relations (3.41). Indeed, shifting the variable $\lambda_{2}$ in $\lambda_{2}+2 \pi \omega$ and using the transformation properties (4.40) and (4.54), we get:

$$
\begin{aligned}
& f_{\mathcal{A}_{-}(\beta+2)}\left(\lambda_{2}\right) \mathcal{A}_{-}\left(\lambda_{2} \mid \beta+2\right) \mathcal{B}_{-}\left(\lambda_{1} \mid \beta\right)= \\
& \quad \frac{\theta\left(\lambda_{1}-\lambda_{2}+\eta\right) \theta\left(\lambda_{2}+\lambda_{1}-\eta\right)}{\theta\left(\lambda_{1}-\lambda_{2}\right) \theta\left(\lambda_{1}+\lambda_{2}\right)} e^{8 i \eta} f_{\mathcal{A}_{-}(\beta)}\left(\lambda_{2}\right) \mathcal{B}_{-}\left(\lambda_{1} \mid \beta\right) \mathcal{A}_{-}\left(\lambda_{2} \mid \beta\right) \\
& \quad+\frac{\theta\left(\lambda_{1}+\lambda_{2}-\eta\right) \theta\left(\lambda_{1}-\lambda_{2}+(\beta-1) \eta\right) \theta(\eta)}{\theta\left(\lambda_{2}-\lambda_{1}\right) \theta\left(\lambda_{1}+\lambda_{2}\right) \theta((\beta-1) \eta) e^{-4 i \beta \eta}} f_{\mathcal{B}_{-}(\beta)}\left(\lambda_{2}\right) \mathcal{B}_{-}\left(\lambda_{2} \mid \beta\right) \mathcal{A}_{-}\left(\lambda_{1} \mid \beta\right) \\
& \quad+\frac{\theta(\eta) \theta\left(\lambda_{1}+\lambda_{2}-\beta \eta\right)}{\theta\left(\lambda_{1}+\lambda_{2}\right) \theta((\beta-1) \eta)} e^{4 i \beta \eta} f_{\mathcal{B}_{-}(\beta)}\left(\lambda_{2}\right) \mathcal{B}_{-}\left(\lambda_{2} \mid \beta\right) \mathcal{D}_{-}\left(\lambda_{1} \mid \beta\right)
\end{aligned}
$$

where $f_{\mathcal{A}_{-}(\beta)}(\lambda)$ is defined by:

$$
\mathcal{A}_{-}(\lambda+2 \pi \omega \mid \beta)=f_{\mathcal{A}_{-}(\beta)}(\lambda) \mathcal{A}_{-}(\lambda \mid \beta)
$$

which implies:

$$
f_{\mathcal{A}_{-}(\beta)}(\lambda) \equiv\left(-e^{-2 i \lambda} / q^{2}\right)^{4 \mathrm{~N}+8} e^{2 i \alpha_{\mathcal{A}_{-}(\beta)}} \text { where } \alpha_{\mathcal{A}_{-}(\beta)} \equiv 2(\mathrm{~N}+\beta) \eta .
$$

Moreover, by the definition (4.43) it is simple to argue that the operator $\mathcal{A}_{-}^{(0)}(\lambda \mid \beta)$ is also an order $4 \mathrm{~N}+8$ elliptic polynomial of periods $\pi$ and $2 \pi \omega$ which satisfies (4.41) and (4.42) and then the same is true for $\widetilde{\mathcal{A}}_{-}(\lambda \mid \beta)$. These properties together with the identities:

$$
\widetilde{\mathcal{A}}_{-}\left(\zeta_{-a} \mid \beta\right) \equiv \underline{0} \text { for any } a \in\{1, \ldots, 8\},
$$

imply the interpolation formula (4.45) by using the following interpolation formula:

$$
\mathcal{P}(\lambda)=\sum_{a=1}^{\mathrm{M}} \frac{\theta\left(\alpha_{\mathcal{P}}+x_{a}-\lambda-\sum_{n=1}^{\mathrm{M}} x_{n}\right)}{\theta\left(\alpha_{\mathcal{P}}-\sum_{n=1}^{\mathrm{M}} x_{n}\right)} \prod_{b \neq a} \frac{\theta\left(\lambda-x_{b}\right)}{\theta\left(x_{a}-x_{b}\right)} \mathcal{P}\left(x_{a}\right),
$$

which holds true for any order M elliptic polynomial such that:

$$
\mathcal{P}(\lambda+\pi)=(-1)^{\mathrm{M}} \mathcal{P}(\lambda), \quad \mathcal{P}(\lambda+2 \pi \omega)=\left(-e^{-2 i \lambda} / q^{2}\right)^{\mathrm{M}} e^{2 i \alpha_{\mathcal{P}}} \mathcal{P}(\lambda)
$$

\subsubsection{Gauge transformed reflection algebra in right $\mathcal{B}_{-}(\mid \beta)-S O V$ representations}

The right $\mathcal{B}_{-}(\mid \beta)$-pseudo-eigenbasis is here constructed and the representation of the gauge transformed boundary operator $\mathcal{D}_{-}(\lambda \mid \beta)$ in this basis is determined. Let us use the following notation:

$$
\overline{|\beta\rangle} \equiv|-\beta+2\rangle,
$$

\footnotetext{
${ }^{8}$ See the equations $8.182-1,8.182-3$ and $8.183-5,8.183-6$ at page 878 of $[80$ ].
} 
where $|\beta\rangle$ is the right reference state defined in (4.27). Further, let us introduce the states:

$$
\left|\beta, h_{1}, \ldots, h_{\mathrm{N}}\right\rangle \equiv \frac{1}{\mathrm{~N}_{\beta}} \prod_{n=1}^{\mathrm{N}}\left(\frac{\mathcal{D}_{-}\left(\xi_{n}+\eta / 2 \mid \beta\right)}{k_{n}^{(\beta)} \mathrm{A}_{-}\left(\eta / 2-\xi_{n}\right)}\right)^{\left(1-h_{n}\right)} \overline{|\beta\rangle},
$$

where:

$$
k_{a}^{(\beta)} \equiv \frac{\theta\left(2 \xi_{a}+\eta\right) \theta(\beta \eta) \theta_{1}\left(2(\mathrm{~N}+2-\beta)-\sum_{b=1}^{8} \zeta_{-b}-2 \xi_{a} \mid 2 \omega\right) \theta_{1}(\eta \mid 2 \omega) \theta_{2}^{2 \mathrm{~N}}\left(\zeta_{a}^{(1)} \mid 2 \omega\right)}{\theta(\eta) \theta\left(2 \xi_{a}+\beta \eta\right) \theta_{1}\left(2(\mathrm{~N}+2-\beta)-\sum_{b=1}^{8} \zeta_{-b} \mid 2 \omega\right) \theta_{1}\left(2 \zeta_{a}^{(0)} \mid 2 \omega\right) \theta_{2}^{2 \mathrm{~N}}\left(\zeta_{a}^{(0)} \mid 2 \omega\right)},
$$

$h_{n} \in\{0,1\}, n \in\{1, \ldots, \mathbf{N}\}$. It is important pointing out that the states $|\beta, \mathbf{h}\rangle$ are well defined states being their definition independent on the order of operator $\mathcal{D}_{-}\left(-\zeta_{b}^{(0)} \mid \beta\right)$ as one can verify directly by using the commutation relations (3.43) and the $\beta$-parity relation (3.45).

Theorem 4.3. Right $\mathcal{B}_{-}(\lambda \mid \beta)$ SOV-representations If (4.9) and

$$
\tilde{K}_{-}(\lambda \mid-\beta+2)_{21} \neq 0,
$$

are satisfied, then the states $|\beta, \boldsymbol{h}\rangle$ defines a basis formed out of $\mathcal{B}_{-}(\lambda \mid \beta)$-pseudo-eigenstates:

$$
\mathcal{B}_{-}(\lambda \mid \beta)|\beta, \boldsymbol{h}\rangle=|\beta+2, \boldsymbol{h}\rangle \overline{\mathrm{B}}_{\beta, \boldsymbol{h}}(\lambda),
$$

where:

$$
\overline{\mathrm{B}}_{\beta, \boldsymbol{h}}(\lambda) \equiv(-1)^{\mathrm{N}} \tilde{K}_{-}(\lambda \mid-\beta+2)_{21} \frac{\theta_{4}(2 \lambda-\eta \mid 2 \omega) \theta(\lambda+(\alpha+1 / 2) \eta) \theta(\eta(\beta-\mathrm{N}))}{\theta(\eta \beta)\left(\mathrm{N}_{\beta} / \mathrm{N}_{\beta+2}\right)\left(\prod_{n=1}^{\mathrm{N}} k_{n}^{(\beta)} / k_{n}^{(\beta+2)}\right)} a_{\boldsymbol{h}}(\lambda) a_{\boldsymbol{h}}(-\lambda) .
$$

Moreover, $\mathcal{D}_{-}(\lambda \mid \beta)$ is an order $4 \mathrm{~N}+8$ elliptic polynomials of periods $\pi$ and $2 \pi \omega$ :

$$
\begin{aligned}
\mathcal{D}_{-}(\lambda+2 \pi \omega \mid \beta) & =\left(-e^{-2 i \lambda} / q^{2}\right)^{4 \mathrm{~N}+8} e^{2 i \alpha_{\mathcal{D}_{-}(\beta)}} \mathcal{D}_{-}(\lambda \mid \beta), \\
\mathcal{D}_{-}(\lambda+\pi \mid \beta) & =\mathcal{D}_{-}(\lambda \mid \beta), \text { where } \alpha_{\mathcal{D}_{-}(\beta)} \equiv 2(\mathrm{~N}+2-\beta) \eta .
\end{aligned}
$$

Defined the operator $\mathcal{D}_{-}^{(0)}(\lambda \mid \beta)$ by the following action on the generic state $|\beta, \boldsymbol{h}\rangle$ :

$$
\begin{aligned}
\mathcal{D}_{-}^{(0)}(\lambda \mid \beta)|\beta, \boldsymbol{h}\rangle \equiv & \sum_{a=1}^{8} \frac{\theta_{1}\left(2(\mathrm{~N}+2-\beta)-\lambda-\sum_{b=1, b \neq a}^{8} \zeta_{-b} \mid 2 \omega\right)}{\theta_{1}\left(2(\mathrm{~N}+2-\beta)-\sum_{b=1}^{8} \zeta_{-b} \mid 2 \omega\right)} \frac{a_{\boldsymbol{h}}(\lambda) a_{\boldsymbol{h}}(-\lambda)}{a_{\boldsymbol{h}}\left(\zeta_{-a}\right) a_{\boldsymbol{h}}\left(-\zeta_{-a}\right)} \\
& \times \prod_{b=1, b \neq a}^{8} \frac{\theta_{1}\left(\lambda-\zeta_{-b} \mid 2 \omega\right)}{\theta_{1}\left(\zeta_{-a}-\zeta_{-b} \mid 2 \omega\right)} \mathcal{D}_{-}\left(\zeta_{-a} \mid \beta\right)|\beta, \boldsymbol{h}\rangle,
\end{aligned}
$$

then the operator:

$$
\widetilde{\mathcal{D}}_{-}(\lambda \mid \beta) \equiv \mathcal{D}_{-}(\lambda \mid \beta)-\mathcal{D}_{-}^{(0)}(\lambda \mid \beta),
$$

has the following action on the generic state $|\beta, \boldsymbol{h}\rangle$ :

$$
\begin{aligned}
\widetilde{\mathcal{D}}_{-}(\lambda \mid \beta)|\beta, \boldsymbol{h}\rangle & =\sum_{a=1}^{2 \mathrm{~N}} T_{a}^{-\varphi_{a}}|\beta, \boldsymbol{h}\rangle \mathrm{D}_{-}\left(\zeta_{a}^{\left(h_{a}\right)}\right) \frac{\theta_{1}\left(\lambda+\zeta_{a}^{\left(h_{a}\right)} \mid 2 \omega\right) \theta_{2}^{2 \mathrm{~N}}(\lambda \mid 2 \omega)}{\theta_{1}\left(2 \zeta_{a}^{\left(h_{a}\right)} \mid 2 \omega\right) \theta_{2}^{2 \mathrm{~N}}\left(\zeta_{a}^{\left(h_{a}\right)} \mid 2 \omega\right)} \prod_{\substack{b=1 \\
b \neq a}}^{\mathrm{N} m o d \mathrm{~N}} \frac{\frac{\theta_{4}^{2}(\lambda \mid 2 \omega)}{\theta_{2}^{2}(\lambda \mid 2 \omega)}-\frac{\theta_{4}^{2}\left(\zeta_{b}^{\left(h_{b}\right)} \mid 2 \omega\right)}{\theta_{2}^{2}\left(\zeta_{a}^{\left(h_{a}\right)} \mid 2 \omega\right)}-\frac{\theta_{b}^{2}\left(\zeta_{b}^{\left(h_{b}\right)} \mid 2 \omega\right)}{\left.\theta_{b}^{\left(h_{b}\right)} \mid 2 \omega\right)}}{\theta_{2}^{2}\left(\zeta_{b}^{\left(h_{b}\right)} \mid 2 \omega\right)} \\
& \times \frac{\theta_{4}(2 \lambda-\eta \mid 2 \omega) \theta_{1}(2 \lambda-\eta \mid 2 \omega) \theta_{1}\left(2(\mathrm{~N}+2-\beta)+\zeta_{a}^{\left(h_{a}\right)}-\lambda-\sum_{b=1}^{8} \zeta_{-b} \mid 2 \omega\right)}{\theta_{4}\left(2 \zeta_{a}^{\left(h_{a}\right)}-\eta \mid 2 \omega\right) \theta_{1}\left(2 \zeta_{a}^{\left(h_{a}\right)}-\eta \mid 2 \omega\right) \theta_{1}\left(2(\mathrm{~N}+2-\beta)-\sum_{b=1}^{8} \zeta_{-b} \mid 2 \omega\right)}
\end{aligned}
$$

\footnotetext{
${ }^{9}$ Note that this is the condition (4.11) in $\beta^{\prime}$ for $\beta^{\prime}=\beta-2$.
} 
where:

$$
\mathrm{D}_{-}\left(\zeta_{a}^{\left(h_{a}\right)}\right)=\left(k_{a}^{(\beta)}\right)^{\varphi_{a}} \mathrm{~A}_{-}\left(\zeta_{a}^{\left(h_{a}\right)}-2 \varphi_{a} \xi_{a}\right), \quad T_{a}^{ \pm}\left|\beta, h_{1}, \ldots, h_{a}, \ldots, h_{\mathrm{N}}\right\rangle=\left|\beta, h_{1}, \ldots, h_{a} \pm 1, \ldots, h_{\mathrm{N}}\right\rangle .
$$

Proof. The proof follows as in the previous theorem. Let us first prove that $\overline{|\beta\rangle}$ is a right $\mathcal{B}_{-}(\lambda \mid \beta)$-pseudo-eigenstate. From the Proposition 4.2 and the following boundary-bulk decomposition:

$$
\begin{aligned}
\frac{\mathcal{C}_{-}(\lambda \mid \beta)}{\theta_{4}(2 \lambda-\eta \mid 2 \omega) \theta(\lambda+(\alpha+1 / 2) \eta)} & =\tilde{K}_{-}(\lambda \mid \beta)_{11} C(\lambda \mid \beta-2) \bar{A}(\lambda \mid \beta-1)+\tilde{K}_{-}(\lambda \mid \beta)_{22} D(\lambda \mid \beta-2) \bar{C}(\lambda \mid \beta-1) \\
& +\tilde{K}_{-}(\lambda \mid \beta)_{12} C(\lambda \mid \beta-2) \bar{C}(\lambda \mid \beta-1)+\tilde{K}_{-}(\lambda \mid \beta)_{21} D(\lambda \mid \beta-2) \bar{A}(\lambda \mid \beta-1),
\end{aligned}
$$

it follows that the state $|\beta\rangle$ is a right $\mathcal{C}_{-}(\lambda \mid \beta)$-pseudo-eigenstate; i.e. it holds:

$$
\mathcal{C}_{-}(\lambda \mid \beta)|\beta\rangle=|\beta-2\rangle \mathbf{C}_{\beta}(\lambda)
$$

where:

$$
\mathrm{C}_{\beta}(\lambda)=(-1)^{\mathrm{N}} \tilde{K}_{-}(\lambda \mid \beta)_{21} \theta_{4}(2 \lambda-\eta \mid 2 \omega) \theta(\lambda+(\alpha+1 / 2) \eta) \frac{\theta(\eta(\mathrm{N}+\beta-2))}{\theta(\eta(\beta-2))} a_{\mathbf{1}}(\lambda) a_{\mathbf{1}}(-\lambda) .
$$

Then from the identity (3.45), it follows that the formula (4.76) is equivalent to the following one:

$$
\mathcal{B}_{-}(\lambda \mid \beta) \overline{|\beta\rangle}=\overline{|\beta+2\rangle} \mathrm{C}_{-\beta+2}(\lambda) .
$$

Then by using the identities (4.78) and the commutation relations (3.42) and the formulae:

$$
\mathcal{D}_{-}\left(-\xi_{n}-\eta / 2 \mid \beta\right) \overline{|\beta\rangle}=\underline{0}, \mathcal{D}_{-}\left(\xi_{n}+\eta / 2 \mid \beta\right) \overline{|\beta\rangle} \neq \underline{0},
$$

the states (4.63) are proven to be non-zero $\mathcal{B}_{-}(\lambda \mid \beta)$-pseudo-eigenstates with pseudo-eigenvalues $\overline{\mathrm{B}}_{\beta, \mathbf{h}}(\lambda)$ which then forms a basis of $\mathcal{R}_{\mathrm{N}}$. The fact that the operator $\mathcal{D}_{-}(\lambda \mid \beta)$ is an order $4 \mathrm{~N}+8$ elliptic polynomials of periods $\pi$ and $2 \pi \omega$ which satisfies (4.41)-(4.42) can be simply derived from (4.40) by using the commutation relations (3.42). Indeed, shifting the variable $\lambda_{2}$ in $\lambda_{2}+2 \pi \omega$ and using the transformation properties (4.40) and (4.54), we get:

$$
\begin{aligned}
f_{\mathcal{D}_{-}(\beta)}\left(\lambda_{2}\right) \mathcal{B}_{-}\left(\lambda_{1} \mid \beta\right) \mathcal{D}_{-}\left(\lambda_{2} \mid \beta\right) & =\frac{\theta\left(\lambda_{1}-\lambda_{2}+\eta\right) \theta\left(\lambda_{2}+\lambda_{1}-\eta\right)}{\theta\left(\lambda_{1}-\lambda_{2}\right) \theta\left(\lambda_{1}+\lambda_{2}\right)} e^{8 i \eta} f_{\mathcal{D}_{-}(\beta+2)}\left(\lambda_{2}\right) \mathcal{D}_{-}\left(\lambda_{2} \mid \beta+2\right) \mathcal{B}_{-}\left(\lambda_{1} \mid \beta\right) \\
& -\frac{\theta\left(\lambda_{2}-\lambda_{1}+(1+\beta) \eta\right) \theta\left(\lambda_{2}+\lambda_{1}-\eta\right)}{\theta\left(\lambda_{1}-\lambda_{2}\right) \theta\left(\lambda_{2}+\lambda_{1}\right) \theta((1+\beta) \eta)} e^{-4 i \beta \eta} f_{\mathcal{B}_{-}(\beta)}\left(\lambda_{2}\right) \mathcal{D}_{-}\left(\lambda_{1} \mid \beta+2\right) \mathcal{B}_{-}\left(\lambda_{2} \mid \beta\right) \\
& -\frac{\theta(\eta) \theta\left(\lambda_{2}+\lambda_{1}+\beta \eta\right)}{\theta\left(\lambda_{2}+\lambda_{1}\right) \theta((1+\beta) \eta)} e^{-4 i \beta \eta} f_{\mathcal{B}_{-}(\beta)}\left(\lambda_{2}\right) \mathcal{A}_{-}\left(\lambda_{1} \mid \beta+2\right) \mathcal{B}_{-}\left(\lambda_{2} \mid \beta\right)
\end{aligned}
$$

where we have defined:

$$
\mathcal{D}_{-}(\lambda+2 \pi \omega \mid \beta)=f_{\mathcal{D}_{-}(\beta)}(\lambda) \mathcal{D}_{-}(\lambda \mid \beta)
$$

which implies:

$$
f_{\mathcal{D}_{-}(\beta)}(\lambda) \equiv\left(-e^{-2 i \lambda} / q^{2}\right)^{4 \mathrm{~N}+8} e^{2 i \alpha_{\mathcal{D}_{-}(\beta)}} \text { where } \alpha_{\mathcal{D}_{-}(\beta)} \equiv 2(\mathrm{~N}+2-\beta) \eta
$$

Moreover, by the definition (4.71) it is simple to argue that the operators $\mathcal{D}_{-}^{(0)}(\lambda \mid \beta)$ is also an order $4 \mathrm{~N}+8$ elliptic polynomials of periods $\pi$ and $2 \pi \omega$ which satisfies (4.68) and (4.69) and then the same is true for $\widetilde{\mathcal{D}}_{-}(\lambda \mid \beta)$. This properties together with the identities:

$$
\widetilde{\mathcal{D}}_{-}\left(\zeta_{-a} \mid \beta\right) \equiv \underline{0} \text { for any } a \in\{1, \ldots, 8\},
$$

implies the interpolation formula (4.73). 


\subsection{SOV-decomposition of the identity}

We can derive some important information analyzing the change of basis from the spin basis:

$$
\langle\mathbf{h}| \equiv \otimes_{n=1}^{\mathrm{N}}\left\langle 2 h_{n}-1, n\right| \quad \text { and } \quad|\mathbf{h}\rangle \equiv \otimes_{n=1}^{\mathrm{N}}\left|2 h_{n}-1, n\right\rangle,
$$

to the SOV-basis. This change of basis can be characterized in terms of the $2^{\mathrm{N}} \times 2^{\mathrm{N}}$ matrices $U^{(L, \beta)}$ and $U^{(R, \beta)}$ :

$$
\langle\beta, \mathbf{h}|=\langle\mathbf{h}| U^{(L, \beta)}=\sum_{i=1}^{2^{N}} U_{\varkappa(\mathbf{h}), i}^{(L, \beta)}\left\langle\varkappa^{-1}(i)\right| \text { and }|\beta, \mathbf{h}\rangle=U^{(R, \beta)}|\mathbf{h}\rangle=\sum_{i=1}^{2^{N}} U_{i, \varkappa(\mathbf{h})}^{(R, \beta)}\left|\varkappa^{-1}(i)\right\rangle,
$$

where:

$$
\varkappa: \mathbf{h} \in\{0,1\}^{\mathrm{N}} \rightarrow \varkappa(\mathbf{h}) \equiv 1+\sum_{a=1}^{\mathrm{N}} 2^{(a-1)} h_{a} \in\left\{1, \ldots, 2^{\mathrm{N}}\right\},
$$

is an isomorphism between the sets $\{0,1\}^{\mathrm{N}}$ and $\left\{1, \ldots, 2^{\mathrm{N}}\right\}$. The pseudo-diagonalizability of $\mathcal{B}_{-}(\lambda \mid \beta)$ implies that the matrices $U^{(L, \epsilon)}$ and $U^{(R, \epsilon)}$ are invertible matrices satisfying the following identities:

$$
U^{(L, \beta)} \mathcal{B}_{-}(\lambda \mid \beta)=\Delta_{\mathcal{B}_{-}}^{L}(\lambda \mid \beta) U^{(L, \beta-2)}, \quad \mathcal{B}_{-}(\lambda \mid \beta) U^{(R, \beta)}=U^{(R, \beta+2)} \Delta_{\mathcal{B}_{-}}^{R}(\lambda \mid \beta) .
$$

Here $\Delta_{\mathcal{B}_{-}}^{L / R}(\lambda \mid \beta)$ is the $2^{\mathrm{N}} \times 2^{\mathrm{N}}$ diagonal matrix whose elements, for the simplicity of the $\mathcal{B}_{\epsilon}$-pseudo-spectrum, read:

$$
\left(\Delta_{\mathcal{B}_{-}}^{L}(\lambda \mid \beta)\right)_{i, j} \equiv \delta_{i, j} \mathrm{~B}_{\beta, \varkappa^{-1}(i)}(\lambda \mid \beta), \quad\left(\Delta_{\mathcal{B}_{-}}^{R}(\lambda \mid \beta)\right)_{i, j} \equiv \delta_{i, j} \overline{\mathrm{B}}_{\beta, \varkappa^{-1}(i)}(\lambda \mid \beta), \quad \forall i, j \in\left\{1, \ldots, 2^{\mathrm{N}}\right\} .
$$

Moreover, we can prove:

Proposition 4.3. Let us define the following $2^{\mathrm{N}} \times 2^{\mathrm{N}}$ matrix:

$$
M \equiv U^{(L, \beta-2)} U^{(R, \beta)}
$$

then it is diagonal and it explicitly reads:

$$
M_{\varkappa(\boldsymbol{h}) \varkappa(\boldsymbol{k})}=\langle\beta-2, \boldsymbol{h} \mid \beta, \boldsymbol{k}\rangle=\delta_{\varkappa(\boldsymbol{h}) \varkappa(\boldsymbol{k})} \prod_{1 \leq b<a \leq \mathrm{N}} \frac{1}{\eta_{a}^{\left(h_{a}\right)}-\eta_{b}^{\left(h_{b}\right)}},
$$

once the function $\mathrm{N}_{\beta}$ entering in the pseudo-eigenstates normalization is defined by:

$$
\mathrm{N}_{\beta}=\left[\prod_{1 \leq b<a \leq \mathrm{N}}\left(\eta_{a}^{(1)}-\eta_{a}^{(1)}\right)\langle\beta-2|\left(\prod_{n=1}^{\mathrm{N}} \mathcal{A}_{-}\left(\eta / 2-\xi_{n} \mid \beta\right) / \mathrm{A}_{-}\left(\eta / 2-\xi_{n}\right)\right) \overline{|\beta\rangle}\right]^{1 / 2},
$$

and

$$
\eta_{a}^{\left(h_{a}\right)} \equiv \frac{\theta_{4}^{2}\left(\left(\xi_{a}+\left(h_{a}-\frac{1}{2}\right) \eta \mid 2 \omega\right)\right.}{\theta_{2}^{2}\left(\left(\xi_{a}+\left(h_{a}-\frac{1}{2}\right) \eta \mid 2 \omega\right)\right.} .
$$

Proof. The occurence of $\delta_{\varkappa(\mathbf{h}) \varkappa(\mathbf{k})}$ in (4.90) follows by the following identities of matrix elements:

$$
\overline{\mathrm{B}}_{\beta, \mathbf{k}}(\lambda \mid \beta)\langle\beta, \mathbf{h} \mid \beta+2, \mathbf{k}\rangle=\left\langle\beta, \mathbf{h}\left|\mathcal{B}_{-}(\lambda \mid \beta)\right| \beta, \mathbf{k}\right\rangle=\mathrm{B}_{\beta, \mathbf{h}}(\lambda \mid \beta)\langle\beta-2, \mathbf{h} \mid \beta, \mathbf{k}\rangle,
$$

indeed the condition $\mathbf{h} \neq \mathbf{k}$ implies $\exists n \in\{1, \ldots, \mathbf{N}\}$ such that $h_{n} \neq k_{n}$ and then it implies:

$$
\overline{\mathrm{B}}_{\beta, \mathbf{k}}\left(\zeta_{n}^{\left(k_{n}\right)} \mid \beta\right)=0, \mathrm{~B}_{\beta, \mathbf{h}}\left(\zeta_{n}^{\left(k_{n}\right)} \mid \beta\right) \neq 0,
$$


and so:

$$
\langle\beta-2, \mathbf{h} \mid \beta, \mathbf{k}\rangle \propto \delta_{\varkappa(\mathbf{h}) \varkappa(\mathbf{k})} .
$$

The diagonal elements $M_{\varkappa(\mathbf{h}) \varkappa(\mathbf{h})}$ are obtained by computing

$$
\theta_{a}^{(\beta)} \equiv\left\langle\beta-2, h_{1}, \ldots, h_{a}=1, \ldots, h_{\mathrm{N}}\left|\widetilde{\mathcal{D}}_{-}\left(\xi_{a}+\eta / 2 \mid \beta\right)\right| \beta, h_{1}, \ldots, h_{a}=0, \ldots, h_{\mathrm{N}}\right\rangle
$$

for any $a \in\{1, \ldots, N\}$. Being:

$$
\left\langle\beta-2, h_{1}, \ldots, h_{a}=1, \ldots, h_{\mathrm{N}}\right| \tilde{\mathcal{D}}_{-}\left(\xi_{a}+\eta / 2 \mid \beta\right)=\left\langle\beta-2, h_{1}, \ldots, h_{a}=1, \ldots, h_{\mathrm{N}}\right| \mathcal{D}_{-}\left(\xi_{a}+\eta / 2 \mid \beta\right),
$$

then using the decomposition (3.26) and the fact that:

$$
\left\langle\beta-2, h_{1}, \ldots, h_{a}=1, \ldots, h_{\mathrm{N}}\right| \mathcal{A}_{-}\left(-\left(\xi_{a}+\eta / 2\right) \mid \beta\right)=\underline{0}
$$

it holds:

$$
\begin{aligned}
& \left\langle\beta-2, h_{1}, \ldots, h_{a}=1, \ldots, h_{\mathrm{N}}\right| \tilde{\mathcal{D}}_{-}\left(\xi_{a}+\eta / 2 \mid \beta\right) \\
& =\frac{\theta(\eta) \theta\left(2 \xi_{a}+\beta \eta\right)}{\theta\left(2 \xi_{a}+\eta\right) \theta(\beta \eta)}\left\langle\beta-2, h_{1}, \ldots, h_{a}=1, \ldots, h_{\mathrm{N}}\right| \mathcal{A}_{-}\left(\xi_{a}+\eta / 2 \mid \beta\right) \\
= & \frac{\theta(\eta) \theta\left(2 \xi_{a}+\beta \eta\right)}{\theta\left(2 \xi_{a}+\eta\right) \theta(\beta \eta)} \mathrm{A}_{-}\left(\eta / 2+\xi_{a}\right)\left\langle\beta-2, h_{1}, \ldots, h_{a}=0, \ldots, h_{\mathrm{N}}\right|,
\end{aligned}
$$

and then we get:

$$
\theta_{a}^{(\beta)}=\frac{\theta(\eta) \theta\left(2 \xi_{a}+\beta \eta\right)}{\theta\left(2 \xi_{a}+\eta\right) \theta(\beta \eta)} \mathrm{A}_{-}\left(\eta / 2+\xi_{a}\right)\left\langle\beta-2, h_{1}, \ldots, h_{a}=0, \ldots, h_{\mathrm{N}} \mid \beta, h_{1}, \ldots, h_{a}=0, \ldots, h_{\mathrm{N}}\right\rangle .
$$

On the other hand the right action of the operator $\widetilde{\mathcal{D}}_{-}\left(\xi_{a}+\eta / 2 \mid \beta\right)$ and the condition (4.95) implies:

$$
\begin{aligned}
& \theta_{a}^{(\beta)}=\left(k_{a}^{(\beta)}\right)^{-1} \mathrm{~A}_{-}\left(\eta / 2+\xi_{a}\right) \frac{\theta_{1}\left(2(\mathrm{~N}+2-\beta)-\sum_{b=1}^{8} \zeta_{-b}-2 \xi_{a} \mid 2 \omega\right) \theta_{1}(\eta \mid 2 \omega) \theta_{2}^{2 \mathrm{~N}}\left(\zeta_{a}^{(1)} \mid 2 \omega\right)}{\theta_{1}\left(2(\mathrm{~N}+2-\beta)-\sum_{b=1}^{8} \zeta_{-b} \mid 2 \omega\right) \theta_{1}\left(2 \zeta_{a}^{(0)} \mid 2 \omega\right) \theta_{2}^{2 \mathrm{~N}}\left(\zeta_{a}^{(0)} \mid 2 \omega\right)}
\end{aligned}
$$

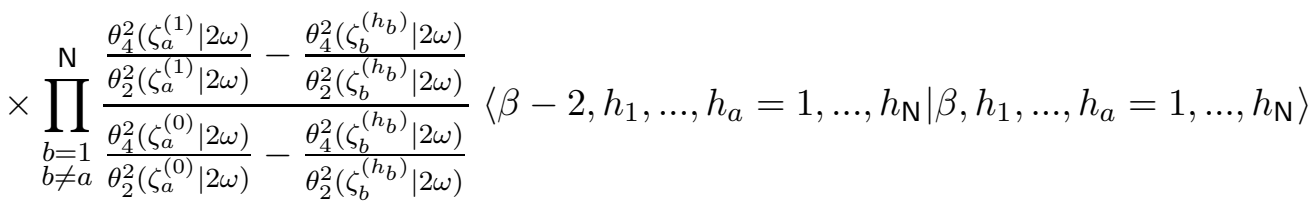

so that it holds:

$$
\frac{\left\langle\beta-2, h_{1}, \ldots, h_{a}=0, \ldots, h_{\mathrm{N}} \mid \beta, h_{1}, \ldots, h_{a}=0, \ldots, h_{\mathrm{N}}\right\rangle}{\left\langle\beta-2, h_{1}, \ldots, h_{a}=1, \ldots, h_{\mathrm{N}} \mid \beta, h_{1}, \ldots, h_{a}=1, \ldots, h_{\mathrm{N}}\right\rangle}=\prod_{\substack{b=1 \\ b \neq a}}^{\mathrm{N}} \frac{\frac{\theta_{4}^{2}\left(\zeta_{2}^{(1)} \mid 2 \omega\right)}{\theta_{2}^{2}\left(\zeta_{a}^{(1)} \mid 2 \omega\right)}-\frac{\theta_{4}^{2}\left(\zeta_{b}^{\left(h_{b}\right)} \mid 2 \omega\right)}{\theta_{2}^{2}\left(\zeta_{b}^{\left(h_{b}\right)} \mid 2 \omega\right)}}{\theta_{2}^{2}\left(\zeta_{a}^{(0)} \mid 2 \omega\right)}-\frac{\theta_{4}^{2}\left(\zeta_{b}^{\left(h_{b}\right)} \mid 2 \omega\right)}{\theta_{2}^{2}\left(\zeta_{b}^{\left(h_{b}\right)} \mid 2 \omega\right)},
$$

from which one can prove:

$$
\frac{\left\langle\beta-2, h_{1}, \ldots, h_{\mathrm{N}} \mid \beta, h_{1}, \ldots, h_{\mathrm{N}}\right\rangle}{\langle\beta-2,1, \ldots, 1 \mid \beta, 1, \ldots, 1\rangle}=\prod_{1 \leq b<a \leq \mathrm{N}} \frac{\eta_{a}^{(1)}-\eta_{b}^{(1)}}{\eta_{a}^{\left(h_{a}\right)}-\eta_{b}^{\left(h_{b}\right)}} .
$$

This last identity implies (4.90) being

$$
\langle\beta-2,1, \ldots, 1 \mid \beta, 1, \ldots, 1\rangle=\prod_{1 \leq b<a \leq \mathrm{N}} \frac{1}{\eta_{a}^{(1)}-\eta_{b}^{(1)}},
$$

by our definition of the normalization $\mathrm{N}_{\beta}$. 
The previous results allow to write the following spectral decomposition of the identity $\mathbb{I}$ :

$$
\mathbb{I} \equiv \sum_{i=1}^{2^{\mathrm{N}}} \mu\left|\beta, \varkappa^{-1}(i)\right\rangle\left\langle\beta-2, \varkappa^{-1}(i)\right|
$$

where $\mu \equiv\left(\left\langle\beta-2, \varkappa^{-1}(i) \mid \beta, \varkappa^{-1}(i)\right\rangle\right)^{-1}$ is the analogous (pseudo-measure) of the so-called Sklyanin's measure in the 8 -vertex reflection algebra representations, which reads explicitly:

$$
\mathbb{I} \equiv \sum_{h_{1}, \ldots, h_{\mathrm{N}}=0}^{1} \prod_{1 \leq b<a \leq \mathrm{N}}\left(\eta_{a}^{\left(h_{a}\right)}-\eta_{a}^{\left(h_{a}\right)}\right)\left|\beta, h_{1}, \ldots, h_{\mathrm{N}}\right\rangle\left\langle\beta-2, h_{1}, \ldots, h_{\mathrm{N}}\right| .
$$

\section{Separate variable characterization of transfer matrix spectrum}

In this section, we show how the SOV approach allows to write eigenvalues and eigenstates for the transfer matrix associated to the most general representation of the 8-vertex reflection algebra once the gauge transformations are used. The SOV characterization here presented is the natural generalization to the 8-vertex reflection algebra case of those first derived for the 6-vertex case in [1].

Theorem 5.1. Keeping completely arbitrary the six boundary parameters and using the freedom in the choice of the gauge parameters to impose (3.65), then:

$\left.I_{b}\right)$ the left representation for which the one parameter family $\mathcal{B}_{-}(\lambda \mid \beta)$ is pseudo-diagonal defines a left SOV representation for the spectral problem of the transfer matrix $\mathcal{T}(\lambda)$.

$\left.I I_{b}\right)$ the right representation for which the one parameter family $\mathcal{B}_{-}(\lambda \mid \beta+2)$ is pseudo-diagonal defines a right $S O V$ representation for the spectral problem of the transfer matrix $\mathcal{T}(\lambda)$.

Keeping completely arbitrary the six boundary parameters and using the freedom in the choice of the gauge parameters to impose:

$$
K_{+}^{(L)}(\lambda \mid \beta)_{21}=0
$$

then:

$\left.I_{c}\right)$ the left representation for which the one parameter family $\mathcal{C}_{-}(\lambda \mid \beta+4)$ is pseudo-diagonal defines a left SOV representation for the spectral problem of the transfer matrix $\mathcal{T}(\lambda)$.

$\left.I I_{c}\right)$ the right representation for which the one parameter family $\mathcal{C}_{-}(\lambda \mid \beta+2)$ is pseudo-diagonal defines a right SOV representation for the spectral problem of the transfer matrix $\mathcal{T}(\lambda)$.

Here, we will present these SOV constructions in this way proving the theorem only in the cases $\mathrm{I}_{b}$ ) and $\mathrm{II}_{b}$ ) as for the cases $\mathrm{I}_{c}$ ) and $\mathrm{II}_{c}$ ) these can be inferred mainly by using the $\beta$-symmetries defined in Lemma 3.2 .

Lemma 5.1. Let us denote with $\Sigma_{\mathcal{T}}$ the set of the eigenvalue functions of the transfer matrix $\mathcal{T}(\lambda)$, then any $\mathrm{t}(\lambda) \in \Sigma_{\mathcal{T}}$ is even in $\lambda$ and it satisfies the following quasi-periodicity properties in $\lambda$ w.r.t. the periods $\pi$ and $\pi \omega$ :

$$
\mathrm{t}(\lambda+\pi)=\mathrm{t}(\lambda), \mathrm{t}(\lambda+\pi \omega)=\left(e^{-2 i \lambda} / q\right)^{2 \mathrm{~N}+2} \mathrm{t}(\lambda) .
$$

Moreover, the following identities hold:

$$
\begin{aligned}
\mathrm{t}\left( \pm \zeta_{-1}\right) & =\frac{2 \theta_{2}(\eta \mid \omega) \theta_{4}^{2}\left(\zeta_{-} \mid 2 \omega\right) \theta_{4}^{2}\left(\zeta_{+} \mid 2 \omega\right)}{\theta_{2}(0 \mid \omega) \theta_{4}^{-1}(2 \eta \mid 2 \omega) \theta_{4}^{-1}(0 \mid 2 \omega)} \operatorname{det}_{q} M(0) \\
\mathrm{t}\left( \pm \zeta_{-2}\right) & =\frac{2 \theta_{2}(\eta \mid \omega) \prod_{\epsilon=+,-} \theta_{4}\left(\zeta_{\epsilon} \mid 2 \omega\right) \theta_{3}\left(\zeta_{\epsilon} \mid 2 \omega\right) \theta_{2}\left(\zeta_{\epsilon} \mid 2 \omega\right)}{\theta_{2}(0 \mid \omega) \theta_{1}\left(\zeta_{-} \mid 2 \omega\right) \theta_{1}\left(\zeta_{+} \mid 2 \omega\right) \theta_{4}^{-1}(2 \eta \mid 2 \omega) \theta_{4}^{-1}(0 \mid 2 \omega)} \operatorname{det}_{q} M(\pi / 2)
\end{aligned}
$$


while the following identities:

$$
\begin{gathered}
\lim _{\lambda \rightarrow \pm \zeta_{-3}} \theta_{4}(2 \lambda+\eta \mid 2 \omega) \theta_{4}(2 \lambda-\eta \mid 2 \omega) \mathrm{t}(\lambda)=4 \kappa_{-} \kappa_{+} \sinh \tau_{-} \sinh \tau_{+} e^{-2 i \sum_{a=1}^{\mathrm{N}} \zeta_{a}^{(0)}} \operatorname{det}_{q} M(-\pi \omega / 2) \\
\times \frac{\theta_{1}(\pi \omega \mid 2 \omega) \theta_{1}(2 \eta-\pi \omega \mid 2 \omega) \theta_{1}^{2}(\pi \omega / 2 \mid 2 \omega) \theta_{4}^{3}\left(\zeta_{-} \mid 2 \omega\right) \theta_{4}^{3}\left(\zeta_{+} \mid 2 \omega\right) \theta_{4}^{-4}(0 \mid 2 \omega)}{\theta_{1}\left(\zeta_{-} \mid 2 \omega\right) \theta_{1}\left(\zeta_{+} \mid 2 \omega\right)\left[\theta_{4}^{2}(\eta-\pi \omega / 2 \mid 2 \omega)+\theta_{1}^{2}(\eta-\pi \omega / 2 \mid 2 \omega)\right]^{-1}}, \\
\lim _{\lambda \rightarrow \pm \zeta_{-4}} \theta_{4}(2 \lambda+\eta \mid 2 \omega) \theta_{4}(2 \lambda-\eta \mid 2 \omega) \mathrm{t}(\lambda)=4 \kappa_{-} \kappa_{+} \cosh \tau_{-} \cosh \tau_{+} e^{-2 i \sum_{a=1}^{N} \zeta_{a}^{(0)}} \operatorname{det}_{q} M(-\pi(\omega+1) / 2) \\
\times \frac{\theta_{1}(\pi \omega \mid 2 \omega) \theta_{1}(2 \eta-\pi \omega \mid 2 \omega) \theta_{1}^{2}(\pi(\omega+1) / 2 \mid 2 \omega) \theta_{4}^{3}\left(\zeta_{-} \mid 2 \omega\right) \theta_{4}^{3}\left(\zeta_{+} \mid 2 \omega\right) \theta_{4}^{-4}(0 \mid 2 \omega)}{\theta_{1}\left(\zeta_{-} \mid 2 \omega\right) \theta_{1}\left(\zeta_{+} \mid 2 \omega\right)\left[\theta_{4}^{2}(\eta-\pi(\omega+1) / 2 \mid 2 \omega)-\theta_{1}^{2}(\eta-\pi(\omega+1) / 2 \mid 2 \omega)\right]^{-1}}
\end{gathered}
$$

fix the residues of $\mathrm{t}(\lambda)$ in the poles $\pm \zeta_{-3}$ and $\pm \zeta_{-4}$.

Proof. The transfer matrix $\mathcal{T}(\lambda)$ is an even function of $\lambda$ so the same is true for the $\mathrm{t}(\lambda) \in \Sigma_{\mathcal{T}}$. Moreover, it is simple to verify the following identities:

$$
\mathcal{U}_{-}(\eta / 2)=\theta_{4}^{4}\left(\zeta_{\epsilon} \mid 2 \omega\right) \operatorname{det}_{q} M(0) I_{0}, \quad \mathcal{U}_{-}(\eta / 2+\pi / 2)=\frac{\theta_{3}\left(\zeta_{\epsilon} \mid 2 \omega\right) \theta_{2}\left(\zeta_{\epsilon} \mid 2 \omega\right)}{\theta_{1}\left(\zeta_{\epsilon} \mid 2 \omega\right) \theta_{4}^{-1}\left(\zeta_{\epsilon} \mid 2 \omega\right)} \operatorname{det}_{q} M(\pi / 2) \sigma_{0}^{z},
$$

from which the following identities are derived:

$$
\begin{aligned}
& \mathcal{T}\left( \pm \zeta_{-1}^{(0)}\right)=\frac{2 \theta_{2}(\eta \mid \omega) \theta_{4}^{2}\left(\zeta_{-} \mid 2 \omega\right) \theta_{4}^{2}\left(\zeta_{+} \mid 2 \omega\right)}{\theta_{2}(0 \mid \omega) \theta_{4}^{-1}(2 \eta \mid 2 \omega) \theta_{4}^{-1}(0 \mid 2 \omega)} \operatorname{det}_{q} M(0), \\
& \mathcal{T}\left( \pm \zeta_{-2}^{(0)}\right)=\frac{2 \theta_{2}(\eta \mid \omega) \prod_{\epsilon=+,-} \theta_{4}\left(\zeta_{\epsilon} \mid 2 \omega\right) \theta_{3}\left(\zeta_{\epsilon} \mid 2 \omega\right) \theta_{2}\left(\zeta_{\epsilon} \mid 2 \omega\right)}{\theta_{2}(0 \mid \omega) \theta_{1}\left(\zeta_{-} \mid 2 \omega\right) \theta_{1}\left(\zeta_{+} \mid 2 \omega\right) \theta_{4}^{-1}(2 \eta \mid 2 \omega) \theta_{4}^{-1}(0 \mid 2 \omega)} \operatorname{det}_{q} M(\pi / 2),
\end{aligned}
$$

in this way proving (5.3) and (5.4). The boundary matrix $K_{\epsilon}\left(\lambda ; \zeta_{\epsilon}, \kappa_{\epsilon}, \tau_{\epsilon}\right)$ contains the function $\theta_{4}(2 \lambda+\epsilon \eta \mid 2 \omega)$, with $\epsilon=+$ or - , at the denominator of the off-diagonal elements, so it is simple to argue that for general values of the boundary parameters the transfer matrix $\mathcal{T}(\lambda)$ my have poles in the zeros of the functions $\theta_{4}(2 \lambda-\eta \mid 2 \omega) \theta_{4}(2 \lambda+$ $\eta \mid 2 \omega)$. The residues associated to these poles follows from the following identities:

$$
\begin{aligned}
\lim _{\lambda \rightarrow \pm \zeta_{-a}} \theta_{4}(2 \lambda-\eta \mid 2 \omega) \mathcal{U}_{-}(\lambda) & =-\kappa_{-} \frac{\theta_{1}(\pi \omega+(a-3) \pi \mid 2 \omega) \theta_{1}^{2}(\pi(\omega+a-3) / 2 \mid 2 \omega)}{\theta_{1}\left(\zeta_{-} \mid 2 \omega\right) \theta_{4}^{-3}\left(\zeta_{-} \mid 2 \omega\right) \theta_{4}^{2}(0 \mid 2 \omega)} e^{-2 i \sum_{n=1}^{N} \zeta_{n}^{(0)}} \\
& \times\left(e^{\tau_{-}}+(2 a-7) e^{-\tau_{-}}\right) \operatorname{det}_{q} M(-\pi(\omega+a-3) / 2)\left(\begin{array}{cc}
0 & 2 a-7 \\
1 & 0
\end{array}\right),
\end{aligned}
$$

for $a=3$ and 4 , which are derived by using the following identities:

$$
\begin{aligned}
\lim _{\lambda \rightarrow \pm \zeta_{-a}} \theta_{4}(2 \lambda-\eta \mid 2 \omega) K_{-}(\lambda) & =-\kappa_{-} \frac{\theta_{1}(\pi \omega+(a-3) \pi \mid 2 \omega) \theta_{1}^{2}(\pi(\omega+a-3) / 2 \mid 2 \omega)}{\theta_{1}\left(\zeta_{-} \mid 2 \omega\right) \theta_{4}^{-3}\left(\zeta_{-} \mid 2 \omega\right) \theta_{4}^{2}(0 \mid 2 \omega)} \\
& \times\left(e^{\tau_{-}}+(2 a-7) e^{-\tau_{-}}\right)\left(\begin{array}{cc}
0 & 2 a-7 \\
1 & 0
\end{array}\right),
\end{aligned}
$$

and

$$
M\left(\zeta_{-a}\right)=(-1)^{\mathrm{N}} e^{-2 i \sum_{n=1}^{\mathrm{N}} \zeta_{n}^{(0)}}\left(\begin{array}{cc}
0 & 2 a-7 \\
1 & 0
\end{array}\right) M\left(\eta-\zeta_{-a}\right)\left(\begin{array}{cc}
0 & 1 \\
2 a-7 & 0
\end{array}\right),
$$

for $a=3$ and 4 where this last identity follows from:

$$
\begin{aligned}
& \mathrm{a}\left(-\frac{\pi}{2}\left(\omega+\frac{(1-\epsilon)}{2}\right)-\xi_{n}\right)=-e^{-2 i \zeta_{n}^{(0)}} \mathrm{b}\left(\frac{\pi}{2}\left(\omega+\frac{(1-\epsilon)}{2}\right)-\xi_{n}\right), \\
& \mathrm{c}\left(-\frac{\pi}{2}\left(\omega+\frac{(1-\epsilon)}{2}\right)-\xi_{n}\right)=\epsilon e^{-2 i \zeta_{n}^{(0)}} \mathrm{d}\left(\frac{\pi}{2}\left(\omega+\frac{(1-\epsilon)}{2}\right)-\xi_{n}\right) .
\end{aligned}
$$


Let us associate to any $\mathrm{t}(\lambda) \in \Sigma_{\mathcal{T}}$ the following even functions in $\lambda$ :

$$
\widehat{\mathrm{t}}(\lambda) \equiv \theta_{4}(2 \lambda+\eta \mid 2 \omega) \theta_{4}(2 \lambda-\eta \mid 2 \omega) \mathrm{t}(\lambda),
$$

then for the previous lemma $\widehat{t}(\lambda)$ is an elliptic polynomials in $\lambda$ of order $2 N+6$ which satisfy the following quasiperiodicity properties in $\lambda$ w.r.t. the periods $\pi$ and $\pi \omega$ :

$$
\widehat{\mathfrak{t}}(\lambda+\pi)=\widehat{\mathrm{t}}(\lambda), \widehat{\mathrm{t}}(\lambda+\pi \omega)=\left(e^{-2 i \lambda} / q\right)^{2 \mathrm{~N}+6} \widehat{\mathrm{t}}(\lambda) .
$$

Moreover, $\widehat{\mathrm{t}}(\lambda)$ has values in the points $\pm \zeta_{-a}$ for $a=1,2,3$ and 4 which are independent from the particular choice of $\mathrm{t}(\lambda) \in \Sigma_{\mathcal{T}}$ and completely fixed by the previous lemma. Then defined:

$$
j(\lambda) \equiv \sum_{a=1}^{4} l_{-a}(\lambda) \widehat{\mathfrak{t}}\left(\zeta_{-a}\right),
$$

where:

$$
l_{a}(\lambda) \equiv \prod_{\substack{b=1 \\ b \neq a}}^{4} \frac{\theta\left(\lambda-\zeta_{-b}\right) \theta\left(\lambda+\zeta_{-b}\right)}{\theta\left(\zeta_{-a}-\zeta_{-b}\right) \theta\left(\zeta_{-a}+\zeta_{-b}\right)} \prod_{\substack{b=1 \\ b \neq a}}^{\mathrm{N}} \frac{\theta\left(\lambda-\zeta_{b}^{(0)}\right) \theta\left(\lambda+\zeta_{b}^{(0)}\right)}{\theta\left(\zeta_{a}^{(0)}-\zeta_{b}^{(0)}\right) \theta\left(\zeta_{a}^{(0)}+\zeta_{b}^{(0)}\right)} \forall a \in\{-4, \ldots, \mathrm{N}\},
$$

one can observe that the elliptic polynomial $j(\lambda)$ is independent from the particular choice of $\mathrm{t}(\lambda) \in \Sigma_{\mathcal{T}}$. We can now prove the following complete characterization of the transfer matrix spectrum:

Theorem 5.2. $\mathcal{T}(\lambda)$ has simple spectrum if (4.9) is satisfied and $\Sigma_{\mathcal{T}}$ admits the following characterization:

$$
\Sigma_{\mathcal{T}} \equiv\left\{\mathrm{t}(\lambda): \mathrm{t}(\lambda)=\frac{j(\lambda)+\sum_{a=1}^{\mathrm{N}} l_{a}(\lambda) x_{a}}{\theta_{4}(2 \lambda+\eta \mid 2 \omega) \theta_{4}(2 \lambda-\eta \mid 2 \omega)}, \quad \forall\left\{x_{1}, \ldots, x_{\mathrm{N}}\right\} \in \Sigma_{T}\right\},
$$

where $\Sigma_{T}$ is the set of the solutions to the following inhomogeneous system of $\mathrm{N}$ quadratic equations:

$$
x_{n} \sum_{a=1}^{\mathrm{N}} l_{a}\left(\zeta_{n}^{(1)}\right) x_{a}+x_{n} j\left(\zeta_{n}^{(1)}\right)=q_{n}, \quad q_{n} \equiv \widehat{\mathrm{A}}\left(\zeta_{n}^{(1)}\right) \widehat{\mathrm{A}}\left(-\zeta_{n}^{(0)}\right), \quad \forall n \in\{1, \ldots, \mathrm{N}\},
$$

in the $\mathrm{N}$ unknown $\left\{x_{1}, \ldots, x_{\mathrm{N}}\right\}$, where $\widehat{\mathrm{A}}(\lambda)$ is defined by:

$$
\widehat{\mathrm{A}}(\lambda) \equiv \theta_{4}(2 \lambda+\eta \mid 2 \omega) \theta_{4}(2 \lambda-\eta \mid 2 \omega) \mathrm{A}(\lambda), \quad \mathrm{A}(\lambda) \equiv \mathrm{a}_{+}(\lambda) \mathrm{A}_{-}(\lambda),
$$

where $\mathrm{A}(\lambda)$ satisfies the quantum determinant condition:

$$
\frac{\operatorname{det}_{q} K_{+}(\lambda) \operatorname{det}_{q} \mathcal{U}_{-}(\lambda)}{\theta(\eta+2 \lambda) \theta(\eta-2 \lambda)}=\mathrm{A}(\eta / 2-\lambda) \mathrm{A}(\lambda+\eta / 2) .
$$

R) If (4.11) is verified, the vector:

$$
|\mathrm{t}\rangle=\sum_{h_{1}, \ldots, h_{\mathrm{N}}=0}^{1} \prod_{a=1}^{\mathrm{N}} Q_{\mathrm{t}}\left(\zeta_{a}^{\left(h_{a}\right)}\right) \prod_{1 \leq b<a \leq \mathrm{N}}\left(\eta_{a}^{\left(h_{a}\right)}-\eta_{b}^{\left(h_{b}\right)}\right)\left|\beta+2, h_{1}, \ldots, h_{\mathrm{N}}\right\rangle,
$$

with coefficients:

$$
Q_{\mathrm{t}}\left(\zeta_{a}^{(1)}\right) / Q_{\mathrm{t}}\left(\zeta_{a}^{(0)}\right)=\mathrm{t}\left(\zeta_{a}^{(0)}\right) / \mathrm{A}\left(-\zeta_{a}^{(0)}\right),
$$

is the right $\mathcal{T}$-eigenstate corresponding to $\mathrm{t}(\lambda) \in \Sigma_{\mathcal{T}}$ uniquely defined up to an overall normalization. 
L) If (4.10) is verified, the covector

$$
\langle\mathrm{t}|=\sum_{h_{1}, \ldots, h_{\mathrm{N}}=0}^{1} \prod_{a=1}^{\mathrm{N}} \bar{Q}_{\mathrm{t}}\left(\zeta_{a}^{\left(h_{a}\right)}\right) \prod_{1 \leq b<a \leq \mathrm{N}}\left(\eta_{a}^{\left(h_{a}\right)}-\eta_{b}^{\left(h_{b}\right)}\right)\left\langle\beta, h_{1}, \ldots, h_{\mathrm{N}}\right|,
$$

with coefficients:

$$
\bar{Q}_{\mathrm{t}}\left(\zeta_{a}^{(1)}\right) / \bar{Q}_{\mathrm{t}}\left(\zeta_{a}^{(0)}\right)=\mathrm{t}\left(\zeta_{a}^{(0)}\right) /\left(\mathrm{d}_{+}\left(\zeta_{a}^{(1)}\right) \mathrm{D}_{-}\left(\zeta_{a}^{(1)}\right)\right)
$$

is the left $\mathcal{T}$-eigenstate corresponding to $\mathrm{t}(\lambda) \in \Sigma_{\mathcal{T}}$ uniquely defined up to an overall normalization.

Proof. The separate variables characterization of the spectral problem for $\mathcal{T}(\lambda)$ is reduced to the discrete system of $2^{\mathrm{N}}$ Baxter-like equations:

$$
\mathrm{t}\left(\zeta_{n}^{\left(h_{n}\right)}\right) \Psi_{\mathrm{t}}(\mathbf{h})=\mathrm{A}\left(\zeta_{n}^{\left(h_{n}\right)}\right) \Psi_{\mathrm{t}}\left(\mathbf{T}_{n}^{-}(\mathbf{h})\right)+\mathrm{A}\left(-\zeta_{n}^{\left(h_{n}\right)}\right) \Psi_{\mathrm{t}}\left(\mathrm{T}_{n}^{+}(\mathbf{h})\right)
$$

for any $n \in\{1, \ldots, N\}$ and $\mathbf{h} \in\{0,1\}^{\mathrm{N}}$. Here, the (wave-functions) $\Psi_{\mathbf{t}}(\mathbf{h})$ are the coefficient of the $\mathcal{T}$-eigenstate $|\mathrm{t}\rangle$ corresponding to the $\mathrm{t}(\lambda) \in \Sigma_{\mathcal{T}}$ in the right $\mathcal{B}_{-}$-SOV representation and the following notations are introduced:

$$
\mathbf{T}_{n}^{ \pm}(\mathbf{h}) \equiv\left(h_{1}, \ldots, h_{n} \pm 1, \ldots, h_{\mathrm{N}}\right) .
$$

This system of separate equations is derived from the identities:

$$
\mathrm{A}_{-}\left(\zeta_{n}^{(0)}\right)=\mathrm{A}_{-}\left(-\zeta_{n}^{(1)}\right)=0,
$$

once we compute the matrix elements:

$$
\left\langle\beta, h_{1}, \ldots, h_{n}, \ldots, h_{\mathrm{N}}\left|\mathcal{T}\left( \pm \zeta_{n}^{\left(h_{n}\right)}\right)\right| \mathrm{t}\right\rangle .
$$

Indeed (3.52) implies:

$$
\begin{aligned}
\mathrm{t}\left( \pm \zeta_{n}^{(0)}\right) \Psi_{\mathrm{t}}\left(h_{1}, \ldots, h_{n}\right. & \left.=0, \ldots, h_{\mathrm{N}}\right)= \\
& =\left\langle\beta, h_{1}, \ldots, h_{n}=0, \ldots, h_{\mathrm{N}}\left|\mathcal{T}\left(-\zeta_{n}^{(0)}\right)\right| \mathrm{t}\right\rangle \\
& =\mathrm{a}_{+}\left(-\zeta_{n}^{(0)}\right)\left\langle\beta, h_{1}, \ldots, h_{n}=0, \ldots, h_{\mathrm{N}}\left|\mathcal{A}_{-}\left(-\zeta_{n}^{(0)}\right)\right| \mathrm{t}\right\rangle \\
& =\mathrm{A}\left(-\zeta_{n}^{(0)}\right) \Psi_{\mathrm{t}}\left(h_{1}, \ldots, h_{n}=1, \ldots, h_{\mathrm{N}}\right) \\
& =\mathrm{A}\left(-\zeta_{n}^{(0)}\right) \Psi_{\mathrm{t}}\left(h_{1}, \ldots, h_{n}=1, \ldots, h_{\mathrm{N}}\right)+\mathrm{A}\left(\zeta_{n}^{(0)}\right) \Psi_{\mathrm{t}}\left(h_{1}, \ldots, h_{n}=-1, \ldots, h_{\mathrm{N}}\right),
\end{aligned}
$$

and

$$
\begin{aligned}
\mathrm{t}\left( \pm \zeta_{n}^{(1)}\right) \Psi_{\mathrm{t}}\left(h_{1}, \ldots, h_{n}\right. & \left.=1, \ldots, h_{\mathrm{N}}\right)= \\
& =\left\langle\beta, h_{1}, \ldots, h_{n}=1, \ldots, h_{\mathrm{N}}\left|\mathcal{T}\left(\zeta_{n}^{(1)}\right)\right| \mathrm{t}\right\rangle \\
& =\mathrm{a}_{+}\left(\zeta_{n}^{(1)}\right)\left\langle\beta, h_{1}, \ldots, h_{n}=1, \ldots, h_{\mathrm{N}}\left|\mathcal{A}_{-}\left(\zeta_{n}^{(1)}\right)\right| \mathrm{t}\right\rangle \\
& =\mathrm{A}\left(\zeta_{n}^{(1)}\right) \Psi_{\mathrm{t}}\left(h_{1}, \ldots, h_{n}=0, \ldots, h_{\mathrm{N}}\right) \\
& =\mathrm{A}\left(\zeta_{n}^{(1)}\right) \Psi_{\mathrm{t}}\left(h_{1}, \ldots, h_{n}=0, \ldots, h_{\mathrm{N}}\right)+\mathrm{A}\left(-\zeta_{n}^{(1)}\right) \Psi_{\mathrm{t}}\left(h_{1}, \ldots, h_{n}=2, \ldots, h_{\mathrm{N}}\right) .
\end{aligned}
$$

The system (5.29) is clearly equivalent to the system of homogeneous equations:

$$
\left(\begin{array}{cc}
\mathrm{t}\left( \pm \zeta_{n}^{(0)}\right) & -\mathrm{A}\left(-\zeta_{n}^{(0)}\right) \\
-\mathrm{A}\left(\zeta_{n}^{(1)}\right) & \mathrm{t}\left( \pm \zeta_{n}^{(1)}\right)
\end{array}\right)\left(\begin{array}{c}
\Psi_{\mathrm{t}}\left(h_{1}, \ldots, h_{n}=0, \ldots, h_{1}\right) \\
\Psi_{\mathrm{t}}\left(h_{1}, \ldots, h_{n}=1, \ldots, h_{1}\right)
\end{array}\right)=\left(\begin{array}{l}
0 \\
0
\end{array}\right)
$$


for any $n \in\{1, \ldots, \mathrm{N}\}$ with $h_{r \neq n} \in\{0,1\}$. Then the determinants of the $2 \times 2$ matrices in (5.35) must be zero for any $n \in\{1, \ldots, \mathrm{N}\}$ if $\mathrm{t}(\lambda) \in \Sigma_{\mathcal{T}}$, i.e. it holds:

$$
\mathrm{t}\left( \pm \zeta_{a}^{(0)}\right) \mathrm{t}\left( \pm \zeta_{a}^{(1)}\right)=\mathrm{A}\left(\zeta_{a}^{(1)}\right) \mathrm{A}\left(-\zeta_{a}^{(0)}\right), \quad \forall a \in\{1, \ldots, \mathrm{N}\} .
$$

Being

$$
\mathrm{A}\left(-\zeta_{n}^{(0)}\right) \neq 0 \text { and } \mathrm{A}\left(\zeta_{n}^{(1)}\right) \neq 0,
$$

then the matrices in (5.35) have all rank 1 and up to an overall normalization the solution is unique:

$$
\frac{\Psi_{\mathrm{t}}\left(h_{1}, \ldots, h_{n}=1, \ldots, h_{\mathrm{N}}\right)}{\Psi_{\mathrm{t}}\left(h_{1}, \ldots, h_{n}=0, \ldots, h_{\mathrm{N}}\right)}=\frac{\mathrm{t}\left(\zeta_{a}^{(0)}\right)}{\mathrm{A}\left(-\zeta_{a}^{(0)}\right)},
$$

for any $n \in\{1, \ldots, \mathrm{N}\}$ with $h_{r \neq n} \in\{0,1\}$. So for any fixed $\mathrm{t}(\lambda) \in \Sigma_{\mathcal{T}}$ the associate eigenspace is one dimensional $(\mathcal{T}(\lambda)$ has simple spectrum) and $|t\rangle$ defined by $(\underline{5.25})-(\underline{5.26})$ is the only corresponding eigenstate up to normalization. It is simple now to prove that the set $\Sigma_{\mathcal{T}}$ is included in the set of functions characterized by (5.21) and (5.22); indeed for any $\mathrm{t}(\lambda) \in \Sigma_{\mathcal{T}}$ the associated elliptic polynomial defined in (5.17) admits the following interpolation formula:

$$
\widehat{\mathrm{t}}(\lambda)=j(\lambda)+\sum_{a=1}^{\mathrm{N}} l_{a}(\lambda) \widehat{\mathrm{t}}\left(\zeta_{a}^{(0)}\right)
$$

as the functions $j(\lambda)$ and $l_{a}(\lambda)$, as well as $\widehat{\mathrm{t}}(\lambda)$, are even elliptic polynomials in $\lambda$ of order $2 \mathrm{~N}+6$ which satisfy the same quasi-periodicity properties (5.18) and the interpolation formula is given on the $2(\mathrm{~N}+4)$ points:

$$
\pm \zeta_{-4}, \ldots, \pm \zeta_{-1}, \pm \zeta_{1}^{(0)}, \ldots, \pm \zeta_{N}^{(0)}
$$

Then using (5.39) the system of equation (5.36) is equivalent to (5.22).

Let prove now the reverse inclusion of set of functions, i.e. let us prove that if $t(\lambda)$ is in the set of functions characterized by (5.21) and (5.22) then it is an element of $\Sigma_{\mathcal{T}}$. Indeed, taking the state $|t\rangle$ defined by (5.25) $-(5.26)$ the following identities are satisfied:

$$
\left\langle\beta, h_{1}, \ldots, h_{\mathrm{N}}\left|\mathcal{T}\left( \pm \zeta_{n}^{\left(h_{n}\right)}\right)\right| \mathrm{t}\right\rangle=\mathrm{t}\left( \pm \zeta_{n}^{\left(h_{n}\right)}\right)\left\langle\beta, h_{1}, \ldots, h_{\mathrm{N}} \mid \mathrm{t}\right\rangle \forall n \in\{1, \ldots, \mathrm{N}\}
$$

and

$$
\lim _{\lambda \rightarrow \pm \zeta_{-a}} \theta_{4}(2 \lambda+\eta \mid 2 \omega) \theta_{4}(2 \lambda-\eta \mid 2 \omega)\left\langle\beta, h_{1}, \ldots, h_{\mathrm{N}}|\mathcal{T}(\lambda)| \mathrm{t}\right\rangle=\widehat{\mathfrak{t}}\left( \pm \zeta_{-a}\right)\left\langle\beta, h_{1}, \ldots, h_{\mathrm{N}} \mid \mathrm{t}\right\rangle,
$$

for any $a=1,2,3,4$ and this implies:

$$
\left\langle\beta, h_{1}, \ldots, h_{\mathrm{N}}|\mathcal{T}(\lambda)| \mathrm{t}\right\rangle=\mathrm{t}(\lambda)\left\langle\beta, h_{1}, \ldots, h_{\mathrm{N}} \mid \mathrm{t}\right\rangle \quad \forall \lambda \in \mathbb{C},
$$

for any $\mathcal{B}_{-}(\mid \beta)$-pseudo-eigenstate $\left\langle\beta, h_{1}, \ldots, h_{\mathbf{N}}\right|$, i.e. $\mathbf{t}(\lambda) \in \Sigma_{\mathcal{T}}$ and $|\mathrm{t}\rangle$ is the corresponding $\mathcal{T}$-eigenstate. Finally, let us point out that the quantum determinant condition (5.24) follows from the definition (4.46) and the quantum determinant conditions (2.35) and (3.66), where this last identity holds when (3.65) is satisfied as proven in Lemma 3.3 Concerning the left $\mathcal{T}$-eigenstates the proof is done as above. Here one has to compute the matrix elements:

$$
\left\langle\mathrm{t}\left|\mathcal{T}\left(\zeta_{n}^{\left(h_{n}\right)}\right)\right| \beta+2, h_{1}, \ldots, h_{\mathrm{N}}\right\rangle,
$$

which by using the right $\mathcal{B}(\mid \beta)$-representation read:

$$
\mathrm{t}\left(\zeta_{n}^{\left(h_{n}\right)}\right) \bar{\Psi}_{\mathrm{t}}(\mathbf{h})=\mathrm{D}\left(\zeta_{n}^{\left(h_{n}\right)}\right) \bar{\Psi}_{\mathrm{t}}\left(\mathrm{T}_{n}^{-}(\mathbf{h})\right)+\mathrm{D}\left(-\zeta_{n}^{\left(h_{n}\right)}\right) \bar{\Psi}_{\mathrm{t}}\left(\mathrm{T}_{n}^{+}(\mathbf{h})\right), \quad \forall n \in\{1, \ldots, \mathrm{N}\}
$$

where:

$$
\bar{\Psi}_{\mathrm{t}}(\mathbf{h}) \equiv\left\langle\mathrm{t} \mid \beta+2, h_{1}, \ldots, h_{\mathrm{N}}\right\rangle, \mathrm{D}\left( \pm \zeta_{a}^{\left(h_{a}\right)}\right) \equiv \mathrm{d}_{+}\left( \pm \zeta_{a}^{\left(h_{a}\right)}\right) \mathrm{D}_{-}\left( \pm \zeta_{a}^{\left(h_{a}\right)}\right)
$$


Under the most general boundary conditions the above inhomogeneous system of quadratic equations provides the characterization of the spectrum and replaces the Bethe ansatz formulation which applies only when the parameters satisfy the linear relation derived in [64]. It is however interesting to get a reformulation of this characterization by functional equations and the construction of a Baxter Q-operator can be important in this direction. In a next paper we will provide this construction based only on the SOV characterization following the approach defined first in [49] and generalized in [50] for cyclic 6-vertex representations. In the roots of unit case and for the most general boundary conditions this construction will be proven to lead to a Baxter Q-operator which is an elliptic polynomial in spectral parameter $\lambda$ and so to a proof of completeness of the spectrum (eigenvalues and eigenstates) characterization in terms of a system of Bethe ansatz equations. Finally, we want to report that after the completion of this manuscript, we have remarked the interesting paper [83] which follows the series of recent papers [84] on integrable quantum models associated to spin-1/2 representations of both Yang-Baxter and reflection algebras. For these integrable quantum models T-Q functional equations have been introduced for the characterization of the transfer matrix eigenvalues by an ansatz using as starting point the identities relating the products of the transfer matrix eigenvalues and the quantum determinant in special points related to the inhomogeneities of the models. These identities can be proven directly at the operator level for example by using the annihilation identities of the generators of both the Yang-Baxter and reflection algebras for both the 6-vertex and 8-vertex cases. This approach was described for example in [56] in the case of the periodic transfer matrices associated to spin-1/2 representation of the 8-vertex Yang-Baxter algebra and in the case of the antiperiodic transfer matrix associated to the spin-1/2 representation of the dynamical 6-vertex Yang-Baxter algebra. In [83] these identities are derived using the reduction in zero to the permutation operator of both the 8-vertex and 6-vertex R-matrix. The link with the separation of variables approach is very simple to explain in all the integrable quantum models analyzed so far and associated to representations defined on spin-1/2 quantum chains [1, 2, 54, 56, 59] the compatibility conditions of the transfer matrix separate equations, i.e. the system of Baxter like equations of type (5.29), are just the mentioned identities involving product of transfer matrices and quantum determinant (5.36). In the SOV framework these equations are proven to reconstruct the full spectrum (eigenvalues and eigenstates) of the transfer matrix when one analyze the full class of solutions to (5.36) in a known and model dependent class of functions. The clear interest in the paper $[83]$ is that it proposes an ansatz 10 to associate to the equation of type (5.36) the functional T-Q equations in terms of elliptic polynomials, allowing a more traditional analysis of the eigenvalue problem by the analysis of a system of Bethe equations.

\section{Scalar Products}

The above analysis in SOV allows to get the following scalar product formulae for separate states; one interesting point about them is that they are mainly automatically derived and universal in this framework.

Theorem 6.1. Let $\langle u|$ and $|v\rangle$ be arbitrary states with the following separate forms:

$$
\begin{aligned}
& \langle u|=\sum_{h_{1}, \ldots, h_{\mathrm{N}}=0}^{1} \prod_{a=1}^{\mathrm{N}} u_{a}\left(\zeta_{a}^{\left(h_{a}\right)}\right) \prod_{1 \leq b<a \leq \mathrm{N}}\left(\eta_{a}^{\left(h_{a}\right)}-\eta_{b}^{\left(h_{b}\right)}\right)\left\langle\beta, h_{1}, \ldots, h_{\mathrm{N}}\right|, \\
& |v\rangle=\sum_{h_{1}, \ldots, h_{\mathrm{N}}=0}^{1} \prod_{a=1}^{\mathrm{N}} v_{a}\left(\zeta_{a}^{\left(h_{a}\right)}\right) \prod_{1 \leq b<a \leq \mathrm{N}}\left(\eta_{a}^{\left(h_{a}\right)}-\eta_{b}^{\left(h_{b}\right)}\right)\left|\beta+2, h_{1}, \ldots, h_{\mathrm{N}}\right\rangle,
\end{aligned}
$$

\footnotetext{
${ }^{10}$ An analysis of the open problem of completeness of such type of ansatz has been addressed recently in [85] for the case of the inhomogeneous XXX spin chains.
} 
in the $\mathcal{B}$-pseudo-eigenbasis, then the action of $\langle u|$ on $|v\rangle$ reads:

$$
\langle u \mid v\rangle=\operatorname{det}_{\mathrm{N}}\left\|\mathcal{M}_{a, b}^{(u, v)}\right\| \text { with } \mathcal{M}_{a, b}^{(u, v)} \equiv \sum_{h=0}^{1} u_{a}\left(\zeta_{a}^{(h)}\right) v_{a}\left(\zeta_{a}^{(h)}\right)\left(\eta_{a}^{(h)}\right)^{(b-1)} .
$$

The above formula holds in particular in case the left and right states are transfer matrix eigenstates.

Proof. Formula (4.90) and the definitions of the states $\langle u|$ and $|v\rangle$ imply:

$$
\langle u \mid v\rangle=\sum_{h_{1}, \ldots, h_{\mathrm{N}}=0}^{1} V\left(\eta_{1}^{\left(h_{1}\right)}, \ldots, \eta_{\mathrm{N}}^{\left(h_{\mathrm{N}}\right)}\right) \prod_{a=1}^{\mathrm{N}} u_{a}\left(\zeta_{a}^{\left(h_{a}\right)}\right) v_{a}\left(\zeta_{a}^{\left(h_{a}\right)}\right),
$$

where $V\left(x_{1}, \ldots, x_{\mathrm{N}}\right) \equiv \prod_{1 \leq b<a \leq \mathrm{N}}\left(x_{a}-x_{b}\right)$ is the Vandermonde determinant, then (6.3) follows from the multilinearity of the determinant.

\section{Conclusion and outlook}

In this paper we have considered representation of the 8-vertex reflection algebra and we have studied the quantum models associated to the most general integrable boundary conditions on the spin- $1 / 2$ quantum chains and developed for them the SOV method obtaining the following results:

- The complete integrability of these quantum models and the complete characterization of their spectrum (transfer matrix eigenvalues and eigenstates) in terms of the set of solutions to an inhomogeneous system of $\mathrm{N}$ quadratic equations in $\mathrm{N}$ unkowns, where $\mathrm{N}$ is the number of sites of the chain.

It is important to remark here that for the most general boundary conditions and values of the coupling constant $\eta$ the previous characterization is not yet proven to be equivalent to a characterization in terms of Bethe equations and this equivalence can be surely proven only imposing some constrains on the boundary parameters or on the coupling constant. In particular in a future paper we will show as in the case $\eta$ an elliptic root of unit we can derive for the most general integrable boundary conditions a Baxter Q-operator and rewrite the SOV spectrum characterization in terms of solutions to a system of Bethe equations.

- The action of left separate states on right separate states are written in terms of one determinant formulae of $\mathrm{N} \times \mathrm{N}$ matrices; these matrices have elements given by sums over the spectrum of quantum separate variables of products of the corresponding left/right separate coefficients.

These results define the required setup to compute matrix elements of local operators on transfer matrix eigenstates. The remarked similarities in the SOV representations of the gauge transformed reflection algebras and the form of the pseudo-measure entering in the SOV spectral decomposition of the identity for both the 8-vertex and 6-vertex case imply the possibility to solve in parallel these two a priori very different dynamical problems. In particular, in a future publication we will address the analysis of the following steps:

I) Reconstruction of local operators in terms of Sklyanin's quantum separate variables.

II) Representation of form factors of local operators on transfer matrix eigenstates in determinant form. 
Let us comment that I) is a fundamental step in the solution of the dynamical problem as it allows to identify the local operators writing them in terms of the global generators of the SOV representation. In fact, this identification has represented a longstanding problem in the S-matrix formulation 11 of the dynamics of infinite volume quantum field theories and the lattice approach seems to give the advantage to make it solvable. Moreover, once it is solved it allows to compute algebraically the actions of local operators on transfer matrix eigenstates and write them as linear combinations of separate states from which the form factors can be computed by using our results on the action of left separate states on right separate states. Let us also point out that the reconstructions derived in the 6-vertex reflection algebra case apply also to the 8-vertex reflection algebra and that being both the gauge transformed 8vertex and 6-vertex reflection algebra generators written as linear combinations of the ungauged ones, the solution of the reconstruction problem for the most general integrable boundary conditions is simply derived once it is solved for the ungauged 6-vertex one following the approach described in [1]. This last observation implies that we are already able to describe the matrix elements of a class of quasi-local operators for the most general reflection algebra representations of both 8-vertex and 6-vertex type; indeed, in order to do so we just need to elaborate the results of this paper, those of [2] and the matrix elements in the ungauged SOV framework derived in [1].

Acknowledgments The authors would like to thank N. Kitanine, J.M. Maillet and V. Terras for discussions. G.N. gratefully acknowledge the YITP Institute of Stony Brook for the freedom left in developing his research programs under the National Science Foundation grants PHY-0969739 and the privilege to have stimulating discussions with B. M. McCoy on subjects related to the 8-vertex models. S.F. is supported by the Burgundy region. G.N. would like to thank the Theoretical Physics Group of the Laboratory of Physics at ENS-Lyon and the Mathematical Physics Group at IMB of the Dijon University for their hospitality under the support of the ANR grant ANR-10-BLAN0120-04-DIADEMS.

\section{References}

[1] G. Niccoli.J. Stat. Mech.: Theory and Exp., P10025, 2012.

[2] S. Faldella, N. Kitanine, G. Niccoli, Complete spectrum and scalar products for the open spin-1/2 XXZ quantum chains with non-diagonal boundary terms, arXiv:1307.3960.

[3] E. K. Sklyanin and L. D. Faddeev, Sov. Phys. Dokl. 23 (1978) 902.

[4] E. K. Sklyanin and L. A. Takhtajan, L. D. Faddeev, Theor. Math. Phys. 40 (1980) 688.

[5] L. A. Takhtajan, L. D. Faddeev, Russ. Math. Surv. 34 : 5 (1979) 11.

[6] E. K. Sklyanin, Dokl. Akad. Nauk SSSR 244 (1979) 1337; Sov. Phys. Dokl. 24 (1979) 107.

[7] P. P. Kulish and E. K. Sklyanin, Phys. Lett. A 70 (1979) 461.

[8] L. D. Faddeev, Sov. Sci. Rev. Math. Cl (1980) 107.

[9] E. K. Sklyanin, J. Sov. Math. 19 (1982) 1546.

[10] L. D. Faddeev, Les Houches lectures of 1982, Elsevier Sci. Publ. 563 (1984).

[11] M. Jimbo, Adv. Series in Math. Phys. 10, Singapore, World Scientific, (1990).

[12] P. P. Kulish and E. K. Sklyanin, Lect. Notes in Phys. 151 (1982) 61.

[13] B. S. Shastry, Lect. Notes in Phys. 242 (1985).

[14] H. B. Thacker, Rev. Mod. Phys. 53 (1982) 253.

[15] A. G. Izergin and V. E. Korepin, Nucl. Phys. B 205 (1982) 401.

[16] I. V. Cherednik, Theor. Math. Phys. 61 (1984) 977.

[17] F. C. Alcaraz, M. N. Barber, M. T. Batchelor, R. J. Baxter and G. R. W. Quispel, J. Phys. A: Math. Gen. 20 (1987) 6397.

\footnotetext{
${ }^{11}$ Let us mention that a large literature has been dedicated to this problem and several results are known [92]-[97] which confirm the characterization [103, 104] of these models as (superrenormalizable) massive perturbations of conformal field theories by relevant local fields from which a classification of their local field content (solutions to the form factor equations [105, 106]) can be developed by using the corresponding ultraviolet conformal field theories.
} 
[18] E. K. Sklyanin, J. Phys. A: Math. Gen. 21 (1988) 2375.

[19] P. P. Kulish and E. K. Sklyanin, J. Phys. A: Math. Gen. 24 (1991) L435; J. Phys. A: Math. Gen. 25 (1992) 5963.

[20] L. Mezincescu and R. Nepomechie, Int. J. Mod. Phys. A 6 (1991) 5231.

[21] S. Ghoshal and A. B. Zamolodchikov, Int. J. Mod. Phys. A 9 (1994) 3841; Int. J. Mod. Phys. A 9 (1994) 4353.

[22] H. Bethe. Z. Physik, 71 (1931) 205.

[23] L. Hulthen, Ark. Mat. Astron. Fys. 26 (1938) 1.

[24] R. Orbach, Phys. Rev. 112 (1958) 309.

[25] L. R. Walker, Phys. Rev. 116 (1959) 1089.

[26] C. N. Yang and C. P. Yang, Phys. Rev. 150 (1966) 321.

[27] C. N. Yang and C. P. Yang, Phys. Rev. 150 (1966) 327.

[28] M. Gaudin, La Fonction d'onde de Bethe, Paris: Masson (1983).

[29] E. H. Lieb and D. C. Mattis, Mathematical Physics in One Dimension, New-York: Academic (1966).

[30] J. Cao, H.-Q. Lin, K.-J. Shi, Y. Wang, Nucl. Phys. B 663 (2003) 487.

[31] W.-L. Yang, Y.-Z. Zhang, J. High Energy Phys. 4 (2007) 044.

[32] R. I. Nepomechie, F. Ravanini, J. Phys. A: Math. Gen.36 (2003) 11391.

[33] B. Derrida, Phys. Rep. 301 (1998) 65.

[34] G. M. Schütz, Phase Transitions and Critical Phenomena, London: Academic 19 (2000).

[35] F. C. Alcaraz, M. Droz, M. Henkel and V. Rittenberg, Ann. Phys. 230 (1994) 250.

[36] Z. Bajnok, J. Stat. Mech. (2006) P06010.

[37] J. de Gier and F. H. L. Essler, Phys. Rev. Lett. 95 (2005) 240601; J. Stat. Mech. (2006) P12011.

[38] P. Baseilhac, K. Koizumi, J. Stat. Mech. (2005) P10005; J. Stat. Mech. (2007) P09006.

[39] P. Baseilhac, A family of tridiagonal pairs and related symmetric functions, arXiv:math-ph/0604035, Nucl. Phys. B 754 (2006) 309; New results in the XXZ open spin chain, arXiv:0712.0452.

[40] R. I. Nepomechie, Bethe Ansatz for the open XXZ chain from functional relations at roots of unity, arXiv:hep-th/0510086

[41] R. Murgan, R. I. Nepomechie, and C. Shi, J. Stat. Mech. (2006) P08006.

[42] Wen-Li Yang, R. I. Nepomechie, Y.-Z. Zhang, Phys.Lett. B 633 (2006) 664.

[43] H. Frahm, A. Seel, T. Wirth, Nucl. Phys. B 802 (2008) 351.

[44] L. Amico, H. Frahm, A. Osterloh and G. A. P. Ribeiro, Nucl. Phys. B 787 (2007) 283.

[45] L. Amico, H. Frahm, A. Osterloh, and T. Wirth, Nucl. Phys. B 839 [FS] (2010) 604.

[46] H. Frahm, J. H. Grelik A. Seel, T. Wirth, J. Phys. A 44 (2011) 015001.

[47] E. K. Sklyanin, Lect. Notes Phys. 226 (1985) 196; J. Sov. Math. 31 (1985) 3417; Quantum inverse scattering method. Selected topics. In: Quantum groups and quantum integrable systems, World Scientific (1992) 63, arXiv:hep-th/9211111v1; Prog. Theor. Phys. Suppl. 118 (1995) 35.

[48] G. Niccoli and J. Teschner, J. Stat. Mech. (2010) P09014.

[49] G. Niccoli, Nucl. Phys. B 835 (2010) 263; JHEP 03 (2011)123.

[50] N. Grosjean and G. Niccoli, J. Stat. Mech. (2012) P10006.

[51] N. Grosjean, J. M. Maillet, G. Niccoli, J. Stat. Mech. (2012) P10006.

[52] G. Niccoli, On the developments of Sklyanin's quantum separation of variables for integrable quantum field theories, invited contribution to the proceedings of the "XVIIth INTERNATIONAL CONGRESS ON MATHEMATICAL PHYSICS ICMP12", Aalborg, Danemark, August 2012, in printing on the ICMP12 Proceedings by World Scientific, arXiv:1301.4924

[53] N. Grosjean, J. M. Maillet, G. Niccoli, On matrix elements of local operators in the $\tau_{2}$-model and the chiral Potts model, to appear.

[54] G. Niccoli, Nucl.Phys. B, 870 (2013) 397.

[55] G. Niccoli, J. Math. Phys. 54 (2013) 053516.

[56] G. Niccoli, J. Phys. A: Math. Theor. 46 075003, 2013.

[57] R. J. Baxter, Ann. Phys. 76 (1973) 1; R. J. Baxter, Ann. Phys. 76 (1973) 25; Ann. Phys. 76 (1973) 48.

[58] G. Felder. Elliptic Quantum Groups. In Xlth International Congress of Mathematical Physics, Paris 1994 (D- Ialgonitzer, ed.), International Press (1995) 211.

[59] D. Levy-Bencheton, G. Niccoli and V. Terras, to appear. 
[60] R. J. Baxter, Exactly Solved Models in Statistical Mechanics, Academic Press, New York U.S.A. (1982).

[61] K. Fabricius and B. M. McCoy, J. Stat. Phys. 111 (2003) 323; Functional equations and fusion matrices for the eight vertex model, Publ. RIMS, Kyoto Univ. 40 (2004) 905; J. Stat. Phys. 120 (2005) 37.

[62] K. Fabricius, J. Phys. A 40 (2007) 4075.

[63] K. Fabricius, and B. M. McCoy, J. Phys. A 40 (2007) 14893; J. Stat. Phys. 134 (2009) 643.

[64] H. Fan, B. -Y. Hou, K. -J. Shi and Z. -X. Yang, Nucl. Phys. B 478 (1996), 723.

[65] R. J. Baxter, J. Statist. Phys. 108 (2002) 1.

[66] G. Filali , N. Kitanine, J. Stat. Mech. (2010) L06001; Spin Chains with Non-Diagonal Boundaries and Trigonometric SOS Model with Reflecting End, SIGMA 7:012,2011, arXiv:1011.0660.

[67] G. Filali, Elliptic dynamical reflection algebra and partition function of SOS model with reflecting end, J. of Geometry and Physics (2011), arXiv:1012.0516.

[68] Wen-Li Yang, Xi Chen, Jun Feng, Kun Hao, Bo-Yu Hou, Kang-Jie Shi, Yao-Zhong Zhang, Nucl. Phys. B 844 (2011), 289-307

[69] Wen-Li Yang, Xi Chen, Jun Feng, Kun Hao, Kang-Jie Shi, Cheng-Yi Sun, Zhan-Ying Yang, Yao-Zhong Zhang, Nucl. Phys. B 847 (2011) 367.

[70] N. A. Slavnov, Theor. Math. Phys. 79 (1989) 502; Zap. Nauchn. Semin. POMI 245 (1997) 270.

[71] N. Kitanine, J. M. Maillet, and V. Terras, Nucl. Phys. B 554 (1999) 647.

[72] J. M. Maillet and V. Terras, Nucl. Phys. B 575 (2000) 627.

[73] N. Kitanine, J. M. Maillet, and V. Terras, Nucl. Phys. B 567 (2000) 544.

[74] N. Kitanine, J. Phys. A: Math. Gen. 34 (2001) 8151.

[75] O. A. Castro-Alvaredo, J. M. Maillet, J. Phys. A 40 (2007) 7451.

[76] N. Kitanine, K. K. Kozlowski, J. M. Maillet, G. Niccoli, N. A. Slavnov, V. Terras , J. Stat. Mech. (2007) P10009.

[77] K. K. Kozlowski, J. Stat.Mech. (2008) P02006.

[78] N. Kitanine, K. Kozlowski, J. M. Maillet, G. Niccoli, N. A. Slavnov, V. Terras, J. Stat. Mech. (2008) P07010.

[79] T. Inami and H. Konno, J. Phys. A 27 (1994), L913.

[80] I.S. Gradshteyn and I.M. Ryzhik; Alan Jeffrey, Daniel Zwillinger, editors. Table of Integrals, Series, and Products, seventh edition. Academic Press (2007).

[81] H. J. de Vega and A. Gonzalez-Ruiz, J. Phys. A 26 (1993), L519.

[82] A. G. Izergin and V. E. Korepin, Dokl. Akad. Nauk SSSR 259 (1981) 76.

[83] J. Cao, W. Yang, K. Shi, and Y. Wang. Off-diagonal Bethe ansatz solutions of the anisotropic spin-1/2 chains with arbitrary boundary fields. arXiv:1307.2023

[84] J. Cao, W. Yang, K. Shi, and Y. Wang. Off-diagonal Bethe ansatz and exact solution a topological spin ring. arXiv:1305.7328, Off-diagonal bethe ansatz solution of the XXX spin-chain with arbitrary boundary conditions. arXiv:1306.1742, Spin-1/2 XYZ model revisit: general solutions via off-diagonal Bethe ansatz. arXiv:1307.0280.

[85] R. Nepomechie, Inhomogeneous T-Q equation for the open XXX chain with general boundary terms: completeness and arbitrary spin, arXiv:1307.5049

[86] F. Smirnov, J. Phys. A: Math. Gen. 31 (1998) 8953.

[87] A. Bytsko, J. Teschner, J. Phys. A39 (2006) 12927.

[88] P. P. Kulish, N. Y. Reshetikhin and E. K. Sklyanin, Lett. Math. Phys. 5 (1981) 393.

[89] A. N. Kirillov and N. Y. Reshetikhin, J. Phys. A: Math Gen. 20 (1987) 1565.

[90] V. V. Bazhanov and N. Y. Reshetikhin, Int. J. Mod. Phys. A 4 (1989) 115.

[91] R. I. Nepomechie, Nucl. Phys. B 622 (2002) 615; J. Stat. Phys. 111 (2003) 1363.

[92] J. L. Cardy and G. Mussardo, Nucl. Phys. B 340 (1990) 387.

[93] A. Koubek, Nucl. Phys. B 435 (1995) 703.

[94] F. Smirnov, Nucl. Phys. B 453 (1995) 807.

[95] O. Babelon, D. Bernard, F. Smirnov, Comm. Math. Phys. 182 (1996) 319; Comm. Math. Phys. 186 (1997) 601.

[96] M. Jimbo, T. Miwa, Y. Takeyama, Counting minimal form factors of the restricted sine-Gordon model, arXiv:math-ph/0303059v6.

[97] G. Delfino and G. Niccoli, Nucl. Phys. B 707 (2005) 381; J. Stat. Mech. (2005) P04004; JHEP 05 (2006) 035; Nucl. Phys. B 799 (2008) 364.

[98] A. B. Zamolodchikov, Pis. Zh. Eksp. Teor. Fiz. 25 (1977) 499; Comm. Math. Phys. 55 (1977) 183. 
[99] A.B. Zamolodchikov, Al. B. Zamolodchikov, Ann. Phys. 120 (1979) 253.

[100] M. Karowski and H. J. Thun, Nucl. Phys. B 130 (1977) 295.

[101] V. Korepin, Comm. Math. Phys. 76 (1980) 165.

[102] G. Mussardo, Phys. Rep. 218 (1992) 215.

[103] A. B. Zamolodchikov, Int. J. Mod. Phys. A 3 (1988) 743; Adv. Stud. Pure Math. 19 (1989) 641.

[104] Al. B. Zamolodchikov, Nucl. Phys. B 348 (1991) 619.

[105] M. Karowski, P. Weisz, Nucl. Phys. B 139 (1978) 455.

[106] F. A. Smirnov, Form Factors in Completely Integrable Models of Quantum Field Theory, World Scientific (1992). 